\title{
ON WITTEN MULTIPLE ZETA-FUNCTIONS ASSOCIATED WITH SEMISIMPLE LIE ALGEBRAS III
}

\author{
YASUSHI KOMORI, KOHJI MATSUMOTO AND HIROFUMI TSUMURA
}

\begin{abstract}
We prove certain general forms of functional relations among Witten multiple zeta-functions in several variables (or zeta-functions of root systems). The structural background of those functional relations is given by the symmetry with respect to Weyl groups. From those relations we can deduce explicit expressions of values of Witten zeta-functions at positive even integers, which is written in terms of generalized Bernoulli numbers of root systems. Furthermore we introduce generating functions of those Bernoulli numbers of root systems, by which we can give an algorithm of calculating Bernoulli numbers of root systems.
\end{abstract}

\section{INTRODUCTION}

Let $\mathfrak{g}$ be a complex semisimple Lie algebra with rank $r$. The Witten zeta-function associated with $\mathfrak{g}$ is defined by

$$
\zeta_{W}(s ; \mathfrak{g})=\sum_{\varphi}(\operatorname{dim} \varphi)^{-s}
$$

where the summation runs over all finite dimensional irreducible representations $\varphi$ of $\mathfrak{g}$.

Let $\mathbb{N}$ be the set of positive integers, $\mathbb{N}_{0}=\mathbb{N} \cup\{0\}, \mathbb{Z}$ the ring of rational integers, $\mathbb{Q}$ the rational number field, $\mathbb{R}$ the real number field, $\mathbb{C}$ the complex number field, respectively. Witten's motivation [35] of introducing the above zeta-function is to express the volumes of certain moduli spaces in terms of special values of (1.1). This expression is called Witten's volume formula, which especially implies that

$$
\zeta_{W}(2 k ; \mathfrak{g})=C_{W}(2 k, \mathfrak{g}) \pi^{2 k n}
$$

for any $k \in \mathbb{N}$, where $n$ is the number of all positive roots and $C_{W}(2 k, \mathfrak{g}) \in \mathbb{Q}$ (Witten [35], Zagier [36]).

When $\mathfrak{g}=\mathfrak{s l}(2)$, the corresponding Witten zeta-function is nothing but the Riemann zeta-function $\zeta(s)$. It is classically known that

$$
\zeta(2 k)=\frac{-1}{2} \frac{(2 \pi \sqrt{-1})^{2 k}}{(2 k) !} B_{2 k} \quad(k \in \mathbb{N}),
$$

where $B_{2 k}$ is the $2 k$-th Bernoulli number. Formula (1.2) is a generalization of (1.3), but the values $C_{W}(2 k, \mathfrak{g})$ are not explicitly determined in the work of Witten and of Zagier. In the present paper we introduce a root-system theoretic generalization of Bernoulli numbers and periodic Bernoulli functions, and express $C_{W}(2 k, \mathfrak{g})$ explicitly in terms of

Y. Komori: Graduate School of Mathematics, Nagoya University, Chikusa-ku, Nagoya 464-8602 Japan, email: komori@math.nagoya-u.ac.jp

K. Matsumoto: Graduate School of Mathematics, Nagoya University, Chikusa-ku, Nagoya 464-8602 Japan, email: kohjimat@math.nagoya-u.ac.jp

H. Tsumura: Department of Mathematics and Information Sciences, Tokyo Metropolitan University, 1-1, Minami-Ohsawa, Hachioji, Tokyo 192-0397 Japan, email: tsumura@tmu.ac.jp. 
those generalized Bernoulli periodic functions $P(\mathbf{k}, \mathbf{y} ; \Delta)$ (defined in Section 4). This result will be given in Theorem 4.6. Note that Szenes [28,29] also studied generalizations of Bernoulli polynomials from the viewpoint of the theory of arrangement of hyperplanes, which include $P(\mathbf{k}, \mathbf{y} ; \Delta)$ mentioned above. However, our root-system theoretic approach enables us to show that our $P(\mathbf{k}, \mathbf{y} ; \Delta)$ and its generating function $F(\mathbf{k}, \mathbf{y} ; \Delta)$ are quite natural extensions of the classical ones (see Theorems proved in Section 6).

Our explicit expression of $C_{W}(2 k, \mathfrak{g})$ is obtained as special cases of a general form of functional relations, which is another main result of the present paper. To explain this, we first introduce the multi-variable version of Witten zeta-functions.

Let $\Delta$ be the set of all roots of $\mathfrak{g}, \Delta_{+}$the set of all positive roots of $\mathfrak{g}, \Psi=\left\{\alpha_{1}, \ldots, \alpha_{r}\right\}$ the fundamental system of $\Delta, \alpha_{j}^{\vee}$ the coroot associated with $\alpha_{j}(1 \leq j \leq r)$. Let $\lambda_{1}, \ldots, \lambda_{r}$ be the fundamental weights satisfying $\left\langle\alpha_{i}^{\vee}, \lambda_{j}\right\rangle=\lambda_{j}\left(\alpha_{i}^{\vee}\right)=\delta_{i j}$ (Kronecker's delta). A more explicit form of $\zeta_{W}(s ; \mathfrak{g})$ can be written down in terms of roots and weights by using Weyl's dimension formula (see (1.4) of [12]). Inspired by that form, we introduced in [12] the multi-variable version of Witten zeta-function

$$
\zeta_{r}(\mathbf{s} ; \mathfrak{g})=\sum_{m_{1}=1}^{\infty} \cdots \sum_{m_{r}=1}^{\infty} \prod_{\alpha \in \Delta_{+}}\left\langle\alpha^{\vee}, m_{1} \lambda_{1}+\cdots+m_{r} \lambda_{r}\right\rangle^{-s_{\alpha}},
$$

where $\mathbf{s}=\left(s_{\alpha}\right)_{\alpha \in \Delta_{+}} \in \mathbb{C}^{n}$. In the case that $\mathfrak{g}$ is of type $X_{r}$, we call (1.4) the zeta-function of the root system of type $X_{r}$, and also denote it by $\zeta_{r}\left(\mathbf{s} ; X_{r}\right)$, where $X=A, B, C, D, E, F, G$. Note that from (1.5) and (1.7) in [12], we have

$$
\zeta_{W}(s ; \mathfrak{g})=K(\mathfrak{g})^{s} \zeta_{r}(s, \ldots, s ; \mathfrak{g}),
$$

where

$$
K(\mathfrak{g})=\prod_{\alpha \in \Delta_{+}}\left\langle\alpha^{\vee}, \lambda_{1}+\cdots+\lambda_{r}\right\rangle .
$$

More generally, in [12], we introduced multiple zeta-functions associated with sets of roots. The main body of [12] is devoted to the study of recursive structures in the family of those zeta-functions, which can be described in terms of Dynkin diagrams of underlying root systems.

Now we discuss what are functional relations.

The Euler-Zagier $r$-fold sum is defined by the multiple series

$$
\begin{aligned}
\zeta_{r}\left(s_{1}, \ldots, s_{r}\right)=\sum_{m_{1}=1}^{\infty} & \cdots \sum_{m_{r}=1}^{\infty} m_{1}^{-s_{1}}\left(m_{1}+m_{2}\right)^{-s_{2}} \times \\
& \times \cdots \times\left(m_{1}+\cdots+m_{r}\right)^{-s_{r}} .
\end{aligned}
$$

The harmonic product formula

$$
\zeta\left(s_{1}\right) \zeta\left(s_{2}\right)=\zeta\left(s_{1}+s_{2}\right)+\zeta_{2}\left(s_{1}, s_{2}\right)+\zeta_{2}\left(s_{2}, s_{1}\right)
$$

due to L. Euler (where $\zeta(s)$ denotes the Riemann zeta-function), and its $r$-ple analogue, are classically known (cf. Bradley [3]). However, no other functional relations among multiple zeta-functions has been discovered for a long time.

Let

$$
\zeta_{M T, 2}\left(s_{1}, s_{2}, s_{3}\right)=\sum_{m=1}^{\infty} \sum_{n=1}^{\infty} m^{-s_{1}} n^{-s_{2}}(m+n)^{-s_{3}} .
$$


This series is called Tornheim's harmonic double sum or the Mordell-Tornheim double zeta-function, after the work of Tornheim [30] and Mordell [24] in 1950s. But it is to be noted that this sum actually coincides with the Witten zeta-function (1.4) for $\mathfrak{g}=\mathfrak{s l}(3)$, that is, the simple Lie algebra of type $A_{2}$. Recently the third-named author [33] proved that there are certain functional relations among $\zeta_{M T, 2}\left(s_{1}, s_{2}, s_{3}\right)=\zeta_{2}\left(s_{1}, s_{2}, s_{3} ; A_{2}\right)$ and the Riemann zeta-function. Moreover he obtained the same type of functional relations for various relatives of $\zeta_{M T, 2}\left(s_{1}, s_{2}, s_{3}\right)$ (see [31,32]). The method in these papers can be called the " $u$-method", because an auxiliary parameter $u>1$ was introduced to ensure the absolute convergence in the argument.

In [21], by the same " $u$-method", the second and the third-named authors proved certain functional relations among $\zeta_{3}\left(\mathbf{s} ; A_{3}\right), \zeta_{2}\left(\mathbf{s} ; A_{2}\right)$ and the Riemann zeta-function. The papers $[19,20,22,23]$ are also devoted to the study of some new functional relations for certain (mainly) double and triple zeta-functions and their relatives.

The above papers give many examples of functional relations. Therefore, now, it is time appropriate to investigate whether there is some structural reason underlying those functional relations, or not. The first hint on this question was supplied by Nakamura's paper [25], in which he presented a new simple proof of the result of the third-named author [33]. (Nakamura's method has then been applied in [19,20].)

It can be observed from Nakamura's proof that the functional relations proved in $[25,33]$ are connected with the symmetricity with respect to the symmetric group $\mathfrak{S}_{3}$, which is the Weyl group associated with the Lie algebra of type $A_{2}$. This suggests the possibility of formulating general functional relations in terms of Weyl groups of Lie algebras.

One of the main purposes of the present paper is to show that such general forms of functional relations can indeed be proved. In Section 2, we prepare some notation and preliminary results about root systems, Weyl groups and convex polytopes, which play essential roles in the study of the structural background of functional relations. We will give general forms of functional relations in Sections 3 and 4 . The most general form of functional relations is Theorem [3.1, which is specialized to the case of "Weyl group symmetric" linear combinations $S(\mathbf{s}, \mathbf{y} ; I ; \Delta)$ (defined by (4.6)) of zeta-functions of root systems with exponential factors in Theorem 4.3 and Theorem 4.4. A naïve form of Theorem 4.4 has been announced in Section 3 of [9], but we consider the generalized form with exponential factors because this form can be applied to evaluations of $L$-functions of root systems (see [14] for the details.)

The theorems mentioned above give expressions of linear combinations of zeta-functions in terms of certain multiple integrals involving Lerch zeta-functions. Since the values of Lerch zeta-functions at positive integers $(\geq 2)$ can be written as Bernoulli polynomials, we can show more explicit forms of those multiple integrals in some special cases. We will carry out this procedure by using generating functions (Theorem 3.5).

In particular, we find that the value $S(\mathbf{s}, \mathbf{y} ; \Delta)=S(\mathbf{s}, \mathbf{y} ; \emptyset ; \Delta)$ at $\mathbf{s}=\mathbf{k}=\left(k_{\alpha}\right)$, where all $k_{\alpha}$ 's are positive integers $(\geq 2)$, is essentially a generalization $P(\mathbf{k}, \mathbf{y} ; \Delta)$ of periodic Bernoulli functions. The generating function $F(\mathbf{t}, \mathbf{y} ; \Delta)$ of $P(\mathbf{k}, \mathbf{y} ; \Delta)$ will be evaluated in Theorem 4.5. Consequently, we can prove a generalization of Witten's volume formula (1.2) (Theorem 4.6).

In Section 5, we will show the Weyl group symmetry of $S(\mathbf{s}, \mathbf{y} ; \Delta), F(\mathbf{t}, \mathbf{y} ; \Delta)$ and $P(\mathbf{k}, \mathbf{y} ; \Delta)$. For our purpose it is not sufficient to consider the usual Weyl group only, and hence we will introduce the extended affine Weyl group $\widehat{W}$, and will prove the symmetricity with respect to $\widehat{W}$ (Theorems 5.1, 5.5 and 5.8). These results ensure that the existence of 
functional relations is indeed based on the symmetry with respect to $\widehat{W}$. It is to be noted that Weyl groups already played a role in Zagier's sketch [36] of the proof of (1.2).

In Section 6 we will prove that $P(\mathbf{k}, \mathbf{y} ; \Delta)$ can be continued to polynomials in $\mathbf{y}$ (Theorem 6.2). This may be regarded as a (root-system theoretic) generalization of Bernoulli polynomials. Since $P(\mathbf{k}, \mathbf{y} ; \Delta)$ is essentially the same as $S(\mathbf{k}, \mathbf{y} ; \Delta)$, this gives explicit relations among special values of zeta-functions of root systems. Moreover in the same theorem we will give the continuation of $F(\mathbf{t}, \mathbf{y} ; \Delta)$.

As examples, in Section 7 we will calculate $P$ and $F$ explicitly in the cases of $A_{1}, A_{2}$, $A_{3}$ and $C_{2}\left(\simeq B_{2}\right)$. In particular, from the explicit expansion of generating functions $F$, we will determine the value of $C_{W}\left(2, A_{2}\right), C_{W}\left(2, A_{3}\right)$ and $C_{W}\left(2, C_{2}\right)$ in (1.2). The cases of $C_{W}\left(2, A_{2}\right), C_{W}\left(2, A_{3}\right)$ are included in the results of Mordell [24], Subbarao et al. [26] and Gunnells-Sczech [4], while the determination of the value of $C_{W}\left(2, C_{2}\right)$ seems to be a new result. By the same method it is possible to obtain the explicit values of $C_{W}\left(2 k, A_{2}\right)$, $C_{W}\left(2 k, A_{3}\right), C_{W}\left(2 k, C_{2}\right)$ for any $k \in \mathbb{N}$. More generally, we can deduce explicit value relations and special values of zeta-functions of any simple algebra $\mathfrak{g}$ by the same argument, at least in principle, though the actual procedure will become quite complicated when the rank of $\mathfrak{g}$ becomes higher. We also provide an example of a functional relation in the $A_{2}$ case.

Some parts of the contents of [12] and the present paper have been already announced briefly in $[9-11]$.

\section{Root systems, Weyl groups And CONVEx POlytopes}

In this preparatory section, we first fix notation and summarize basic facts about root systems and Weyl groups. See $[2,6-8]$ for the details. Let $V$ be an $r$-dimensional real vector space equipped with an inner product $\langle\cdot, \cdot\rangle$. We denote the norm of $v \in V$ by $\|v\|=\langle v, v\rangle^{1 / 2}$. The dual space $V^{*}$ is identified with $V$ via the inner product of $V$. Let $\Delta$ be a finite reduced root system in $V$ and $\Psi=\left\{\alpha_{1}, \ldots, \alpha_{r}\right\}$ its fundamental system. Let $\Delta_{+}$and $\Delta_{-}$be the set of all positive roots and negative roots respectively. Then we have a decomposition of the root system $\Delta=\Delta_{+} \amalg \Delta_{-}$. Let $Q^{\vee}$ be the coroot lattice, $P$ the weight lattice, $P_{+}$the set of integral dominant weights and $P_{++}$the set of integral strongly dominant weights respectively defined by

$$
Q^{\vee}=\bigoplus_{i=1}^{r} \mathbb{Z} \alpha_{i}^{\vee}, \quad P=\bigoplus_{i=1}^{r} \mathbb{Z} \lambda_{i}, \quad P_{+}=\bigoplus_{i=1}^{r} \mathbb{N}_{0} \lambda_{i}, \quad P_{++}=\bigoplus_{i=1}^{r} \mathbb{N} \lambda_{i},
$$

where the fundamental weights $\left\{\lambda_{j}\right\}_{j=1}^{r}$ are a basis dual to $\Psi^{\vee}$ satisfying $\left\langle\alpha_{i}^{\vee}, \lambda_{j}\right\rangle=\delta_{i j}$. Let

$$
\rho=\frac{1}{2} \sum_{\alpha \in \Delta_{+}} \alpha=\sum_{j=1}^{r} \lambda_{j}
$$

be the lowest strongly dominant weight. Then $P_{++}=P_{+}+\rho$.

We define the reflection $\sigma_{\alpha}$ with respect to a root $\alpha \in \Delta$ as

$$
\sigma_{\alpha}: V \rightarrow V, \quad \sigma_{\alpha}: v \mapsto v-\left\langle\alpha^{\vee}, v\right\rangle \alpha
$$

and for a subset $A \subset \Delta$, let $W(A)$ be the group generated by reflections $\sigma_{\alpha}$ for $\alpha \in A$. Let $W=W(\Delta)$ be the Weyl group. Then $\sigma_{j}=\sigma_{\alpha_{j}}(1 \leq j \leq r)$ generates $W$. Namely we have $W=W(\Psi)$. Any two fundamental systems $\Psi, \Psi^{\prime}$ are conjugate under $W$. 
We denote the fundamental domain called the fundamental Weyl chamber by

$$
C=\left\{v \in V \mid\left\langle\Psi^{\vee}, v\right\rangle \geq 0\right\},
$$

where $\left\langle\Psi^{\vee}, v\right\rangle$ means any of $\left\langle\alpha^{\vee}, v\right\rangle$ for $\alpha^{\vee} \in \Psi^{\vee}$. Then $W$ acts on the set of Weyl chambers $W C=\{w C \mid w \in W\}$ simply transitively. Moreover if $w x=y$ for $x, y \in C$, then $x=y$ holds. The stabilizer $W_{x}$ of a point $x \in V$ is generated by the reflections which stabilize $x$. We see that $P_{+}=P \cap C$.

Let $\operatorname{Aut}(\Delta)$ be the subgroup of all the automorphisms GL $(V)$ which stabilizes $\Delta$ (see $[6$, $\S 12.2])$. Then the Weyl group $W$ is a normal subgroup of $\operatorname{Aut}(\Delta)$ and there exists a subgroup $\Omega \subset \operatorname{Aut}(\Delta)$ such that

$$
\operatorname{Aut}(\Delta)=\Omega \ltimes W .
$$

The subgroup $\Omega$ is isomorphic to the group $\operatorname{Aut}(\Gamma)$ of automorphisms of the Dynkin diagram $\Gamma$. The group $\operatorname{Aut}(\Delta)$ is called the extended Weyl group. For $w \in \operatorname{Aut}(\Delta)$, we set

$$
\Delta_{w}=\Delta_{+} \cap w^{-1} \Delta_{-}
$$

and the length function $\ell(w)=\left|\Delta_{w}\right|$ (see $[7, \S 1.6]$ ). The subgroup $\Omega$ is characterized as $w \in \Omega$ if and only if $\ell(w)=0$. Note that $w \Delta_{w}=\Delta_{-} \cap w \Delta_{+}=-\Delta_{w^{-1}}$ and $\ell(w)=\ell\left(w^{-1}\right)$.

For $u \in V$, let $\tau(u)$ be the translation by $u$, that is,

$$
\tau(u): V \rightarrow V, \quad \tau(u): v \mapsto v+u .
$$

Since $\operatorname{Aut}(\Delta)$ stabilizes the coroot lattice $Q^{\vee}$, we can define

$$
\widehat{W}=\operatorname{Aut}(\Delta) \ltimes \tau\left(Q^{\vee}\right) .
$$

Then $\widehat{W}=(\Omega \ltimes W) \ltimes \tau\left(Q^{\vee}\right) \simeq \Omega \ltimes\left(W \ltimes \tau\left(Q^{\vee}\right)\right)$. We call $\widehat{W}$ the extended affine Weyl group in this paper (see $[2,8]$ for the details of affine Weyl groups). It should be noted that there are some other groups which are, in some references, called the extended affine Weyl group.

Let $\widehat{V}=V \times \mathbb{R}$ and $\delta=(0,1) \in \widehat{V}$. We embed $V$ in $\widehat{V}$ and we have $\widehat{V}=V \oplus \mathbb{R} \delta$. For $\gamma=\eta+c \delta \in \widehat{V}$ with $\eta \in V$ and $c \in \mathbb{R}$, we associate an affine linear functional on $V$ as $\gamma(v)=\langle\eta, v\rangle+c$. Let $\widehat{Q}^{\vee}$ be the affine coroot lattice defined by

$$
\widehat{Q}^{\vee}=Q^{\vee} \oplus \mathbb{Z} \delta
$$

(see [8]). For a set $X$, let $\mathfrak{F}(X)$ be the set of all functions $f: X \rightarrow \mathbb{C}$. For a function $f \in \mathfrak{F}(P)$, we define a subset

$$
H_{f}=\{\lambda \in P \mid f(\lambda)=0\}
$$

and for a subset $A$ of $\mathfrak{F}(P)$, define $H_{A}=\bigcup_{f \in A} H_{f}$. One sees that an action of $W$ is induced on $\mathfrak{F}(P)$ as $(w f)(\lambda)=f\left(w^{-1} \lambda\right)$. Note that $V \subset \widehat{V} \subset \mathfrak{F}(P)$, where the second inclusion is given by the associated functional mentioned above.

Let $I \subset\{1, \ldots, r\}$ and $\Psi_{I}=\left\{\alpha_{i} \mid i \in I\right\} \subset \Psi$. Let $V_{I}$ be the linear subspace spanned by $\Psi_{I}$. Then $\Delta_{I}=\Delta \cap V_{I}$ is a root system in $V_{I}$ whose fundamental system is $\Psi_{I}$. For the root system $\Delta_{I}$, we denote the corresponding coroot lattice (resp. weight lattice etc.) by $Q_{I}^{\vee}=\bigoplus_{i \in I} \mathbb{Z} \alpha_{i}^{\vee}$ (resp. $P_{I}=\bigoplus_{i \in I} \mathbb{Z} \lambda_{i}$ etc.). We define

$$
C_{I}=\left\{v \in C \mid\left\langle\Psi_{I^{c}}^{\vee}, v\right\rangle=0, \quad\left\langle\Psi_{I}^{\vee}, v\right\rangle>0\right\},
$$


where $I^{c}$ is the complement of $I$. Then the dimension of the linear span of $C_{I}$ is $|I|$ and we have a disjoint union

$$
C=\coprod_{I \subset\{1, \ldots, r\}} C_{I}
$$

and the collection of all sets $w C_{I}$ for $w \in W$ and $I \subset\{1, \ldots, r\}$ is called the Coxeter complex (see $[7, \S 1.15]$; it should be noted that we use a little different notation), which partitions $V$ and we have a decomposition

$$
P_{+}=\coprod_{I \subset\{1, \ldots, r\}} P_{I++},
$$

where

$$
P_{I++}=P_{+} \cap C_{I}
$$

In particular, $P_{\emptyset++}=\{0\}$ and $P_{\{1, \ldots, r\}++}=P_{++}$.

The natural embedding $\iota: Q_{I}^{\vee} \rightarrow Q^{\vee}$ induces the projection $\iota^{*}: P \rightarrow P_{I}$. Namely for $\lambda \in P, \iota^{*}(\lambda)$ is defined as a unique element of $P_{I}$ satisfying $\langle\iota(q), \lambda\rangle=\left\langle q, \iota^{*}(\lambda)\right\rangle$ for all $q \in Q_{I}^{\vee}$. Let

$$
W^{I}=\left\{w \in W \mid \Delta_{I+}^{\vee} \subset w \Delta_{+}^{\vee}\right\} .
$$

Then we have the following key lemmas to functional relations among zeta-functions. Note that the statements hold trivially in the case $I=\emptyset$ and hence we deal with $I \neq \emptyset$ in their proofs.

Lemma 2.1. The subset $W^{I}$ coincides with the minimal (right) coset representatives $\left\{w \in W \mid \ell\left(\sigma_{i} w\right)>\ell(w)\right.$ for all $\left.i \in I\right\}$ of the parabolic subgroup $W\left(\Delta_{I}\right)$ (see [7, §1.10]). Therefore $\left|W^{I}\right|=\left(W(\Delta): W\left(\Delta_{I}\right)\right)$.

Proof. Let $w \in W^{I}$. Then $\Delta_{I+}^{\vee} \subset w \Delta_{+}^{\vee}$, which implies $\Delta_{I+}^{\vee} \cap w \Delta_{-}^{\vee}=\emptyset$. In particular, $\alpha_{i}^{\vee} \notin w \Delta_{-}^{\vee}$ for $i \in I$, which yields $\Delta_{+}^{\vee} \cap w \Delta_{-}^{\vee}=\left(\Delta_{+}^{\vee} \backslash\left\{\alpha_{i}^{\vee}\right\}\right) \cap w \Delta_{-}^{\vee}$. Therefore

(2.16) $\sigma_{i}\left(\Delta_{+}^{\vee} \cap w \Delta_{-}^{\vee}\right)=\sigma_{i}\left(\left(\Delta_{+}^{\vee} \backslash\left\{\alpha_{i}^{\vee}\right\}\right) \cap w \Delta_{-}^{\vee}\right)=\left(\Delta_{+}^{\vee} \backslash\left\{\alpha_{i}^{\vee}\right\}\right) \cap \sigma_{i} w \Delta_{-}^{\vee} \subset \Delta_{+}^{\vee} \cap \sigma_{i} w \Delta_{-}^{\vee}$

and $\ell\left(\sigma_{i} w\right) \geq \ell(w)$. Since $\ell\left(\sigma_{i} w\right)=\ell(w) \pm 1$, we have $\ell\left(\sigma_{i} w\right)=\ell(w)+1$ and $w$ is a minimal coset representative.

Assume that $w \in W$ satisfies $\ell\left(\sigma_{i} w\right)>\ell(w)$ for all $i \in I$. Then we have

$$
\sigma_{i}\left(\Delta_{+}^{\vee} \cap w \Delta_{-}^{\vee}\right)=\left(\left(\Delta_{+}^{\vee} \backslash\left\{\alpha_{i}^{\vee}\right\}\right) \cap \sigma_{i} w \Delta_{-}^{\vee}\right) \cup\left(\left\{-\alpha_{i}\right\} \cap \sigma_{i} w \Delta_{-}^{\vee}\right) .
$$

Since $\left|\sigma_{i}\left(\Delta_{+}^{\vee} \cap w \Delta_{-}^{\vee}\right)\right|=\ell(w)$ and $\left|\left(\Delta_{+}^{\vee} \backslash\left\{\alpha_{i}^{\vee}\right\}\right) \cap \sigma_{i} w \Delta_{-}^{\vee}\right| \geq \ell\left(\sigma_{i} w\right)-1=\ell(w)$, we have $\left|\left\{-\alpha_{i}\right\} \cap \sigma_{i} w \Delta_{-}^{\vee}\right|=0$. It implies that no element of $\Delta_{+}^{\vee} \cap w \Delta_{-}^{\vee}$ is sent to $\Delta_{-}^{\vee}$ by $\sigma_{i}$ for $i \in I$, and hence $\Psi_{I}^{\vee} \cap w \Delta_{-}^{\vee}=\emptyset$. Since $0 \notin\left\langle\Delta^{\vee}, \rho\right\rangle$ and $\alpha^{\vee} \in w \Delta_{-}^{\vee}$ if and only if $\left\langle\alpha^{\vee}, w \rho\right\rangle<0$, we have $\left\langle\Psi_{I}^{\vee}, w \rho\right\rangle>0$ and hence $\left\langle\Delta_{I+}^{\vee}, w \rho\right\rangle>0$. It follows that $\Delta_{I+}^{\vee} \cap w \Delta_{-}^{\vee}=\emptyset$ and $w \in W^{I}$.

Lemma 2.2.

$$
\iota^{*-1}\left(P_{I+}\right)=P_{I+} \oplus P_{I^{c}}=\bigcup_{w \in W^{I}} w P_{+} .
$$

Proof. The first equality is clear. Prove the second equality.

Assume $w \in W^{I}$. Then for $\lambda \in P_{+}$, we have $\left\langle\Delta_{I+}^{\vee}, w \lambda\right\rangle=\left\langle w^{-1} \Delta_{I+}^{\vee}, \lambda\right\rangle \subset\left\langle\Delta_{+}^{\vee}, \lambda\right\rangle \geq 0$. Hence $w P_{+} \subset \iota^{*-1}\left(P_{I+}\right)$. 
Conversely, assume $\lambda \in \iota^{*-1}\left(P_{I+}\right)$. Since $\left|\Delta^{\vee}\right|<\infty$, it is possible to fix a sufficiently small constant $c>0$ such that $0<\left|\left\langle\Delta^{\vee}, c \rho\right\rangle\right|<1$. Then we see that $\lambda+c \rho$ is regular (see $[6, \S 10.1]$ ), i.e., $0 \notin\left\langle\Delta^{\vee}, \lambda+c \rho\right\rangle$ and the signs of $\left\langle\alpha^{\vee}, \lambda\right\rangle$ and $\left\langle\alpha^{\vee}, \lambda+c \rho\right\rangle$ coincide if $\left\langle\alpha^{\vee}, \lambda\right\rangle \neq 0$, because $\left\langle\Delta^{\vee}, \lambda\right\rangle \subset \mathbb{Z}$. Let $\tilde{\Delta}_{+}^{\vee}=\left\{\alpha^{\vee} \in \Delta^{\vee} \mid\left\langle\alpha^{\vee}, \lambda+c \rho\right\rangle>0\right\}$. Then $\tilde{\Delta}_{+}^{\vee}$ is a positive system and hence there exists an element $w \in W$ such that $\tilde{\Delta}_{+}^{\vee}=w \Delta_{+}^{\vee}$. Since $\lambda \in \iota^{*-1}\left(P_{I+}\right)$, we have $\Delta_{I+}^{\vee} \subset \tilde{\Delta}_{+}^{\vee}$. Hence $\Delta_{I+}^{\vee} \subset w \Delta_{+}^{\vee}$, that is, $w \in W^{I}$. Moreover $\left\langle\tilde{\Delta}_{+}^{\vee}, \lambda+c \rho\right\rangle>0$ implies $\left\langle\Delta_{+}^{\vee}, w^{-1}(\lambda+c \rho)\right\rangle>0$ and $\left\langle\Delta_{+}^{\vee}, w^{-1} \lambda\right\rangle \geq 0$ again due to the integrality. Therefore $\lambda \in w P_{+}$.

Lemma 2.3. For $\lambda \in \iota^{*-1}\left(P_{I_{+}}\right)$, an element $w \in W^{I}$ satisfying $\lambda \in w P_{+}$(whose existence is assured by Lemma 2.2) is unique if and only if $\lambda \notin H_{\Delta \vee} \backslash \Delta_{I}^{\vee}$.

Proof. Assume $\alpha^{\vee} \in \Delta^{\vee} \backslash \Delta_{I}^{\vee}$ and $\lambda \in \iota^{*-1}\left(P_{I+}\right) \cap H_{\alpha^{\vee}}$. Let $w \in W^{I}$ satisfy $\lambda \in w P_{+}$. Then $\sigma_{\alpha} \lambda=\lambda \in w P_{+}$and hence $w^{-1} \lambda=\sigma_{w^{-1} \alpha} w^{-1} \lambda \in P_{+}$, which further implies $w^{-1} \alpha^{\vee} \in \Delta^{\prime \vee}$, where $\Delta^{\prime \vee}$ is a coroot system orthogonal to $w^{-1} \lambda$ whose fundamental system is given by $\Psi^{\prime \vee}=\left\{\alpha_{i}^{\vee} \in \Psi^{\vee} \mid\left\langle\alpha_{i}^{\vee}, w^{-1} \lambda\right\rangle=0\right\}$ (see [7, §1.12]). If $\Psi^{\prime \vee} \subset w^{-1} \Delta_{I}^{\vee}$, then $W\left(\Psi^{\prime \vee}\right) \Psi^{\prime \vee}=\Delta^{\prime \vee} \subset w^{-1} \Delta_{I}^{\vee}$, and hence $w^{-1} \alpha^{\vee} \in w^{-1} \Delta_{I}^{\vee}$, which contradicts to the assumption $\alpha^{\vee} \notin \Delta_{I}^{\vee}$. Therefore there exists a fundamental coroot $\alpha_{i}^{\vee} \in \Psi^{\prime \vee} \backslash w^{-1} \Delta_{I}^{\vee}$, which satisfies $\sigma_{i} w^{-1} \lambda=w^{-1} \lambda \in P_{+}$by construction. Since $w \in W^{I}$, we have $w^{-1} \Delta_{I+}^{\vee} \subset$ $\Delta_{+}^{\vee} \backslash\left\{\alpha_{i}^{\vee}\right\}$. Hence $\sigma_{i} w^{-1} \Delta_{I+}^{\vee} \subset \Delta_{+}^{\vee}$, because $\sigma_{i}\left(\Delta_{+}^{\vee} \backslash\left\{\alpha_{i}^{\vee}\right\}\right) \subset \Delta_{+}^{\vee}$. Then putting $w^{\prime}=w \sigma_{i}$, we have $W^{I} \ni w^{\prime} \neq w$ such that $\lambda \in w P_{+} \cap w^{\prime} P_{+}$.

Conversely, assume that there exist $w, w^{\prime} \in W^{I}$ such that $w \neq w^{\prime}$ and $\lambda \in w P_{+} \cap w^{\prime} P_{+}$. This implies that $w^{-1} \lambda=w^{-1} \lambda$ is on a wall of $C$ and hence $\lambda \in H_{\Delta} \vee$. Let $\Delta^{\prime \prime \vee}=\left\{\alpha^{\vee} \in\right.$ $\left.\Delta^{\vee} \mid \lambda \in H_{\alpha^{\vee}}\right\}$ be a coroot system orthogonal to $\lambda$ so that $\lambda \in H_{\Delta^{\prime \prime}}$. Assume $\Delta^{\prime \prime \vee} \subset \Delta_{I}^{\vee}$. Then by $\lambda=w w^{-1} \lambda$, we have $w w^{\prime-1} \in W_{\lambda}$ and hence $w w^{\prime-1} \in W\left(\Delta_{I}\right)$, because $W_{\lambda}=$ $W\left(\Delta^{\prime \prime \vee}\right) \subset W\left(\Delta_{I}^{\vee}\right)$ by the assumption. Since id $\neq w w^{\prime-1} \in W\left(\Delta_{I}\right)$, there exists a coroot $\alpha^{\vee} \in \Delta_{I+}^{\vee}$ such that $\beta^{\vee}=w w^{\prime-1} \alpha^{\vee} \in \Delta_{I-}^{\vee}$. Then, since $w^{-1}\left(w w^{\prime-1}\right) \Delta_{I+}^{\vee} \subset \Delta_{+}^{\vee}$ and $w^{-1} \Delta_{I+}^{\vee} \subset \Delta_{+}^{\vee}$, we have $w^{-1} \beta^{\vee} \in \Delta_{+}^{\vee}$ from the first inclusion and $w^{-1}\left(-\beta^{\vee}\right) \in \Delta_{+}^{\vee}$ from the second one, which leads to the contradiction. Therefore $\lambda \in H_{\alpha^{\vee}}$ for $\alpha \in \Delta^{\prime \prime \vee} \backslash \Delta_{I}^{\vee}$.

Next we give some definitions and facts about convex polytopes (see $[5,37])$ and their triangulation. For a subset $X \subset \mathbb{R}^{N}$, we denote by $\operatorname{Conv}(X)$ the convex hull of $X$. A subset $\mathcal{P} \subset \mathbb{R}^{N}$ is called a convex polytope if $\mathcal{P}=\operatorname{Conv}(X)$ for some finite subset $X \subset \mathbb{R}^{N}$. Let $\mathcal{P}$ be a $d$-dimensional polytope. Let $\mathcal{H}$ be a hyperplane in $\mathbb{R}^{N}$. Then $\mathcal{H}$ divides $\mathbb{R}^{N}$ into two half-spaces. If $\mathcal{P}$ is entirely contained in one of the two closed half-spaces and $\mathcal{P} \cap \mathcal{H} \neq \emptyset$, then $\mathcal{H}$ is called a supporting hyperplane. For a supporting hyperplane $\mathcal{H}$, a subset $\mathcal{F}=\mathcal{P} \cap \mathcal{H} \neq \emptyset$ is called a face of the polytope $\mathcal{P}$. If the dimension of a face $\mathcal{F}$ is $j$, then we call it a $j$-face $\mathcal{F}$. A 0 -face is called a vertex and a $(d-1)$-face a facet. For convenience, we regard $\mathcal{P}$ itself as its unique $d$-face. Let $\operatorname{Vert}(\mathcal{P})$ be the set of the vertices of $\mathcal{P}$. Then

$$
\mathcal{F}=\operatorname{Conv}(\operatorname{Vert}(\mathcal{P}) \cap \mathcal{F}),
$$

for a face $\mathcal{F}$.

It is known that triangulation of a convex polytope can be executed without adding any vertices. Here we give an explicit procedure of a triangulation of $\mathcal{P}$. Number all the vertices of $\mathcal{P}$ as $\mathbf{p}_{1}, \ldots, \mathbf{p}_{k}$. For a face $\mathcal{F}$, by $\mathcal{N}(\mathcal{F})$ we mean the vertex $\mathbf{p}_{j}$ whose index $j$ is the smallest in the vertices belonging to $\mathcal{F}$. A full flag $\Phi$ is defined by the sequence

$$
\Phi: \mathcal{F}_{0} \subset \mathcal{F}_{1} \subset \cdots \subset \mathcal{F}_{d-1} \subset \mathcal{F}_{d}=\mathcal{P},
$$


with $j$-faces $\mathcal{F}_{j}$ such that $\mathcal{N}\left(\mathcal{F}_{j}\right) \notin \mathcal{F}_{j-1}$.

Theorem 2.4 ( [27]). All the collection of the simplexes with vertices $\mathcal{N}\left(\mathcal{F}_{0}\right), \ldots, \mathcal{N}\left(\mathcal{F}_{d-1}\right)$, $\mathcal{N}\left(\mathcal{F}_{d}\right)$ associated with full flags gives a triangulation.

Remark 2.5. This procedure only depends on its face poset structure (see $[5, \S 5]$ ).

For $\mathbf{a}={ }^{t}\left(a_{1}, \ldots, a_{N}\right), \mathbf{b}={ }^{t}\left(b_{1}, \ldots, b_{N}\right) \in \mathbb{C}^{N}$ we define $\mathbf{a} \cdot \mathbf{b}=a_{1} b_{1}+\cdots+a_{N} b_{N}$. The definition of polytopes above is that of "V-polytopes". We mainly deal with another representation of polytopes, "H-polytopes", instead. Namely, consider a bounded subset of the form

$$
\mathcal{P}=\bigcap_{i \in I} \mathcal{H}_{i}^{+} \subset \mathbb{R}^{N}
$$

where $|I|<\infty$ and $\mathcal{H}_{i}^{+}=\left\{\mathbf{x} \in \mathbb{R}^{N} \mid \mathbf{a}_{i} \cdot \mathbf{x} \geq h_{i}\right\}$ with $\mathbf{a}_{i} \in \mathbb{R}^{N}$ and $h_{i} \in \mathbb{R}$. The following theorem is intuitively clear but nontrivial (see, for example, [37, Theorem 1.1]).

Theorem 2.6 (Weyl-Minkowski). H-polytopes are $V$-polytopes and vice versa.

We have a representation of $k$-faces in terms of hyperplanes $\mathcal{H}_{i}=\left\{\mathbf{x} \in \mathbb{R}^{N} \mid \mathbf{a}_{i} \cdot \mathbf{x}=h_{i}\right\}$.

Proposition 2.7. Let $J \subset I$. Assume that $\mathcal{F}=\mathcal{P} \cap \bigcap_{j \in J} \mathcal{H}_{j} \neq \emptyset$. Then $\mathcal{F}$ is a face.

Proof. Let $\mathbf{x} \in \mathcal{P}$. Then $\mathbf{x} \in \bigcap_{j \in J} \mathcal{H}_{j}^{+}$and hence $\mathbf{a}_{j} \cdot \mathbf{x} \geq h_{j}$ for all $j \in J$. Set $\mathbf{a}=\sum_{j \in J} \mathbf{a}_{j}$ and $h=\sum_{j \in J} h_{j}$. Let $\mathcal{H}$ be a hyperplane defined by $\left\{\mathbf{x} \in \mathbb{R}^{N} \mid \mathbf{a} \cdot \mathbf{x}=h\right\}$. Then $\mathbf{a} \cdot \mathbf{x} \geq h$ for $\mathbf{x} \in \mathcal{P}$ and $\mathcal{P} \subset \mathcal{H}^{+}=\left\{\mathbf{x} \in \mathbb{R}^{N} \mid \mathbf{a} \cdot \mathbf{x} \geq h\right\}$.

Let $\mathbf{x} \in \mathcal{P} \cap \mathcal{H}$. Then $\mathbf{a} \cdot \mathbf{x}=h$. Since $\mathbf{a}_{j} \cdot \mathbf{x}=h_{j}+c_{j} \geq h_{j}$ with some $c_{j} \geq 0$ for all $j \in J$, we have $c_{j}=0$ and $\mathbf{x} \in \mathcal{H}_{j}$ for all $j \in J$. Thus $\mathbf{x} \in \mathcal{F}$. It is easily seen that $\mathbf{x} \in \mathcal{F}$ satisfies $\mathbf{x} \in \mathcal{P} \cap \mathcal{H}$. Therefore $\mathcal{F}=\mathcal{P} \cap \mathcal{H}$ and $\mathcal{H}$ is a supporting hyperplane.

Proposition 2.8. Let $\mathcal{H}$ be a supporting hyperplane and $\mathcal{F}=\mathcal{P} \cap \mathcal{H}$ a $k$-face. Then there exists a set of indices $J \subset I$ such that $|J|=(\operatorname{dim} \mathcal{P})-k$ and $\mathcal{F}=\mathcal{P} \cap \bigcap_{j \in J} \mathcal{H}_{j}$.

Proof. Assume $d=N$ without loss of generality. Let $\mathbf{x} \in \mathcal{F}$. Then $\mathbf{x} \in \mathcal{H}$ and hence $\mathbf{x} \in \partial \mathcal{P}$ since $\mathcal{P} \subset \mathcal{H}^{+}$. If $\mathbf{x} \in \mathcal{H}_{i}^{+} \backslash \mathcal{H}_{i}$ for all $i \in I$, then $\mathbf{x}$ is in the interior of $\mathcal{P}$, which contradicts to the above. Thus $\mathbf{x} \in \mathcal{H}_{j}$ for some $j \in I$.

First we assume that $\mathcal{F}=\mathcal{P} \cap \mathcal{H}$ is a facet. Then there exist a subset $\left\{\mathbf{x}_{1}, \ldots, \mathbf{x}_{N}\right\} \subset \mathcal{F}$ such that $\mathbf{x}_{2}-\mathbf{x}_{1}, \ldots, \mathbf{x}_{N}-\mathbf{x}_{1}$ are linearly independent. Let $\mathcal{C}$ be the convex hull of $\left\{\mathbf{x}_{1}, \ldots, \mathbf{x}_{N}\right\}$. We consider that $\mathcal{C} \subset \mathcal{F}$ is equipped with the relative topology. Note that for $\mathbf{x} \in \mathcal{C}$, we have $\mathbf{x} \in \mathcal{H}_{i(\mathbf{x})}$, where $i(\mathbf{x}) \in I$. We show that there exist an open subset $\mathcal{U} \subset \mathcal{C}$ and $i \in I$ such that $\mathcal{H}_{i} \cap \mathcal{U}$ is dense in $\mathcal{U}$. Fix an order $\left\{i_{1}, i_{2}, \ldots\right\}=I$. If $\mathcal{H}_{i_{1}} \cap \mathcal{C}$ is dense in $\mathcal{C}$, then we have done. Hence we assume that it is false. Then there exists an open subset $\mathcal{U}_{1} \subset \mathcal{C}$ such that $\mathcal{H}_{i_{1}} \cap \mathcal{U}_{1}=\emptyset$. Similarly we see that $\mathcal{H}_{i_{2}} \cap \mathcal{U}_{1}$ is dense in $\mathcal{U}_{1}$ unless there exists an open subset $\mathcal{U}_{2} \subset \mathcal{U}_{1}$ such that $\mathcal{H}_{i_{2}} \cap \mathcal{U}_{2}=\emptyset$. Since $|I|<\infty$, repeated application of this argument yields the assertion. Thus there exists a subset $\left\{\mathbf{x}_{1}^{\prime}, \ldots, \mathbf{x}_{N}^{\prime}\right\} \subset \mathcal{H}_{i} \cap \mathcal{U}$ such that $\mathbf{x}_{2}^{\prime}-\mathbf{x}_{1}^{\prime}, \ldots, \mathbf{x}_{N}^{\prime}-\mathbf{x}_{1}^{\prime}$ are linearly independent. Hence we have $\mathcal{F} \subset \mathcal{H}_{i}$ and $\mathcal{H}=\mathcal{H}_{i}$.

For any $k$-face $\mathcal{F}$, there exists a sequence of faces such that

$$
\mathcal{F}=\mathcal{F}_{k} \subset \mathcal{F}_{k+1} \subset \cdots \subset \mathcal{F}_{N-1} \subset \mathcal{P},
$$

where $\mathcal{F}_{j}(k \leq j \leq N-1)$ is a $j$-face. Since $\mathcal{F}_{j}$ is a facet of $\mathcal{F}_{j+1}$, by the induction on dimensions, we have $\mathcal{F}=\mathcal{P} \cap \bigcap_{j \in J} \mathcal{H}_{j}$ for some $J \subset I$ with $|J|=N-k$. 
Lemma 2.9. Let $\mathbf{a}={ }^{t}\left(a_{1}, \ldots, a_{N}\right) \in \mathbb{C}^{N}$ and $\sigma$ be a simplex with vertices $\mathbf{p}_{0}, \ldots, \mathbf{p}_{N} \in$ $\mathbb{R}^{N}$ in general position. Then

$$
\begin{aligned}
\int_{\sigma} e^{\mathbf{a} \cdot \mathbf{x}} d \mathbf{x} & =N ! \operatorname{Vol}(\sigma) \sum_{m=0}^{N} \frac{e^{\mathbf{a} \cdot \mathbf{p}_{m}}}{\prod_{j \neq m} \mathbf{a} \cdot\left(\mathbf{p}_{m}-\mathbf{p}_{j}\right)} \\
& =N ! \operatorname{Vol}(\sigma) T\left(\mathbf{a} \cdot \mathbf{p}_{0}, \ldots, \mathbf{a} \cdot \mathbf{p}_{N}\right),
\end{aligned}
$$

where

$$
\begin{gathered}
T\left(x_{0}, \ldots, x_{N}\right)=\operatorname{det}\left(\begin{array}{ccc}
1 & \cdots & 1 \\
x_{0} & \cdots & x_{N} \\
\vdots & \ddots & \vdots \\
x_{0}^{N-1} & \cdots & x_{N}^{N-1} \\
e^{x_{0}} & \cdots & e^{x_{N}}
\end{array}\right) / \operatorname{det}\left(\begin{array}{ccc}
1 & \cdots & 1 \\
x_{0} & \cdots & x_{N} \\
\vdots & \ddots & \vdots \\
x_{0}^{N-1} & \cdots & x_{N_{N}^{N-1}}^{N} \\
x_{0}^{N} & \cdots & x_{N}^{N}
\end{array}\right), \\
\operatorname{Vol}(\sigma)=\int_{\sigma} 1 d \mathbf{x}=\frac{1}{N !}|\operatorname{det} \tilde{P}|,
\end{gathered}
$$

and

$$
\tilde{P}=\left(\begin{array}{ccc}
1 & \cdots & 1 \\
\mathbf{p}_{0} & \cdots & \mathbf{p}_{N}
\end{array}\right),
$$

with $\mathbf{p}_{j}$ regarded as column vectors.

Proof. Let $\tau$ be the simplex whose vertices consist of the origin and ${ }^{t}\left(0, \ldots,{ }_{1}^{j}, \ldots, 0\right)$, $1 \leq j \leq N$. Then by changing variables from $\mathbf{x}$ to $\mathbf{y}$ as $\mathbf{x}=\mathbf{p}_{0}+P \mathbf{y}$ with the $N \times N$ matrix $P=\left(\mathbf{p}_{1}-\mathbf{p}_{0}, \ldots, \mathbf{p}_{N}-\mathbf{p}_{0}\right)$, we have

$$
\int_{\sigma} e^{\mathbf{a} \cdot \mathbf{x}} d \mathbf{x}=e^{\mathbf{a} \cdot \mathbf{p}_{0}}|\operatorname{det} P| \int_{\tau} e^{\mathbf{b} \cdot \mathbf{y}} d \mathbf{y}
$$

where ${ }^{t} \mathbf{b}=\left(b_{1}, \ldots, b_{N}\right)={ }^{t} \mathbf{a} P$. Since

$$
\begin{aligned}
& \int_{0}^{1-y_{1}-\cdots-y_{m-1}} e^{c+\left(b_{1}-c\right) y_{1}+\cdots+\left(b_{m}-c\right) y_{m}} d y_{m}= \\
& \frac{1}{b_{m}-c}\left(e^{b_{m}+\left(b_{1}-b_{m}\right) y_{1}+\cdots+\left(b_{m-1}-b_{m}\right) y_{m-1}}-e^{c+\left(b_{1}-c\right) y_{1}+\cdots+\left(b_{m-1}-c\right) y_{m-1}}\right),
\end{aligned}
$$

we see that

$$
\int_{\tau} e^{\mathbf{b} \cdot \mathbf{y}} d \mathbf{y}=q_{0}(\mathbf{b})+\sum_{m=1}^{N} q_{m}(\mathbf{b}) e^{b_{m}}
$$

where $q_{j}(\mathbf{b})$ for $0 \leq j \leq N$ are rational functions in $b_{1}, \ldots, b_{N}$. In particular, we have

$$
q_{0}(\mathbf{b})=\frac{(-1)^{N}}{b_{1} \cdots b_{N}}
$$

and hence

$$
\int_{\sigma} e^{\mathbf{a} \cdot \mathbf{x}} d \mathbf{x}=\frac{\left|\operatorname{det}\left(\mathbf{p}_{0}-\mathbf{p}_{1}, \ldots, \mathbf{p}_{0}-\mathbf{p}_{N}\right)\right|}{\left(\mathbf{a} \cdot\left(\mathbf{p}_{0}-\mathbf{p}_{1}\right)\right) \cdots\left(\mathbf{a} \cdot\left(\mathbf{p}_{0}-\mathbf{p}_{N}\right)\right)} e^{\mathbf{a} \cdot \mathbf{p}_{0}}+\sum_{m=1}^{N} \tilde{q}_{m}(\mathbf{a}) e^{\mathbf{a} \cdot \mathbf{p}_{m}}
$$


where $\tilde{q}_{m}(\mathbf{a})$ are certain rational functions in $a_{1}, \ldots, a_{N}$. Since $e^{\mathbf{a} \cdot \mathbf{p}_{j}}$ for $0 \leq j \leq N$ are linearly independent over the field of rational functions in $a_{1}, \ldots, a_{N}$, exchanging the roles of the indices 0 and $j$ in the change of variables in (2.27) yields

$$
\begin{aligned}
\int_{\sigma} e^{\mathbf{a} \cdot \mathbf{x}} d \mathbf{x} & =\sum_{m=0}^{N} \frac{\left|\operatorname{det}\left(\mathbf{p}_{m}-\mathbf{p}_{0}, \ldots, \mathbf{p}_{m}-\mathbf{p}_{m-1}, \mathbf{p}_{m}-\mathbf{p}_{m+1}, \ldots, \mathbf{p}_{m}-\mathbf{p}_{N}\right)\right|}{\prod_{j \neq m} \mathbf{a} \cdot\left(\mathbf{p}_{m}-\mathbf{p}_{j}\right)} e^{\mathbf{a} \cdot \mathbf{p}_{m}} \\
& =N ! \operatorname{Vol}(\sigma) \sum_{m=0}^{N} \frac{e^{\mathbf{a} \cdot \mathbf{p}_{m}}}{\prod_{j \neq m} \mathbf{a} \cdot\left(\mathbf{p}_{m}-\mathbf{p}_{j}\right)}
\end{aligned}
$$

Generally we have

$$
\sum_{m=0}^{N} \frac{e^{x_{m}}}{\prod_{j \neq m}\left(x_{m}-x_{j}\right)}=T\left(x_{0}, \ldots, x_{N}\right)
$$

by the Laplace expansion of the numerator of the right-hand side of (2.24) with respect to the last row and hence the result (2.23).

Although the following lemma is a direct consequence of the second expression of (2.23) with the definition of Schur polynomials and the Jacobi-Trudi formula (see [16]), we give a direct proof for convenience.

Lemma 2.10. Let $\mathbf{a} \in \mathbb{C}^{N}, \sigma$ and $\mathbf{p}_{0}, \ldots, \mathbf{p}_{N} \in \mathbb{R}^{N}$ are the same as in Lemma 2.9. Then the Taylor expansion with respect to $\mathbf{a}$ is given by

$$
\begin{aligned}
\int_{\sigma} e^{\mathbf{a} \cdot \mathbf{x}} d \mathbf{x}=\operatorname{Vol}(\sigma)(1+ & \frac{1}{N+1} \sum_{0 \leq i \leq N} \mathbf{a} \cdot \mathbf{p}_{i}+\cdots \\
& \left.+\frac{N !}{(N+k) !} \sum_{\substack{k_{0}, \ldots, k_{N} \geq 0 \\
k_{0}+\cdots+k_{N}=k}}\left(\mathbf{a} \cdot \mathbf{p}_{0}\right)^{k_{0}} \cdots\left(\mathbf{a} \cdot \mathbf{p}_{N}\right)^{k_{N}}+\cdots\right) .
\end{aligned}
$$

Proof. We recall Dirichlet's integral (see [34, Chap. XII 12.5]) for nonnegative integers $k_{j}$ and a continuous function $g$, that is,

$$
\int_{\tilde{\tau}} y_{0}^{k_{0}} \cdots y_{N}^{k_{N}} g\left(y_{0}+\cdots+y_{N}\right) d y_{0} \cdots d y_{N}=\frac{k_{0} ! \cdots k_{N} !}{\left(N+k_{0}+\cdots+k_{N}\right) !} \int_{0}^{1} g(t) t^{k_{0}+\cdots+k_{N}+N} d t,
$$

where $\tilde{\tau}$ is the $(N+1)$-dimensional simplex with their vertices ${ }^{t}\left(0, \ldots,{ }_{1}^{j}, \ldots, 0\right), 0 \leq j \leq N$ and the origin. This formula is easily obtained by repeated application of the beta integral.

We calculate

$$
f(\mathbf{a})=\frac{1}{k !} \int_{\sigma}(\mathbf{a} \cdot \mathbf{x})^{k} d \mathbf{x}
$$

By multiplying

$$
1=(k+1) \int_{0}^{1} s^{k} d s
$$

and changing variables as $\mathbf{x}^{\prime}=s \mathbf{x}$, we obtain

$$
f(\mathbf{a})=\frac{k+1}{k !} \int_{\tilde{\sigma}}\left(\mathbf{a} \cdot \mathbf{x}^{\prime}\right)^{k} s^{-N} d s d \mathbf{x}^{\prime}
$$


where $\tilde{\sigma}=\bigcup_{0 \leq s \leq 1}\left(\begin{array}{c}s \\ s \sigma\end{array}\right)$ is an $(N+1)$-dimensional simplex. Again we change variables as $\tilde{P} \tilde{\mathbf{y}}=\tilde{\mathbf{x}}=\left(\begin{array}{c}s \\ \mathbf{x}^{\prime}\end{array}\right)$. Then

$$
\begin{aligned}
f(\mathbf{a}) & =\frac{k+1}{k !}|\operatorname{det} \tilde{P}| \int_{\tilde{\tau}}\left(\sum_{j=0}^{N}\left(\mathbf{a} \cdot \mathbf{p}_{j}\right) y_{j}\right)^{k}\left(\sum_{j=0}^{N} y_{j}\right)^{-N} d \tilde{\mathbf{y}} \\
& =\frac{k+1}{k !}|\operatorname{det} \tilde{P}| \sum_{\substack{k_{0}, \ldots, k_{N} \geq 0 \\
k_{0}+\cdots+k_{N}=k}}\left(\mathbf{a} \cdot \mathbf{p}_{0}\right)^{k_{0}} \cdots\left(\mathbf{a} \cdot \mathbf{p}_{N}\right)^{k_{N}} \frac{k !}{k_{0} ! \cdots k_{N} !} \int_{\tilde{\tau}} y_{0}^{k_{0}} \cdots y_{N}^{k_{N}}\left(\sum_{j=0}^{N} y_{j}\right)^{-N} d \tilde{\mathbf{y}},
\end{aligned}
$$

where $\tilde{\mathbf{y}}={ }^{t}\left(y_{0}, \ldots, y_{N}\right)$. Hence applying (2.35) , we obtain

$$
\begin{aligned}
f(\mathbf{a}) & =\frac{k+1}{k !}|\operatorname{det} \tilde{P}| \sum_{\substack{k_{0}, \ldots, k_{N} \geq 0 \\
k_{0}+\cdots+k_{N}=k}}\left(\mathbf{a} \cdot \mathbf{p}_{0}\right)^{k_{0}} \cdots\left(\mathbf{a} \cdot \mathbf{p}_{N}\right)^{k_{N}} \frac{k !}{(N+k) !} \frac{1}{k+1} \\
& =\operatorname{Vol}(\sigma) \frac{N !}{(N+k) !} \sum_{\substack{k_{0}, \ldots, k_{N} \geq 0 \\
k_{0}+\cdots+k_{N}=k}}\left(\mathbf{a} \cdot \mathbf{p}_{0}\right)^{k_{0}} \cdots\left(\mathbf{a} \cdot \mathbf{p}_{N}\right)^{k_{N}} .
\end{aligned}
$$

It should be remarked that $\mathbf{a} \cdot \mathbf{p}_{j}$ for $0 \leq j \leq N$ are not linearly independent. Thus the coefficients with respect to them are not unique. Lemma 2.10 is a special and exact case of the following lemma.

Lemma 2.11. Let $\mathbf{a} \in \mathbb{C}^{N}, \sigma$ and $\mathbf{p}_{0}, \ldots, \mathbf{p}_{N} \in \mathbb{R}^{N}$ are the same as in Lemma 2.9. Then for $\mathbf{b} \in \mathbb{C}^{N}$, the coefficients of total degree $k$ with respect to $\mathbf{a}$ of the Taylor expansion of

$$
\int_{\sigma} e^{(\mathbf{a}+\mathbf{b}) \cdot \mathbf{x}} d \mathbf{x}
$$

are holomorphic functions in $\mathbf{b}$ of the form

$$
\operatorname{Vol}(\sigma) \sum_{m=0}^{N} \sum_{\substack{k_{0}, \ldots, k_{N} \geq 0 \\ k_{0}+\cdots+k_{N}=k}} c_{m, k_{0}, \ldots, k_{N}} \frac{e^{\mathbf{b} \cdot \mathbf{p}_{m}}}{\prod_{j \neq m}\left(\mathbf{b} \cdot\left(\mathbf{p}_{m}-\mathbf{p}_{j}\right)\right)^{k_{j}+1}}
$$

where $c_{m, k_{0}, \ldots, k_{N}} \in \mathbb{Q}$. 
Proof. We assume $\left|\mathbf{a} \cdot\left(\mathbf{p}_{m}-\mathbf{p}_{j}\right)\right|<\left|\mathbf{b} \cdot\left(\mathbf{p}_{m}-\mathbf{p}_{j}\right)\right|$ for all $j \neq m$. Then we have

$$
\begin{aligned}
& \frac{e^{(\mathbf{a}+\mathbf{b}) \cdot \mathbf{p}_{m}}}{\prod_{j \neq m}(\mathbf{a}+\mathbf{b}) \cdot\left(\mathbf{p}_{m}-\mathbf{p}_{j}\right)} \\
& =\frac{e^{\mathbf{b} \cdot \mathbf{p}_{m}}}{\prod_{j \neq m} \mathbf{b} \cdot\left(\mathbf{p}_{m}-\mathbf{p}_{j}\right)} \frac{e^{\mathbf{a} \cdot \mathbf{p}_{m}}}{\prod_{j \neq m}\left(1+\frac{\mathbf{a} \cdot\left(\mathbf{p}_{m}-\mathbf{p}_{j}\right)}{\mathbf{b} \cdot\left(\mathbf{p}_{m}-\mathbf{p}_{j}\right)}\right)} \\
& =\frac{e^{\mathbf{b} \cdot \mathbf{p}_{m}}}{\prod_{j \neq m} \mathbf{b} \cdot\left(\mathbf{p}_{m}-\mathbf{p}_{j}\right)} e^{\mathbf{a} \cdot \mathbf{p}_{m}} \prod_{j \neq m} \sum_{k_{j}=0}^{\infty}\left(-\frac{\mathbf{a} \cdot\left(\mathbf{p}_{m}-\mathbf{p}_{j}\right)}{\mathbf{b} \cdot\left(\mathbf{p}_{m}-\mathbf{p}_{j}\right)}\right)^{k_{j}} \\
& =\sum_{k=0}^{\infty} \sum_{\substack{k_{0}, \ldots, k_{N} \geq 0 \\
k_{0}+\cdots+k_{N}=k}} \frac{e^{\mathbf{b} \cdot \mathbf{p}_{m}}}{\prod_{j \neq m}\left(\mathbf{b} \cdot\left(\mathbf{p}_{m}-\mathbf{p}_{j}\right)\right)^{k_{j}+1}} \frac{\left(\mathbf{a} \cdot \mathbf{p}_{m}\right)^{k_{m}}}{k_{m} !} \prod_{j \neq m}\left(\mathbf{a} \cdot\left(\mathbf{p}_{j}-\mathbf{p}_{m}\right)\right)^{k_{j}} .
\end{aligned}
$$

Applying this result to the second member of (2.23) with $\mathbf{a}$ replaced by $\mathbf{a}+\mathbf{b}$, we see that the coefficients of total degree $k$ are of the form (2.42). The holomorphy with respect to $\mathbf{a}$ and $\mathbf{b}$ follows from the original integral form (2.41). Therefore (2.42) is valid for the whole space with removable singularities.

\section{General functional Relations}

The purpose of this section is to give a very general formulation of functional relations. For $f, g \in \mathfrak{F}(P)$ and $I, J \subset\{1, \ldots, r\}$, we define

$$
\zeta(f, g ; J ; \Delta)=\sum_{\lambda \in P_{J++} \backslash H_{g}} \frac{f(\lambda)}{g(\lambda)},
$$

and

$$
S(f, g ; I ; \Delta)=\sum_{\lambda \in \iota^{*-1}\left(P_{I+}\right) \backslash H_{g}} \frac{f(\lambda)}{g(\lambda)} .
$$

We assume that (3.2) is absolutely convergent for a fixed $I$. By (2.15) and Lemma 2.2. we have id $\in W^{I}$ and $P_{+} \subset \iota^{*-1}\left(P_{I+}\right)$. Hence (3.1) is also absolutely convergent for all $J$ since $P_{J++} \subset P_{+}$by (2.13).

For $s \in \mathbb{C}, \Re s>1$ and $x, c \in \mathbb{R}$ let

$$
L_{s}(x, c)=-\frac{\Gamma(s+1)}{(2 \pi \sqrt{-1})^{s}} \sum_{\substack{n \in \mathbb{Z} \\ n+c \neq 0}} \frac{e^{2 \pi \sqrt{-1}(n+c) x}}{(n+c)^{s}} .
$$

Let $D_{I^{c}}$ be a finite subset of $\left(Q^{\vee} \backslash\{0\}\right) \oplus \mathbb{R} \delta \subset \widehat{V}$. Then any element of $\gamma \in D_{I^{c}}$ can be written as $\gamma=\eta_{\gamma}+c_{\gamma} \delta\left(\eta_{\gamma} \in Q^{\vee} \backslash\{0\}, c_{\gamma} \in \mathbb{R}\right)$. We assume that $D_{I^{c}}$ contains $B_{I^{c}}=\left\{\gamma_{i}\right\}_{i \in I^{c}}$ where $\gamma_{i}=\eta_{i}+c_{i} \delta$ for $i \in I^{c}$ such that $\left\{\eta_{i}\right\}_{i \in I^{c}}$ forms a basis of $Q_{I^{c}}^{\vee}$ and $c_{i} \in \mathbb{R}$. Let $\left\{\mu_{i}\right\}_{i \in I^{c}} \subset P_{I^{c}}$ be a basis dual to $\left\{\eta_{i}\right\}_{i \in I^{c}}$.

Theorem 3.1. Let $s_{\gamma} \in \mathbb{C}$ with $\Re s_{\gamma}>1$ for $\gamma \in D_{I^{c}}$ and let $\mathbf{y} \in V_{I^{c}}$. We assume that

$$
\begin{aligned}
& f(\lambda+\mu)=f(\lambda) e^{2 \pi \sqrt{-1}\langle\mathbf{y}, \mu\rangle}, \\
& g(\lambda+\mu)=g^{\sharp}(\lambda) \prod_{\gamma \in D_{I^{c}}} \gamma(\lambda+\mu)^{s_{\gamma}},
\end{aligned}
$$


for any $\lambda \in P_{I+}$ and any $\mu \in P_{I^{c}}$, where $g^{\sharp} \in \mathfrak{F}\left(P_{I}\right)$. (Hence $f$ depends on $\mathbf{y}$, and $g$ depends on $s_{\gamma}$ 's.) Then

(i) We have

$$
\begin{aligned}
S(f, g ; I ; \Delta) & =\sum_{w \in W^{I}} \sum_{J \subset\{1, \ldots, r\}} \frac{1}{N_{w, J}} \zeta\left(w^{-1} f, w^{-1} g ; J ; \Delta\right) \\
& =\sum_{J \subset I} \zeta\left(f \cdot f^{\sharp}, g^{\sharp} ; J ; \Delta\right),
\end{aligned}
$$

where

$$
N_{w, J}=\left|w W\left(\Delta_{J^{c}}\right) \cap W^{I}\right|
$$

and $f^{\sharp} \in \mathfrak{F}\left(P_{I}\right)$ is defined by

$$
\begin{aligned}
f^{\sharp}(\lambda)=( & -1)^{\left|D_{I^{c}}\right|} e^{-2 \pi \sqrt{-1}\langle\mathbf{y}, \nu\rangle}\left(\prod_{\gamma \in D_{I^{c}}} \frac{(2 \pi \sqrt{-1})^{s_{\gamma}}}{\Gamma\left(s_{\gamma}+1\right)}\right) \\
& \times \int_{0}^{1} \ldots \int_{0}^{1} \exp \left(-2 \pi \sqrt{-1} \sum_{\gamma \in D_{I^{c} \backslash B_{I^{c}}}} \gamma(\lambda-\nu) x_{\gamma}\right)\left(\prod_{\gamma \in D_{I^{c} \backslash B_{I^{c}}}} L_{s_{\gamma}}\left(x_{\gamma}, c_{\gamma}\right)\right) \\
& \times\left(\prod_{i \in I^{c}} L_{s_{\gamma_{i}}}\left(\left\langle\mathbf{y}, \mu_{i}\right\rangle-\sum_{\gamma \in D_{I^{c}} \backslash B_{I^{c}}} x_{\gamma}\left\langle\eta_{\gamma}, \mu_{i}\right\rangle, c_{i}\right)\right) \prod_{\gamma \in D_{I^{c}} \backslash B_{I^{c}}} d x_{\gamma},
\end{aligned}
$$

and

$$
\nu=\sum_{i \in I^{c}} c_{i} \mu_{i} \in \mathbb{R} \otimes P_{I^{c}}
$$

(ii) The second member of (3.6) consists of $2^{r}\left(W(\Delta): W\left(\Delta_{I}\right)\right)$ terms.

(iii) If $H_{\Delta \vee \backslash \Delta_{I}^{\vee}} \subset H_{g}$, then $\zeta\left(w^{-1} f, w^{-1} g ; J ; \Delta\right)=0$ unless $N_{w, J}=1$.

Proof. First we claim that for $w, w^{\prime} \in W^{I}$ and $\lambda \in w P_{J++}$, we have $\lambda \in w^{\prime} P_{J++}$ if and only if $w^{\prime} \in w W\left(\Delta_{J^{c}}\right)$. In fact, $\lambda \in w P_{J++} \cap w^{\prime} P_{J++}$ implies $w^{\prime-1} \lambda \in P_{J++}$ and $\left(w^{-1} w^{\prime}\right) w^{-1} \lambda \in P_{J++}$, and hence $w^{-1} w^{\prime}$ stabilizes $P_{J++}=P_{+} \cap C_{J}$. Therefore $w^{-1} w^{\prime} \in$ $W\left(\Delta_{J^{c}}\right)$. The converse statement is shown by reversing the arguments and we have the claim. By using this claim, Lemma 2.2 and the decomposition (2.13), we have

$$
\begin{aligned}
S(f, g ; I ; \Delta) & =\sum_{\lambda \in \iota^{*-1}\left(P_{I+}\right) \backslash H_{g}} \frac{f(\lambda)}{g(\lambda)} \\
& =\sum_{w \in W^{I}} \sum_{J \subset\{1, \ldots, r\}} \frac{1}{N_{w, J}} \sum_{\lambda \in w P_{J++} \backslash H_{g}} \frac{f(\lambda)}{g(\lambda)} .
\end{aligned}
$$


Therefore

$$
\begin{aligned}
S(f, g ; I ; \Delta) & =\sum_{w \in W^{I}} \sum_{J \subset\{1, \ldots, r\}} \frac{1}{N_{w, J}} \sum_{\lambda \in P_{J++} \backslash w^{-1} H_{g}} \frac{f(w \lambda)}{g(w \lambda)} \\
& =\sum_{w \in W^{I}} \sum_{J \subset\{1, \ldots, r\}} \frac{1}{N_{w, J}} \sum_{\lambda \in P_{J++} \backslash H_{w^{-1} g}} \frac{\left(w^{-1} f\right)(\lambda)}{\left(w^{-1} g\right)(\lambda)} \\
& =\sum_{w \in W^{I}} \sum_{J \subset\{1, \ldots, r\}} \frac{1}{N_{w, J}} \zeta\left(w^{-1} f, w^{-1} g ; J ; \Delta\right),
\end{aligned}
$$

where the last member consists of $2^{r}\left(W(\Delta): W\left(\Delta_{I}\right)\right)$ terms since the cardinality of the power set of $\{1, \ldots, r\}$ is $2^{r}$ and $\left|W^{I}\right|=\left(W(\Delta): W\left(\Delta_{I}\right)\right)$ by Lemma 2.1, which implies the statement (ii).

Assume that $\gamma=\eta_{\gamma}+c_{\gamma} \delta \in\left(Q^{\vee} \backslash\{0\}\right) \oplus \mathbb{R} \delta$ and $\lambda \in P$. Then $\gamma(\lambda)=\left\langle\eta_{\gamma}, \lambda\right\rangle+c_{\gamma} \in \mathbb{Z}+c_{\gamma}$ and for $\gamma(\lambda) \neq 0$, we have

$$
\begin{aligned}
\frac{1}{\gamma(\lambda)^{s_{\gamma}}} & =\sum_{\substack{n_{\gamma} \in \mathbb{Z} \\
n_{\gamma}+c_{\gamma} \neq 0}} \int_{0}^{1} \frac{e^{2 \pi \sqrt{-1}\left(n_{\gamma}+c_{\gamma}-\gamma(\lambda)\right) x_{\gamma}}}{\left(n_{\gamma}+c_{\gamma}\right)^{s_{\gamma}}} d x_{\gamma} \\
& =\int_{0}^{1} \sum_{\substack{n_{\gamma} \in \mathbb{Z} \\
n_{\gamma}+c_{\gamma} \neq 0}} \frac{e^{2 \pi \sqrt{-1}\left(n_{\gamma}+c_{\gamma}-\gamma(\lambda)\right) x_{\gamma}}}{\left(n_{\gamma}+c_{\gamma}\right)^{s_{\gamma}}} d x_{\gamma} \\
& =-\frac{(2 \pi \sqrt{-1})^{s_{\gamma}}}{\Gamma\left(s_{\gamma}+1\right)} \int_{0}^{1} L_{s_{\gamma}}\left(x_{\gamma}, c_{\gamma}\right) e^{-2 \pi \sqrt{-1} \gamma(\lambda) x_{\gamma}} d x_{\gamma}
\end{aligned}
$$

where we have used the absolute convergence because of $\Re s_{\gamma}>1$.

By using (3.5), (3.4) and Lemma 2.2, we have

$$
S(f, g ; I ; \Delta)=\sum_{\lambda \in P_{I+}} \sum_{\substack{\mu \in P_{I^{c}} \\ \lambda+\mu \notin H_{g}}} \frac{f(\lambda)}{g^{\sharp}(\lambda)} e^{2 \pi \sqrt{-1}\langle\mathbf{y}, \mu\rangle}\left(\prod_{\gamma \in D_{I^{c}}} \frac{1}{\gamma(\lambda+\mu)^{s_{\gamma}}}\right) .
$$

Applying (3.12) to the right-hand side of the above, we obtain

$$
\begin{aligned}
S(f, g ; I ; \Delta)= & \sum_{\lambda \in P_{I+}} \sum_{\substack{\mu \in P_{I^{c}} \\
\lambda+\mu \notin H_{g}}} \frac{f(\lambda)}{g^{\sharp}(\lambda)} e^{2 \pi \sqrt{-1}\langle\mathbf{y}, \mu\rangle}\left(\prod_{\gamma \in B_{I^{c}}} \frac{1}{\gamma(\lambda+\mu)^{s_{\gamma}}}\right) \\
& \times\left(\prod_{\substack{\gamma \in D_{I^{c}} \backslash B_{I^{c}}}}-\frac{(2 \pi \sqrt{-1})^{s_{\gamma}}}{\Gamma\left(s_{\gamma}+1\right)} \int_{0}^{1} L_{s_{\gamma}}\left(x_{\gamma}, c_{\gamma}\right) e^{-2 \pi \sqrt{-1} \gamma(\lambda+\mu) x_{\gamma}} d x_{\gamma}\right) .
\end{aligned}
$$

Note that if $\gamma(\lambda)=0$, then $c_{\gamma} \in \mathbb{Z}$ and the last member of (3.12) vanishes. Hence we may add the case $\gamma(\lambda+\mu)=0$ for $\gamma \in D_{I^{c}} \backslash B_{I^{c}}$ in the above. Therefore by using 
$H_{g}=H_{g^{\sharp}} \cup H_{D_{I^{c}} \backslash B_{I^{c}}} \cup H_{B_{I^{c}}}$ and putting $\mu=\sum_{i \in I^{c}} n_{i} \mu_{i}\left(n_{i} \in \mathbb{Z}\right)$, we have

$$
\begin{aligned}
& S(f, g ; I ; \Delta) \\
& =\left(\prod_{\gamma \in D_{I^{c}} \backslash B_{I^{c}}}-\frac{(2 \pi \sqrt{-1})^{s_{\gamma}}}{\Gamma\left(s_{\gamma}+1\right)}\right) \sum_{\lambda \in P_{I+} \backslash H_{g^{\sharp}}} \frac{f(\lambda)}{g^{\sharp}(\lambda)} \\
& \times \sum_{\substack{n_{i} \in \mathbb{Z} \\
n_{i}+c_{i} \neq 0 \\
i \in I^{c}}} \int_{0}^{1} \cdots \int_{0}^{1} \exp \left(-2 \pi \sqrt{-1} \sum_{\gamma \in D_{I^{c}} \backslash B_{I^{c}}} \gamma(\lambda) x_{\gamma}\right)\left(\prod_{\gamma \in D_{I^{c}} \backslash B_{I^{c}}} L_{s_{\gamma}}\left(x_{\gamma}, c_{\gamma}\right)\right) \\
& \times \prod_{i \in I^{c}} \frac{\exp \left(2 \pi \sqrt{-1}\left(\left\langle\mathbf{y}, \mu_{i}\right\rangle-\sum_{\gamma \in D_{I^{c}} \backslash B_{I^{c}}} x_{\gamma}\left\langle\eta_{\gamma}, \mu_{i}\right\rangle\right) n_{i}\right)}{\left(n_{i}+c_{i}\right)^{s \gamma_{i}}} \prod_{\gamma \in D_{I^{c}} \backslash B_{I^{c}}} d x_{\gamma} \\
& =(-1)^{\left|D_{I^{c}}\right|} e^{-2 \pi \sqrt{-1}\langle\mathbf{y}, \nu\rangle}\left(\prod_{\gamma \in D_{I^{c}}} \frac{(2 \pi \sqrt{-1})^{s_{\gamma}}}{\Gamma\left(s_{\gamma}+1\right)}\right) \sum_{\lambda \in P_{I+} \backslash H_{g^{\sharp}}} \frac{f(\lambda)}{g^{\sharp}(\lambda)} \\
& \times \int_{0}^{1} \ldots \int_{0}^{1} \exp \left(-2 \pi \sqrt{-1} \sum_{\gamma \in D_{I^{c}} \backslash B_{I^{c}}} \gamma(\lambda-\nu) x_{\gamma}\right)\left(\prod_{\gamma \in D_{I^{c}} \backslash B_{I^{c}}} L_{s_{\gamma}}\left(x_{\gamma}, c_{\gamma}\right)\right) \\
& \times\left(\prod_{i \in I^{c}} L_{s_{\gamma_{i}}}\left(\left\langle\mathbf{y}, \mu_{i}\right\rangle-\sum_{\gamma \in D_{I^{c}} \backslash B_{I^{c}}} x_{\gamma}\left\langle\eta_{\gamma}, \mu_{i}\right\rangle, c_{i}\right)\right) \prod_{\gamma \in D_{I^{c}} \backslash B_{I^{c}}} d x_{\gamma},
\end{aligned}
$$

which is equal to the third member of (3.6), because $P_{I+}=\bigcup_{J \subset I} P_{J++}$. Hence the statement (i).

As for the last claim (iii) of the theorem, we first note that $N_{w, J}>1$ if and only if $w P_{J++} \subset H_{\Delta^{\vee} \backslash \Delta_{I}^{\vee}}$. This follows from Lemma 2.3 and the definition of $N_{w, J}$, with noting the claim proved on the first line of the present proof. Now assume $H_{\Delta^{\vee} \backslash \Delta_{I}^{\vee}} \subset H_{g}$. Then, if $N_{w, J}>1$, we have $w P_{J++} \subset H_{g}$. This implies $\zeta\left(w^{-1} f, w^{-1} g ; J ; \Delta\right)=0$, because the definition (3.1) is an empty sum in this case.

In the present paper we mainly discuss the case when $D_{I^{c}} \subset Q^{\vee}$. Nevertheless we give the above generalized form of Theorem 3.1, by which we can treat the case of zeta-functions of Hurwitz-type.

For a real number $x$, let $\{x\}$ denote its fractional part $x-[x]$. If $s=k \geq 2$ and $c$ are integers, then it is known (see [1]) that

$$
\begin{aligned}
L_{k}(x, c) & =-\frac{k !}{(2 \pi \sqrt{-1})^{k}} \sum_{n \in \mathbb{Z} \backslash\{0\}} \frac{e^{2 \pi \sqrt{-1} n x}}{n^{k}} \\
& =B_{k}(\{x\}),
\end{aligned}
$$

where $B_{k}(x)$ is the $k$-th Bernoulli polynomial. Thus if all $L_{s}(x, c)$ in the integrand on the right-hand side of (3.8) are written in terms of Bernoulli polynomials, then we have a chance to obtain an explicit form of the right-hand side of (3.8). We calculate the integral in question through a generating function instead of a direct calculation. The result will be stated in Theorem 3.5 below.

Remark 3.2. When $s_{\gamma}=1$, the argument (3.12) is not valid, because the series is conditionally convergent. Hence on the right-hand side of (3.15), $s_{\gamma_{i}}=1$ for $i \in I^{c}$ is not 
allowed. However for $n \in \mathbb{Z} \backslash\{0\}$, we have

$$
\frac{1}{n}=(-2 \pi \sqrt{-1}) \int_{0}^{1} B_{1}(x) e^{-2 \pi \sqrt{-1} n x} d x
$$

where the right-hand side vanishes if $n=0$. Thus the value $s_{\gamma}=1$ is also allowed for $\gamma \in\left(D_{I^{c}} \backslash B_{I^{c}}\right) \cap \widehat{Q}^{\vee}$ on the right-hand side of (3.14) and hence in Theorem 3.1.

Let $M, N \in \mathbb{N}, \mathbf{k}=\left(k_{l}\right)_{1 \leq l \leq M+N} \in \mathbb{N}_{0}^{M+N}, \mathbf{y}=\left(y_{i}\right)_{1 \leq i \leq M} \in \mathbb{R}^{M}$ and $b_{j} \in \mathbb{C}, c_{i j} \in \mathbb{R}$ for $1 \leq i \leq M$ and $1 \leq j \leq N$. Let

$$
P(\mathbf{k}, \mathbf{y})=\int_{0}^{1} \ldots \int_{0}^{1} \exp \left(\sum_{j=1}^{N} b_{j} x_{j}\right)\left(\prod_{j=1}^{N} B_{k_{j}}\left(x_{j}\right)\right)\left(\prod_{i=1}^{M} B_{k_{N+i}}\left(\left\{y_{i}-\sum_{j=1}^{N} c_{i j} x_{j}\right\}\right)\right) \prod_{j=1}^{N} d x_{j} .
$$

For $\mathbf{t}=\left(t_{l}\right)_{1 \leq l \leq M+N} \in \mathbb{C}^{M+N}$, we define a generating function of $P(\mathbf{k}, \mathbf{y})$ by

$$
F(\mathbf{t}, \mathbf{y})=\sum_{\mathbf{k} \in \mathbb{N}_{0}^{M+N}} P(\mathbf{k}, \mathbf{y}) \prod_{j=1}^{M+N} \frac{t_{j}^{k_{j}}}{k_{j} !}
$$

Lemma 3.3. (i) The generating function $F(\mathbf{t}, \mathbf{y})$ is absolutely convergent, uniformly on $\mathcal{D}_{R} \times \mathbb{R}^{M}$ where $\mathcal{D}_{R}=\{t \in \mathbb{C}|| t \mid \leq R\}^{M+N}$ with $0<R<2 \pi$.

(ii) The function $F(\cdot, \mathbf{y})$ is analytically continued to a meromorphic function in $\mathbf{t}$ on the whole space $\mathbb{C}^{M+N}$ and we have

$$
\begin{aligned}
F(\mathbf{t}, \mathbf{y})= & \left(\prod_{j=1}^{M+N} \frac{t_{j}}{e^{t_{j}}-1}\right) \int_{0}^{1} \ldots \int_{0}^{1}\left(\prod_{j=1}^{N} \exp \left(\left(b_{j}+t_{j}\right) x_{j}\right)\right) \\
& \times\left(\prod_{i=1}^{M} \exp \left(t_{N+i}\left\{y_{i}-\sum_{j=1}^{N} c_{i j} x_{j}\right\}\right)\right) \prod_{j=1}^{N} d x_{j} \\
= & \left(\prod_{j=1}^{M+N} \frac{t_{j}}{e^{t_{j}}-1}\right) \sum_{m_{1}=c_{1}^{-}}^{c_{1}^{+}} \cdots \sum_{m_{M}=c_{M}^{-}}^{c_{M}^{+}} \exp \left(\sum_{i=1}^{M} t_{N+i}\left(\left\{y_{i}\right\}+m_{i}\right)\right) \\
& \times \int_{\mathcal{P}_{\mathbf{m}, \mathbf{y}}} \exp \left(\sum_{j=1}^{N}\left(a_{j}+b_{j}\right) x_{j}\right) \prod_{j=1}^{N} d x_{j},
\end{aligned}
$$

where $c_{i}^{+}$is the minimum integer satisfying $c_{i}^{+} \geq \sum_{\substack{1 \leq j \leq N \\ c_{i j}>0}} c_{i j}$ and $c_{i}^{-}$is the maximum integer satisfying $c_{i}^{-} \leq \sum_{\substack{1 \leq j \leq N \\ c_{i j}<0}} c_{i j}$ and

$$
\mathcal{P}_{\mathbf{m}, \mathbf{y}}=\left\{\begin{array}{l|l}
\mathbf{x}=\left(x_{j}\right)_{1 \leq j \leq N} & \begin{array}{l}
0 \leq x_{j} \leq 1, \quad(1 \leq j \leq N) \\
\left\{y_{i}\right\}+m_{i}-1 \leq \sum_{j=1}^{N} c_{i j} x_{j} \leq\left\{y_{i}\right\}+m_{i}, \quad(1 \leq i \leq M)
\end{array}
\end{array}\right\},
$$

which is a convex polytope. 
Proof. (i) Fix $R^{\prime} \in \mathbb{R}$ such that $R<R^{\prime}<2 \pi$. Then by the Cauchy integral formula

$$
\frac{B_{k}(x)}{k !}=\frac{1}{2 \pi \sqrt{-1}} \int_{|z|=R^{\prime}} \frac{z e^{z x}}{e^{z}-1} \frac{d z}{z^{k+1}}
$$

we have for $0 \leq x \leq 1$

$$
\left|\frac{B_{k}(x)}{k !}\right| \leq \frac{1}{2 \pi} \int_{|z|=R^{\prime}}\left|\frac{z e^{z x}}{e^{z}-1}\right| \frac{|d z|}{R^{\prime k+1}} \leq \frac{C_{R^{\prime}}}{R^{\prime k}},
$$

where

$$
C_{R^{\prime}}=\max \left\{\left|\frac{z e^{z x}}{e^{z}-1}\right||| z \mid=R^{\prime}, 0 \leq x \leq 1\right\} .
$$

Therefore

$$
\begin{aligned}
\mid P(\mathbf{k}, \mathbf{y}) \prod_{j=1}^{M+N} \frac{t_{j}^{k_{j}}}{k_{j} !} \leq & \left(\prod_{j=1}^{M+N}\left|t_{j}\right|^{k_{j}}\right) \int_{0}^{1} \ldots \int_{0}^{1} \mid \exp \left(\sum_{j=1}^{N} b_{j} x_{j}\right)\left(\prod_{j=1}^{N} \frac{B_{k_{j}}\left(x_{j}\right)}{k_{j} !}\right) \\
& \times\left(\prod_{i=1}^{M} \frac{B_{k_{N+i}}\left(\left\{y_{i}-\sum_{j=1}^{N} c_{i j} x_{j}\right\}\right)}{k_{N+i} !}\right) \mid \prod_{j=1}^{N} d x_{j} \\
\leq & C\left(\prod_{j=1}^{M+N} R^{k_{j}}\right) \int_{0}^{1} \ldots \int_{0}^{1} C_{R^{\prime}}^{M+N} \prod_{j=1}^{M+N} \frac{1}{R^{\prime k_{j}}} \prod_{j=1}^{N} d x_{j} \\
= & C C_{R^{\prime}}^{M+N} \prod_{j=1}^{M+N}\left(\frac{R}{R^{\prime}}\right)^{k_{j}}
\end{aligned}
$$

where $C=\exp \left(\sum_{j=1}^{N}\left|\Re b_{j}\right|\right)$. Since

$$
\sum_{\mathbf{k} \in \mathbb{N}_{0}^{M+N}} C C_{R^{\prime}}^{M+N} \prod_{j=1}^{M+N}\left(\frac{R}{R^{\prime}}\right)^{k_{j}}=C C_{R^{\prime}}^{M+N} \prod_{j=1}^{M+N} \frac{1}{1-R / R^{\prime}}<\infty
$$

we have the uniform and absolute convergence of $F(\mathbf{t}, \mathbf{y})$. 
(ii) Noting the absolute convergence shown in (i), we obtain

$$
\begin{aligned}
F(\mathbf{t}, \mathbf{y})= & \sum_{\mathbf{k} \in \mathbb{N}_{0}^{M+N}} P(\mathbf{k}, \mathbf{y}) \prod_{j=1}^{M+N} \frac{t_{j}^{k_{j}}}{k_{j} !} \\
= & \sum_{\mathbf{k} \in \mathbb{N}_{0}^{M+N}} \int_{0}^{1} \ldots \int_{0}^{1} \exp \left(\sum_{j=1}^{N} b_{j} x_{j}\right)\left(\prod_{j=1}^{N} B_{k_{j}}\left(x_{j}\right) \frac{t_{j}^{k_{j}}}{k_{j} !}\right) \\
& \times\left(\prod_{i=1}^{M} B_{k_{N+i}}\left(\left\{y_{i}-\sum_{j=1}^{N} c_{i j} x_{j}\right\}\right) \frac{t_{N+i}^{k_{N+i}}}{k_{N+i} !}\right) \prod_{j=1}^{N} d x_{j} \\
= & \left(\prod_{j=1}^{M+N} \frac{t_{j}}{e^{t_{j}}-1}\right) \int_{0}^{1} \ldots \int_{0}^{1} \exp \left(\sum_{j=1}^{N} b_{j} x_{j}\right)\left(\prod_{j=1}^{N} \exp \left(t_{j} x_{j}\right)\right) \\
& \times\left(\prod_{i=1}^{M} \exp \left(t_{N+i}\left\{y_{i}-\sum_{j=1}^{N} c_{i j} x_{j}\right\}\right)\right) \prod_{j=1}^{N} d x_{j} \\
= & \left(\prod_{j=1}^{M+N} \frac{t_{j}}{e^{t_{j}}-1}\right) \int_{0}^{1} \ldots \int_{0}^{1} \exp \left(\sum_{j=1}^{N}\left(b_{j}+t_{j}-\sum_{i=1}^{M} t_{N+i} c_{i j}\right) x_{j}\right) \\
& \times \exp \left(\sum_{i=1}^{M} t_{N+i}\left(y_{i}-\left[y_{i}-\sum_{j=1}^{N} c_{i j} x_{j}\right]\right)\right) \prod_{j=1}^{N} d x_{j} .
\end{aligned}
$$

Here the inequality

$$
y_{i}-c_{i}^{+} \leq y_{i}-\sum_{j=1}^{N} c_{i j} x_{j} \leq y_{i}-c_{i}^{-}
$$

implies

$$
\left\{y_{i}\right\}+c_{i}^{-} \leq y_{i}-\left[y_{i}-\sum_{j=1}^{N} c_{i j} x_{j}\right] \leq\left\{y_{i}\right\}+c_{i}^{+},
$$

and for $m_{i} \in \mathbb{Z}$, the region of $\mathbf{x}$ satisfying

$$
y_{i}-\left[y_{i}-\sum_{j=1}^{N} c_{i j} x_{j}\right]=\left\{y_{i}\right\}+m_{i}
$$

is given by

$$
\left\{y_{i}\right\}+m_{i}-1<\sum_{j=1}^{N} c_{i j} x_{j} \leq\left\{y_{i}\right\}+m_{i} .
$$


Therefore

$$
\begin{aligned}
F(\mathbf{t}, \mathbf{y})= & \left(\prod_{j=1}^{M+N} \frac{t_{j}}{e^{t_{j}}-1}\right) \sum_{m_{1}=c_{1}^{-}}^{c_{1}^{+}} \cdots \sum_{m_{M}=c_{M}^{-}}^{c_{M}^{+}} \exp \left(\sum_{i=1}^{M} t_{N+i}\left(\left\{y_{i}\right\}+m_{i}\right)\right) \\
& \times \int_{\mathcal{P}_{\mathbf{m}, \mathbf{y}}} \exp \left(\sum_{j=1}^{N}\left(a_{j}+b_{j}\right) x_{j}\right) \prod_{j=1}^{N} d x_{j},
\end{aligned}
$$

which is a meromorphic function in $\mathbf{t}$ on the whole space $\mathbb{C}^{M+N}$.

Lemma 3.4. The function $P(\mathbf{k}, \mathbf{y})$ is continuous with respect to $\mathbf{y}$ on $\mathbb{R}^{M}$. The function $F(\mathbf{t}, \mathbf{y})$ is continuous on $\{t \in \mathbb{C}|| t \mid<2 \pi\}^{M+N} \times \mathbb{R}^{M}$ and holomorphic in $\mathbf{t}$ for a fixed $\mathbf{y} \in \mathbb{R}^{M}$.

Proof. For $\mathbf{y} \in \mathbb{R}^{M}$ and $\mathbf{x}=\left(x_{j}\right)_{1 \leq j \leq N} \in \mathbb{R}^{N}$ let $h(\mathbf{y}, \mathbf{x})$ be the integrand of (3.18). Let $\left\{\mathbf{y}_{n}\right\}_{n=1}^{\infty}$ be a sequence in $\mathbb{R}^{M}$ with $\lim _{n \rightarrow \infty} \mathbf{y}_{n}=\mathbf{y}_{\infty}$ and we set $h_{n}(\mathbf{x})=h\left(\mathbf{y}_{n}, \mathbf{x}\right)$ and $h_{\infty}(\mathbf{x})=h\left(\mathbf{y}_{\infty}, \mathbf{x}\right)$. Then for $\mathbf{x} \in[0,1]^{N}$,

$$
\lim _{n \rightarrow \infty} h_{n}(\mathbf{x})=h_{\infty}(\mathbf{x})
$$

holds if $\mathbf{x}$ satisfies

$$
\left(\mathbf{y}_{\infty}\right)_{i}-\sum_{j=1}^{N} c_{i j} x_{j} \notin \mathbb{Z},
$$

for all $1 \leq i \leq M$. Hence (3.34) holds almost everywhere and we have

$$
\begin{aligned}
\lim _{n \rightarrow \infty} P\left(\mathbf{k}, \mathbf{y}_{n}\right) & =\lim _{n \rightarrow \infty} \int_{[0,1]^{N}} h_{n}(\mathbf{x}) \prod_{j=1}^{N} d x_{j} \\
& =\int_{[0,1]^{N}} \lim _{n \rightarrow \infty} h_{n}(\mathbf{x}) \prod_{j=1}^{N} d x_{j} \\
& =\int_{[0,1]^{N}} h_{\infty}(\mathbf{x}) \prod_{j=1}^{N} d x_{j} \\
& =P\left(\mathbf{k}, \mathbf{y}_{\infty}\right),
\end{aligned}
$$

where we have used the uniform boundedness $h_{n}(\mathbf{x}) \leq C$ for some $C>0$ and for all $n \in \mathbb{N}$ and $\mathbf{x} \in[0,1]^{N}$.

Combining the continuity of $P(\mathbf{k}, \mathbf{y})$, definition (3.19) and Lemma 3.3, we obtain the continuity and the holomorphy of $F(\mathbf{t}, \mathbf{y})$.

Now we return to the situation $\mathbf{y} \in V_{I^{c}}$. Let $y_{i}=\left\langle\mathbf{y}, \mu_{i}\right\rangle$ for $i \in I^{c}$ and we identify $\mathbf{y}$ with $\left(y_{i}\right)_{i \in I^{c}} \in \mathbb{R}^{\left|I^{c}\right|}$. We set $\mathbb{Q}[\mathbf{y}]=\mathbb{Q}\left[\left(y_{i}\right)_{i \in I^{c}}\right], A(\mathbf{y})=\sum_{i \in I^{c}} \mathbb{Z} y_{i}+\mathbb{Z}$ and $|\mathbf{k}|=\sum_{\gamma \in D_{I^{c}}} k_{\gamma}$. Let

$$
\bar{D}=\left\{\gamma \circ \tau(-\nu) \mid \gamma \in D_{I^{c}} \backslash B_{I^{c}}\right\}, \quad \nu=\sum_{i \in I^{c}} c_{i} \mu_{i} \in P_{I^{c}}
$$


Theorem 3.5. Assume the same condition as in Theorem 3.1. Moreover we assume that $D_{I^{c}} \subset \widehat{Q}^{\vee}$ and $s_{\gamma}=k_{\gamma}$ are integers for all $\gamma \in D_{I^{c}}$ such that $k_{\gamma} \geq 2$ for $\gamma \in B_{I^{c}}$ and $k_{\gamma} \geq 1$ otherwise. Then $f^{\sharp} \in \mathfrak{F}\left(P_{I}\right)$ in (3.8) is of the form

$$
f^{\sharp}(\lambda)=e^{-2 \pi \sqrt{-1}\langle\mathbf{y}, \nu\rangle} \sum_{k=0}^{|\mathbf{k}|}(\pi \sqrt{-1})^{|\mathbf{k}|-(k+N)} \sum_{\eta} \frac{f_{\eta}^{(k)}(\lambda)}{g_{\eta}^{(k)}(\lambda)},
$$

where $\eta$ runs over a certain finite set of indices, $N=\left|D_{I^{c}} \backslash B_{I^{c}}\right|$ and

$$
f_{\eta}^{(k)} \in \mathbb{Q}[\mathbf{y}] e^{\pi \sqrt{-1} \mathbb{Q} \otimes A(\mathbf{y}) \bar{D}}, \quad g_{\eta}^{(k)} \in(A(\mathbf{y}) \bar{D})^{k+N} .
$$

Proof. From (3.8), we have

$$
f^{\sharp}(\lambda)=(-1)^{\left|D_{I^{c}}\right|} e^{-2 \pi \sqrt{-1}\langle\mathbf{y}, \nu\rangle}\left(\prod_{\gamma \in D_{I^{c}}} \frac{(2 \pi \sqrt{-1})^{k_{\gamma}}}{k_{\gamma} !}\right) P(\mathbf{k}, \mathbf{y}, \lambda),
$$

where for $\mathbf{k}=\left(k_{\gamma}\right)_{\gamma \in D_{I^{c}}}$,

$$
\begin{aligned}
P(\mathbf{k}, \mathbf{y}, \lambda)=\int_{0}^{1} & \ldots \int_{0}^{1} \exp \left(-2 \pi \sqrt{-1} \sum_{\gamma \in D_{I^{c}} \backslash B_{I^{c}}} \gamma(\lambda-\nu) x_{\gamma}\right)\left(\prod_{\gamma \in D_{I^{c} \backslash B_{I^{c}}}} B_{k_{\gamma}}\left(x_{\gamma}\right)\right) \\
& \times\left(\prod_{i \in I^{c}} B_{k_{\gamma_{i}}}\left(\left\{y_{i}-\sum_{\gamma \in D_{I^{c}} \backslash B_{I^{c}}} x_{\gamma}\left\langle\eta_{\gamma}, \mu_{i}\right\rangle\right\}\right)\right) \prod_{\gamma \in D_{I^{c}} \backslash B_{I^{c}}} d x_{\gamma} .
\end{aligned}
$$

Hence $P(\mathbf{k}, \mathbf{y}, \lambda)$ is of the form (3.18) . Therefore, by applying Lemma 3.3 , we find that $P(\mathbf{k}, \mathbf{y}, \lambda)$ is obtained as the coefficient of the term

$$
\prod_{\gamma \in D_{I^{c}}} t_{\gamma}^{k_{\gamma}}
$$

in the generating function

$$
\begin{aligned}
F(\mathbf{t}, \mathbf{y}, \lambda)= & \sum_{\mathbf{k} \in \mathbb{N}_{0}^{\left|D_{I^{c}}\right|}} P(\mathbf{k}, \mathbf{y}, \lambda) \prod_{\gamma \in D_{I^{c}}} \frac{t_{\gamma}^{k_{\gamma}}}{k_{\gamma} !} \\
= & \left(\prod_{\gamma \in D_{I^{c}}} \frac{t_{\gamma}}{e^{t_{\gamma}}-1}\right) \sum_{\substack{m_{i}=0 \\
i \in I^{c}}}^{c_{i}^{+}} \exp \left(\sum_{i \in I^{c}} t_{\gamma_{i}}\left(\left\{y_{i}\right\}+m_{i}\right)\right) \\
& \times \int_{\mathcal{P}_{\mathbf{m}, \mathbf{y}}} \exp ((\mathbf{a}+\mathbf{b}) \cdot \mathbf{x}) \prod_{\gamma \in D_{I^{c} \backslash B_{I^{c}}}} d x_{\gamma},
\end{aligned}
$$

where $\mathbf{a}=\left(a_{\gamma}\right)_{\gamma \in D_{I^{c}} \backslash B_{I^{c}}} \in \mathbb{R}^{N}, \mathbf{b}=\left(b_{\gamma}\right)_{\gamma \in D_{I^{c}} \backslash B_{I^{c}}} \in \mathbb{C}^{N}$ with

$$
\begin{aligned}
& a_{\gamma}=t_{\gamma}-\sum_{i \in I^{c}} t_{\gamma_{i}}\left\langle\eta_{\gamma}, \mu_{i}\right\rangle, \\
& b_{\gamma}=-2 \pi \sqrt{-1} \gamma(\lambda-\nu) .
\end{aligned}
$$

Since $\left\langle\eta_{\gamma}, \mu_{i}\right\rangle \in \mathbb{Z}$, any vertex $\mathbf{p}_{j}$ of $\mathcal{P}_{\mathbf{m}, \mathbf{y}}$ satisfies

$$
\left(\mathbf{p}_{j}\right)_{\gamma} \in \sum_{i \in I^{c}} \mathbb{Q} y_{i}+\mathbb{Q}=\mathbb{Q} \otimes A(\mathbf{y}) .
$$


The first two factors of the last member of (3.43) is expanded as

$$
\left(\prod_{\gamma \in D_{I^{c}}} \frac{t_{\gamma}}{e^{t_{\gamma}}-1}\right)\left(\prod_{i \in I^{c}} \exp \left(t_{\gamma_{i}}\left(\left\{y_{i}\right\}+m_{i}\right)\right)\right)=\sum_{\mathbf{k}^{\prime} \in \mathbb{N}_{0}^{\mid D_{I}}{ }^{c} \mid} P_{\mathbf{k}^{\prime}}(\mathbf{y}) \prod_{\gamma \in D_{I^{c}}} t_{\gamma}^{k_{\gamma}^{\prime}},
$$

where $P_{\mathbf{k}^{\prime}}(\mathbf{y}) \in \mathbb{Q}[\mathbf{y}]$ is of total degree at most $\left|\mathbf{k}^{\prime}\right|$.

Next we calculate the contribution of $\mathbf{t}$ of the integral part. By a triangulation $\mathcal{P}_{\mathbf{m}, \mathbf{y}}=$ $\bigcup_{l=1}^{L(\mathbf{m})} \sigma_{l, \mathbf{m}, \mathbf{y}}$ in Theorem 2.4. the integral on $\mathcal{P}_{\mathbf{m}, \mathbf{y}}$ is reduced to those on $\sigma_{l, \mathbf{m}, \mathbf{y}}$. Since (3.42) is of total degree $|\mathbf{k}|$, and $\mathbf{a}$ is of the same degree as $\mathbf{t}$ by (3.44), the contribution of $\mathbf{t}$ comes from terms of total degree $\kappa \leq|\mathbf{k}|$ with respect to $\mathbf{a}$. By Lemma 2.11, we see that these terms are calculated as the special values of the functions $h\left(\mathbf{b}^{\prime}\right)$ at $\mathbf{b}$, where $h\left(\mathbf{b}^{\prime}\right)$ is a holomorphic function on $\mathbb{C}^{N}$ of the form

$$
\operatorname{Vol}\left(\sigma_{l, \mathbf{m}, \mathbf{y}}\right) \sum_{q=0}^{N} \sum_{\substack{\kappa_{0}, \ldots, \kappa_{N} \geq 0 \\ \kappa_{0}+\cdots+\kappa_{N}=\kappa}} c_{q, \kappa_{0}, \ldots, \kappa_{N}} \frac{e^{\mathbf{b}^{\prime} \cdot \mathbf{p}_{j_{q}}}}{\prod_{\substack{q^{\prime}=0 \\ q^{\prime} \neq q}}^{N}\left(\mathbf{b}^{\prime} \cdot\left(\mathbf{p}_{j_{q}}-\mathbf{p}_{j_{q^{\prime}}}\right)\right)^{\kappa_{q^{\prime}}+1}},
$$

and $\mathbf{p}_{j_{q}}$ 's are the vertices of $\sigma_{l, \mathbf{m}, \mathbf{y}}$ and $\operatorname{Vol}\left(\sigma_{l, \mathbf{m}, \mathbf{y}}\right) \in \mathbb{Q}[\mathbf{y}]$ is of total degree at most $N$ due to (3.46). Since

$$
\mathbf{b} \cdot \mathbf{p}_{j}=-2 \pi \sqrt{-1} \sum_{\gamma \in D_{I^{c}} \backslash B_{I^{c}}} \gamma(\lambda-\nu)\left(\mathbf{p}_{j}\right)_{\gamma} \in \pi \sqrt{-1} \mathbb{Q} \otimes A(\mathbf{y}) \bar{D},
$$

each term of (3.48) is an element of

$$
\mathbb{Q}[\mathbf{y}] \frac{e^{\pi \sqrt{-1} \mathbb{Q} \otimes A(\mathbf{y}) \bar{D}}}{(\pi \sqrt{-1} \mathbb{Q} \otimes A(\mathbf{y}) \bar{D})^{\kappa^{\prime}+N}}=\frac{1}{(\pi \sqrt{-1})^{\kappa^{\prime}+N}} \mathbb{Q}[\mathbf{y}] \frac{e^{\pi \sqrt{-1} \mathbb{Q} \otimes A(\mathbf{y}) \bar{D}}}{(A(\mathbf{y}) \bar{D})^{\kappa^{\prime}+N}},
$$

where $0 \leq \kappa^{\prime} \leq \kappa \leq|\mathbf{k}|$.

Combining (3.47) and (3.48) for all $\mathbf{m}$ and $l \in L(\mathbf{m})$ appearing in the sum, we see that the coefficient of (3.42) is of the form (3.38).

Remark 3.6. It may happen that the denominator of (3.38) vanishes. However the original form (3.8) implies that $f^{\sharp}$ is well-defined on $P_{I}$. In fact, the values can be obtained by use of analytic continuation in (3.48).

Remark 3.7. It should be noted that in Theorems 3.1 and 3.5 we have treated $\mathbf{y} \in V_{I^{c}}$ as a fixed parameter. In general, as a function of $\mathbf{y} \in V_{I^{c}}$, (3.38) is not a real analytic function on the whole space $V_{I^{c}}$. We study this fact in a special case in Section 6 ,

We conclude this section with the following proposition, whose proof is a direct generalization of that of (2.1) in [12].

Proposition 3.8. Let $f, g \in \mathfrak{F}(P)$ and $J \subset\{1, \ldots, r\}$. Assume that $J=J_{1} \amalg J_{2}$ and that $f$ and $g$ are decomposed as

$$
f\left(\lambda_{1}+\lambda_{2}\right)=f_{1}\left(\lambda_{1}\right) f_{2}\left(\lambda_{2}\right), \quad g\left(\lambda_{1}+\lambda_{2}\right)=g_{1}\left(\lambda_{1}\right) g_{2}\left(\lambda_{2}\right),
$$

for $f_{1}, g_{1} \in \mathfrak{F}\left(P_{J_{1}}\right), f_{2}, g_{2} \in \mathfrak{F}\left(P_{J_{2}}\right), \lambda_{1} \in P_{J_{1}}, \lambda_{2} \in P_{J_{2}}$. Then we have

$$
\zeta(f, g ; J ; \Delta)=\zeta\left(f_{1}, g_{1} ; J_{1} ; \Delta\right) \zeta\left(f_{2}, g_{2} ; J_{2} ; \Delta\right) .
$$




\section{Functional Relations, value Relations and Generating Functions}

Hereafter we deal with the special case

$$
\begin{aligned}
& f(\lambda)=e^{2 \pi \sqrt{-1}\langle\mathbf{y}, \lambda\rangle}, \\
& g(\lambda)=\prod_{\alpha \in \Delta_{+}}\left\langle\alpha^{\vee}, \lambda\right\rangle^{s_{\alpha}},
\end{aligned}
$$

for $\mathbf{y} \in V$ and $\mathbf{s}=\left(s_{\alpha}\right)_{\alpha \in \bar{\Delta}} \in \mathbb{C}^{\left|\Delta_{+}\right|}$, where $\bar{\Delta}$ is the quotient of $\Delta$ obtained by identifying $\alpha$ and $-\alpha$. We define an action of $\operatorname{Aut}(\Delta)$ by

$$
(w \mathbf{s})_{\alpha}=s_{w^{-1} \alpha},
$$

and let

$$
\zeta_{r}(\mathbf{s}, \mathbf{y} ; \Delta)=\sum_{\lambda \in P_{++}} e^{2 \pi \sqrt{-1}\langle\mathbf{y}, \lambda\rangle} \prod_{\alpha \in \Delta_{+}} \frac{1}{\left\langle\alpha^{\vee}, \lambda\right\rangle^{s_{\alpha}}}
$$

Then we have

$$
\zeta(f, g ; J ; \Delta)= \begin{cases}\zeta_{r}(\mathbf{s}, \mathbf{y} ; \Delta), & \text { if } J=\{1, \ldots, r\} \\ 0, & \text { otherwise }\end{cases}
$$

because for $J \neq\{1, \ldots, r\}$ we have $P_{J++} \subset H_{\Delta \vee}=H_{g}$. When $\mathbf{y}=0$, the function $\zeta_{r}(\mathbf{s} ; \Delta)=\zeta_{r}(\mathbf{s}, 0 ; \Delta)$ coincides with the zeta-function of the root system $\Delta$, defined by (3.1) of [12]. Therefore (4.4) is the Lerch-type generalization of zeta-functions of root systems. Also we have

$$
S(f, g ; I ; \Delta)=\sum_{\lambda \in \iota^{*-1}\left(P_{I+}\right) \backslash H_{\Delta} \vee} e^{2 \pi \sqrt{-1}\langle\mathbf{y}, \lambda\rangle} \prod_{\alpha \in \Delta_{+}} \frac{1}{\left\langle\alpha^{\vee}, \lambda\right\rangle^{s_{\alpha}}},
$$

which we denote by $S(\mathbf{s}, \mathbf{y} ; I ; \Delta)$. Note that $H_{\Delta} \vee$ coincides with the set of all walls of Weyl chambers. Let

$$
\mathcal{S}=\left\{\mathbf{s}=\left(s_{\alpha}\right) \in \mathbb{C}^{\left|\Delta_{+}\right|} \mid \Re s_{\alpha}>1 \text { for } \alpha \in \Delta_{+}\right\} .
$$

Note that $\mathcal{S}$ is an $\operatorname{Aut}(\Delta)$-invariant set.

Lemma 4.1. $\zeta_{r}(\mathbf{s}, \mathbf{y} ; \Delta)$ and $S(\mathbf{s}, \mathbf{y} ; I ; \Delta)$ are absolutely convergent, uniformly on $\mathcal{D} \times V$ where $\mathcal{D}$ is any compact subset of the set $\mathcal{S}$. Hence they are continuous on $\mathcal{S} \times V$, and holomorphic in $\mathbf{s}$ for a fixed $\mathbf{y} \in V$.

Proof. Since for $\mathbf{s} \in \mathcal{D}, \alpha \in \Delta_{+}$and $\lambda \in P \backslash H_{\Delta^{\vee}}$,

$$
\left|\frac{1}{\left\langle\alpha^{\vee}, \lambda\right\rangle^{s_{\alpha}}}\right| \leq \frac{e^{\pi\left|\Im s_{\alpha}\right|}}{\left|\left\langle\alpha^{\vee}, \lambda\right\rangle\right|^{\Re s_{\alpha}}}
$$

(the factor $e^{\pi\left|\Im s_{\alpha}\right|}$ appears when $\left\langle\alpha^{\vee}, \lambda\right\rangle$ is negative), we have

$$
\left|\prod_{\alpha \in \Delta_{+}} \frac{1}{\left\langle\alpha^{\vee}, \lambda\right\rangle^{s_{\alpha}}}\right| \leq h(\mathbf{s}) \prod_{\alpha \in \Delta_{+}} \frac{1}{\left|\left\langle\alpha^{\vee}, \lambda\right\rangle\right|^{\Re s_{\alpha}}} \leq h(\mathbf{s}) \prod_{\alpha \in \Psi} \frac{1}{\left|\left\langle\alpha^{\vee}, \lambda\right\rangle\right|^{\Re s_{\alpha}}} \leq A \prod_{\alpha \in \Psi} \frac{1}{\left|\left\langle\alpha^{\vee}, \lambda\right\rangle\right|^{B}},
$$

where $h(\mathbf{s})=\prod_{\alpha \in \Delta_{+}} e^{\pi\left|\Im_{s}\right|}$ and $A=\max _{\mathbf{s} \in \mathcal{D}} h(\mathbf{s}), B=\min _{\alpha \in \Psi}\left(\min _{\mathbf{s} \in \mathcal{D}} \Re s_{\alpha}\right)>1$. It follows that

$$
\sum_{\lambda \in P \backslash H_{\Delta^{\vee}}} \prod_{\alpha \in \Psi} \frac{1}{\left|\left\langle\alpha^{\vee}, \lambda\right\rangle\right|^{B}}=|W| \sum_{\lambda \in P_{+}+} \prod_{\alpha \in \Psi} \frac{1}{\left|\left\langle\alpha^{\vee}, \lambda\right\rangle\right|^{B}}=|W|(\zeta(B))^{r}<\infty,
$$


and hence the uniform and absolute convergence on $\mathcal{D} \times V$.

Remark 4.2. Although the statements in Lemma 4.1 and in the rest of this paper hold for larger regions than $\mathcal{S}$, we work with $\mathcal{S}$ for simplicity. For instance, the above proof of Lemma 4.1 holds for the region $\left\{\mathbf{s}=\left(s_{\alpha}\right) \in \mathbb{C}^{\left|\Delta_{+}\right|} \mid \Re s_{\alpha}>1\right.$ for $\alpha \in \Psi, \quad \Re s_{\alpha}>$ 0 otherwise $\}$.

First we apply Theorem 3.1 to the case $I \neq \emptyset$. Then Theorem 3.1 implies the following theorem:

Theorem 4.3. When $I \neq \emptyset$, for $\mathbf{s} \in \mathcal{S}$ and $\mathbf{y} \in V$, we have

$$
\begin{aligned}
& =\sum_{w \in W^{I}}\left(\prod_{\alpha \in \Delta_{w^{-1}}}(-1)^{-s_{\alpha}}\right) \zeta_{r}\left(w^{-1} \mathbf{s}, w^{-1} \mathbf{y} ; \Delta\right) \\
& =(-1)^{\left|\Delta_{+} \backslash \Delta_{I+}\right|}\left(\prod_{\alpha \in \Delta_{+} \backslash \Delta_{I+}} \frac{(2 \pi \sqrt{-1})^{s_{\alpha}}}{\Gamma\left(s_{\alpha}+1\right)}\right) \sum_{\lambda \in P_{I++}} e^{2 \pi \sqrt{-1}\langle\mathbf{y}, \lambda\rangle} \prod_{\alpha \in \Delta_{I+}} \frac{1}{\left\langle\alpha^{\vee}, \lambda\right\rangle^{s_{\alpha}}} \\
& \quad \times \int_{0}^{1} \ldots \int_{0}^{1} \exp \left(-2 \pi \sqrt{-1} \sum_{\alpha \in \Delta_{+} \backslash\left(\Delta_{I+} \cup \Psi\right)} x_{\alpha}\left\langle\alpha^{\vee}, \lambda\right\rangle\right)\left(\prod_{\alpha \in \Delta_{+} \backslash\left(\Delta_{I+} \cup \Psi\right)} L_{s_{\alpha}}\left(x_{\alpha}, 0\right)\right) \\
& \quad \times\left(\prod_{i \in I^{c}} L_{s_{\alpha_{i}}}\left(\left\langle\mathbf{y}, \lambda_{i}\right\rangle-\sum_{\alpha \in \Delta_{+} \backslash\left(\Delta_{I+} \cup \Psi\right)} x_{\alpha}\left\langle\alpha^{\vee}, \lambda_{i}\right\rangle, 0\right)\right) \prod_{\alpha \in \Delta_{+} \backslash\left(\Delta_{I+} \cup \Psi\right)} d x_{\alpha} .
\end{aligned}
$$

The second member consists of $\left(W(\Delta): W\left(\Delta_{I}\right)\right)$ terms.

Proof. It is easy to check (3.4) and (3.5) for (4.1) and (4.2), with $D_{I^{c}}=\Delta_{+} \backslash \Delta_{I+}$, $B_{I^{c}}=\Psi_{I^{c}}$ (hence $c_{\gamma}=0$ for all $\gamma \in D_{I^{c}}$ ), and $g^{\sharp}(\lambda)=\prod_{\alpha \in \Delta_{I+}}\left\langle\alpha^{\vee}, \lambda\right\rangle^{s_{\alpha}}$ for $\lambda \in P_{I+}$. Since $P_{J++} \subset H_{g^{\sharp}}$ for all $J \subsetneq I$, we have the second equality.

Next we check the first equality. From (3.6), (4.5) and Theorem 3.1 (iii), we have

$$
S(\mathbf{s}, \mathbf{y} ; I ; \Delta)=\sum_{w \in W^{I}} \zeta\left(w^{-1} f, w^{-1} g ;\{1, \ldots, r\} ; \Delta\right) .
$$

Further,

$$
\begin{aligned}
\zeta\left(w^{-1} f ; w^{-1} g ;\{1, \ldots, r\} ; \Delta\right) & =\sum_{\lambda \in P_{++}} e^{2 \pi \sqrt{-1}\langle\mathbf{y}, w \lambda\rangle} \prod_{\alpha \in \Delta_{+}} \frac{1}{\left\langle\alpha^{\vee}, w \lambda\right\rangle^{s_{\alpha}}} \\
& =\sum_{\lambda \in P_{++}} e^{2 \pi \sqrt{-1}\left\langle w^{-1} \mathbf{y}, \lambda\right\rangle} \prod_{\alpha \in \Delta_{+}} \frac{1}{\left\langle w^{-1} \alpha^{\vee}, \lambda\right\rangle^{s_{\alpha}}} \\
& =\sum_{\lambda \in P_{++}} e^{2 \pi \sqrt{-1}\left\langle w^{-1} \mathbf{y}, \lambda\right\rangle} \prod_{\alpha \in w^{-1} \Delta_{+}} \frac{1}{\left\langle\alpha^{\vee}, \lambda\right\rangle^{s_{w \alpha}}},
\end{aligned}
$$

by rewriting $\alpha$ as $w \alpha$. When

$$
\alpha \in w^{-1} \Delta_{+} \cap \Delta_{-}=-\left(\Delta_{+} \cap w^{-1} \Delta_{-}\right)=-\Delta_{w},
$$


we further replace $\alpha$ by $-\alpha$. Then we have

$$
\begin{aligned}
\zeta\left(w^{-1} f ; w^{-1} g ;\{1, \ldots, r\} ; \Delta\right) & \\
& =\left(\prod_{\alpha \in \Delta_{w}}(-1)^{-s_{w \alpha}}\right) \sum_{\lambda \in P_{++}} e^{2 \pi \sqrt{-1}\left\langle w^{-1} \mathbf{y}, \lambda\right\rangle} \prod_{\alpha \in \Delta_{+}} \frac{1}{\left\langle\alpha^{\vee}, \lambda\right\rangle^{s_{w \alpha}}} \\
& =\left(\prod_{\alpha \in \Delta_{w^{-1}}}(-1)^{-s_{\alpha}}\right) \zeta_{r}\left(w^{-1} \mathbf{s}, w^{-1} \mathbf{y} ; \Delta\right),
\end{aligned}
$$

where we have used the fact that $w \Delta_{w}=-\Delta_{w^{-1}}$. Hence the first equality follows.

Lemma 2.1 implies that the second member of (4.11) consists of $\left(W(\Delta): W\left(\Delta_{I}\right)\right)$ terms.

Next we deal with the case $I=\emptyset$. Let $S(\mathbf{s}, \mathbf{y} ; \Delta)=S(\mathbf{s}, \mathbf{y} ; \emptyset ; \Delta)$. Then we have the following theorem by Theorem 3.1 .

Theorem 4.4. For $\mathrm{s} \in \mathcal{S}$ and $\mathrm{y} \in V$,

$$
\begin{aligned}
& S(\mathbf{s}, \mathbf{y} ; \Delta)= \sum_{w \in W}\left(\prod_{\alpha \in \Delta_{w^{-1}}}(-1)^{-s_{\alpha}}\right) \zeta_{r}\left(w^{-1} \mathbf{s}, w^{-1} \mathbf{y} ; \Delta\right) \\
&=(-1)^{\left|\Delta_{+}\right|}\left(\prod_{\alpha \in \Delta_{+}} \frac{(2 \pi \sqrt{-1})^{s_{\alpha}}}{\Gamma\left(s_{\alpha}+1\right)}\right) \int_{0}^{1} \ldots \int_{0}^{1}\left(\prod_{\alpha \in \Delta_{+} \backslash \Psi} L_{s_{\alpha}}\left(x_{\alpha}, 0\right)\right) \\
& \times\left(\prod_{i=1}^{r} L_{s_{\alpha_{i}}}\left(\left\langle\mathbf{y}, \lambda_{i}\right\rangle-\sum_{\alpha \in \Delta_{+} \backslash \Psi} x_{\alpha}\left\langle\alpha^{\vee}, \lambda_{i}\right\rangle, 0\right)\right) \prod_{\alpha \in \Delta_{+} \backslash \Psi} d x_{\alpha} .
\end{aligned}
$$

The above two theorems are general functional relations among zeta-functions of root systems with exponential factors. In some cases it is possible to deduce, from these theorems, more explicit functional relations among zeta-functions. (See Example 7.5)). However in general it is not easy to deduce explicit forms of functional relations from (4.11) by direct calculations. Therefore, in our forthcoming paper [14], we will consider some structural background of our technique more deeply and will present much improved versions of Theorem 4.5 and Theorem 6.2. In fact by using these results, we will give explicit forms of other concrete examples which we do not treat in this paper. On the other hand, in [15] we will introduce another technique of deducing explicit forms. This can be regarded as a certain refinement of the " $u$-method" developed in our previous papers. By using this technique, we give explicit functional relations among zeta-functions associated with root systems of types $A_{3}, C_{2}\left(\simeq B_{2}\right), B_{3}$ and $C_{3}$.

Now we study special values of $S(\mathbf{s}, \mathbf{y} ; \Delta)$. We recall that by (3.16)

$$
L_{k}(x, 0)=B_{k}(\{x\})
$$

for a real number $x$. Motivated by this observation, for $\mathbf{k}=\left(k_{\alpha}\right)_{\alpha \in \bar{\Delta}} \in \mathbb{N}_{0}^{\left|\Delta_{+}\right|}$and $\mathbf{y} \in V$ we define

$$
\begin{aligned}
P(\mathbf{k}, \mathbf{y} ; \Delta)=\int_{0}^{1} & \ldots \int_{0}^{1}\left(\prod_{\alpha \in \Delta_{+} \backslash \Psi} B_{k_{\alpha}}\left(x_{\alpha}\right)\right) \\
& \times\left(\prod_{i=1}^{r} B_{k_{\alpha_{i}}}\left(\left\{\left\langle\mathbf{y}, \lambda_{i}\right\rangle-\sum_{\alpha \in \Delta_{+} \backslash \Psi} x_{\alpha}\left\langle\alpha^{\vee}, \lambda_{i}\right\rangle\right\}\right)\right) \prod_{\alpha \in \Delta_{+} \backslash \Psi} d x_{\alpha},
\end{aligned}
$$


so that

$$
S(\mathbf{k}, \mathbf{y} ; \Delta)=(-1)^{\left|\Delta_{+}\right|}\left(\prod_{\alpha \in \Delta_{+}} \frac{(2 \pi \sqrt{-1})^{k_{\alpha}}}{k_{\alpha} !}\right) P(\mathbf{k}, \mathbf{y} ; \Delta)
$$

for $\mathbf{k} \in \mathcal{S} \cap \mathbb{N}_{0}^{\left|\Delta_{+}\right|}$. This function $P(\mathbf{k}, \mathbf{y} ; \Delta)$ may be regarded as a generalization of the Bernoulli periodic functions and $B_{\mathbf{k}}(\Delta)=P(\mathbf{k}, 0 ; \Delta)$ the Bernoulli numbers (see [1]). We define generating functions of $P(\mathbf{k}, \mathbf{y} ; \Delta)$ and $B_{\mathbf{k}}(\Delta)$ as

$$
\begin{gathered}
F(\mathbf{t}, \mathbf{y} ; \Delta)=\sum_{\mathbf{k} \in \mathbb{N}_{0}^{\left|\Delta_{+}\right|}} P(\mathbf{k}, \mathbf{y} ; \Delta) \prod_{\alpha \in \Delta_{+}} \frac{t_{\alpha}^{k_{\alpha}}}{k_{\alpha} !} \\
F(\mathbf{t} ; \Delta)=\sum_{\mathbf{k} \in \mathbb{N}_{0}^{\left|\Delta_{+}\right|}} B_{\mathbf{k}}(\Delta) \prod_{\alpha \in \Delta_{+}} \frac{t_{\alpha}^{k_{\alpha}}}{k_{\alpha} !}
\end{gathered}
$$

where $\mathbf{t}=\left(t_{\alpha}\right)_{\alpha \in \bar{\Delta}}$ with $\left|t_{\alpha}\right|<2 \pi$. Assume $\Delta$ is irreducible and not of type $A_{1}$. Then by Lemma 3.4, we see that $P(\mathbf{k}, \mathbf{y} ; \Delta)$ is continuous in $\mathbf{y}$ on $V$ and $F(\mathbf{t}, \mathbf{y} ; \Delta)$ is continuous on $\{t \in \mathbb{C}|| t \mid<2 \pi\}^{\left|\Delta_{+}\right|} \times V$ and holomorphic in $\mathbf{t}$ for a fixed $\mathbf{y} \in V$. Further by Lemma 3.3 (ii) we see that for a fixed $\mathbf{y} \in V, F(\mathbf{t}, \mathbf{y} ; \Delta)$ is analytically continued to a meromorphic function in $\mathbf{t}$ on the whole space $\mathbb{C}^{\left|\Delta_{+}\right|}$.

Theorem 4.5. We have

$$
\begin{aligned}
F(\mathbf{t}, \mathbf{y} ; \Delta)= & \left.\prod_{\alpha \in \Delta_{+}} \frac{t_{\alpha}}{e^{t_{\alpha}}-1}\right) \int_{0}^{1} \ldots \int_{0}^{1}\left(\prod_{\alpha \in \Delta_{+} \backslash \Psi} \exp \left(t_{\alpha} x_{\alpha}\right)\right) \\
& \times\left(\prod_{i=1}^{r} \exp \left(t_{\alpha_{i}}\left\{\left\langle\mathbf{y}, \lambda_{i}\right\rangle-\sum_{\alpha \in \Delta_{+} \backslash \Psi} x_{\alpha}\left\langle\alpha^{\vee}, \lambda_{i}\right\rangle\right\}\right)\right) \prod_{\alpha \in \Delta_{+} \backslash \Psi} d x_{\alpha} \\
= & \left.\prod_{\alpha \in \Delta_{+}} \frac{t_{\alpha}}{e^{t_{\alpha}}-1}\right)^{2\left\langle\rho^{\vee}, \lambda_{1}\right\rangle-1} \sum_{m_{1}=0}^{2\left\langle\rho^{\vee}, \lambda_{r}\right\rangle-1} \sum_{m_{r}=0}^{r} \exp \left(\sum_{i=1}^{r} t_{\alpha_{i}}\left(\left\{\left\langle\mathbf{y}, \lambda_{i}\right\rangle\right\}+m_{i}\right)\right) \\
& \times \int_{\mathcal{P}_{\mathbf{m}, \mathbf{y}}} \exp \left(\sum_{\alpha \in \Delta_{+} \backslash \Psi} t_{\alpha}^{*} x_{\alpha}\right) \prod_{\alpha \in \Delta_{+} \backslash \Psi} d x_{\alpha},
\end{aligned}
$$

where $\rho^{\vee}=\frac{1}{2} \sum_{\alpha \in \Delta_{+}} \alpha^{\vee}$ is the positive half sum, $t_{\alpha}^{*}=t_{\alpha}-\sum_{i=1}^{r} t_{\alpha_{i}}\left\langle\alpha^{\vee}, \lambda_{i}\right\rangle, \mathbf{m}=$ $\left(m_{1}, \ldots, m_{r}\right)$ and

$$
\mathcal{P}_{\mathbf{m}, \mathbf{y}}=
$$

$$
\left\{\begin{array}{l|l}
\mathbf{x}=\left(x_{\alpha}\right)_{\alpha \in \Delta_{+} \backslash \Psi} & \begin{array}{l}
0 \leq x_{\alpha} \leq 1, \quad\left(\alpha \in \Delta_{+} \backslash \Psi\right) \\
\left\{\left\langle\mathbf{y}, \lambda_{i}\right\rangle\right\}+m_{i}-1 \leq \sum_{\alpha \in \Delta_{+} \backslash \Psi} x_{\alpha}\left\langle\alpha^{\vee}, \lambda_{i}\right\rangle \leq\left\{\left\langle\mathbf{y}, \lambda_{i}\right\rangle\right\}+m_{i}, \quad(1 \leq i \leq r)
\end{array}
\end{array}\right\}
$$

is a convex polytope. In particular, we have

$$
\begin{aligned}
F(\mathbf{t} ; \Delta)= & \left(\prod_{\alpha \in \Delta_{+}} \frac{t_{\alpha}}{e^{t_{\alpha}}-1}\right)^{2\left\langle\rho^{\vee}, \lambda_{1}\right\rangle-1} \sum_{m_{1}=1}^{2\left\langle\rho^{\vee}, \lambda_{r}\right\rangle-1} \operatorname{\sum _{m_{r}=1}^{r}} \exp \left(\sum_{i=1}^{r} t_{\alpha_{i}} m_{i}\right) \\
& \times \int_{\mathcal{P}_{\mathbf{m}}} \exp \left(\sum_{\alpha \in \Delta_{+} \backslash \Psi} t_{\alpha}^{*} x_{\alpha}\right) \prod_{\alpha \in \Delta_{+} \backslash \Psi} d x_{\alpha},
\end{aligned}
$$


where

$$
\begin{aligned}
\mathcal{P}_{\mathbf{m}}= & \mathcal{P}_{\mathbf{m}, 0}= \\
\left\{\mathbf{x}=\left(x_{\alpha}\right)_{\alpha \in \Delta_{+} \backslash \Psi}\right. & \left.\begin{array}{l}
0 \leq x_{\alpha} \leq 1, \quad\left(\alpha \in \Delta_{+} \backslash \Psi\right) \\
m_{i}-1 \leq \sum_{\alpha \in \Delta_{+} \backslash \Psi} x_{\alpha}\left\langle\alpha^{\vee}, \lambda_{i}\right\rangle \leq m_{i}, \quad(1 \leq i \leq r)
\end{array}\right\} .
\end{aligned}
$$

Proof. Applying Lemma 3.3 to the case $N=\left|\Delta_{+} \backslash \Psi\right|, M=|\Psi|, b_{j}=0, k_{j}=k_{\alpha}$, $y_{i}=\left\langle\mathbf{y}, \lambda_{i}\right\rangle$ and $c_{i j}=\left\langle\alpha^{\vee}, \lambda_{i}\right\rangle$, we obtain (4.22).

From the above theorem we can deduce the following formula. In the case when all $k_{\alpha}$ 's are the same, this formula gives a refinement of Witten's formula (1.2). In other words, it gives a multiple generalization of the classical formula (1.3). In [35], Witten showed that the volume of certain moduli spaces can be written in terms of special values of series (1.1). Moreover he remarked that the volume is rational in the orientable case, which implies (1.2). Zagier [36] gives a brief sketch of a more number-theoretic demonstration of (1.2). Szenes [28] provides an algorithm of the evaluations by use of iterated residues. In our method, the rational number $C_{W}(2 k, \mathfrak{g})$ is expressed in terms of generalized Bernoulli numbers, which can be calculated by use of the generating functions.

Theorem 4.6. Assume that $\Delta$ is an irreducible root system. Let $k_{\alpha}=k_{\|\alpha\|} \in \mathbb{N}$ and $\mathbf{k}=\left(k_{\alpha}\right)_{\alpha \in \bar{\Delta}}$. Then we have

$$
\zeta_{r}(2 \mathbf{k} ; \Delta)=\frac{(-1)^{\left|\Delta_{+}\right|}}{|W|}\left(\prod_{\alpha \in \Delta_{+}} \frac{(2 \pi \sqrt{-1})^{2 k_{\alpha}}}{\left(2 k_{\alpha}\right) !}\right) B_{2 \mathbf{k}}(\Delta) \in \mathbb{Q} \pi^{2 \sum_{l} k_{l}\left|\left(\Delta_{+}\right)_{l}\right|},
$$

where $l$ runs over the lengths of roots and $\left(\Delta_{+}\right)_{l}=\left\{\alpha \in \Delta_{+} \mid\|\alpha\|=l\right\}$.

Proof. Since the vertices $\mathbf{p}_{j}$ of $\mathcal{P}_{\mathbf{m}}$ satisfy $\left(\mathbf{p}_{j}\right)_{\alpha} \in \mathbb{Q}$, by Theorem 4.5 and Lemma 2.10, we have

$$
B_{2 \mathbf{k}}(\Delta)=P(2 \mathbf{k}, 0 ; \Delta) \in \mathbb{Q}
$$

and hence by (4.19),

$$
S(2 \mathbf{k}, 0 ; \Delta)=(-1)^{\left|\Delta_{+}\right|}\left(\prod_{\alpha \in \Delta_{+}} \frac{(2 \pi \sqrt{-1})^{2 k_{\alpha}}}{\left(2 k_{\alpha}\right) !}\right) B_{2 \mathbf{k}}(\Delta) \in \mathbb{Q} \pi^{2 \sum_{l} k_{l}\left|\left(\Delta_{+}\right)_{l}\right|} .
$$

On the other hand, by Theorem 4.4

$$
S(2 \mathbf{k}, 0 ; \Delta)=|W| \zeta_{r}(2 \mathbf{k} ; \Delta),
$$

since roots of the same length form a single orbit. Therefore we have (4.26).

Remark 4.7. The assumption of the irreducibility of $\Delta$ in Theorem 4.6 is not essential. Since a reducible root system is decomposed into a direct sum of some irreducible root systems, this assumption can be removed by use of Proposition 3.8 .

Remark 4.8. It is also to be stressed that our formula covers the case when some of the $k_{\alpha}$ 's are not the same. For example, let $X_{r}=C_{2}\left(\simeq B_{2}\right)$. Then we can take the positive roots as $\left\{\alpha_{1}, \alpha_{2}, 2 \alpha_{1}+\alpha_{2}, \alpha_{1}+\alpha_{2}\right\}$ with $\left\|\alpha_{1}^{\vee}\right\|=\left\|\alpha_{1}^{\vee}+2 \alpha_{2}^{\vee}\right\|,\left\|\alpha_{2}^{\vee}\right\|=\left\|\alpha_{1}^{\vee}+\alpha_{2}^{\vee}\right\|$. We see 
that

$$
\begin{aligned}
\zeta_{2}\left(2,4,4,2 ; C_{2}\right) & =\sum_{m=1}^{\infty} \sum_{n=1}^{\infty} \frac{1}{m^{2} n^{4}(m+n)^{4}(m+2 n)^{2}} \\
& =\frac{53}{6810804000} \pi^{12} .
\end{aligned}
$$

Explicit forms of generating functions can be calculated with the aid of Theorem 2.4 and Lemma 2.9. We give some more explicit examples in Section 7.

\section{Actions of extended Weyl groups}

In this section, we study the action of $\widehat{W}$ on $S(\mathbf{s}, \mathbf{y} ; \Delta), F(\mathbf{t}, \mathbf{y} ; \Delta)$ and $P(\mathbf{k}, \mathbf{y} ; \Delta)$. First consider the action of $\operatorname{Aut}(\Delta) \subset \widehat{W}$. Note that $P \backslash H_{\Delta} \vee$ is an $\operatorname{Aut}(\Delta)$-invariant set, because $H_{\Delta} \vee$ is $\operatorname{Aut}(\Delta)$-invariant. An action of $\operatorname{Aut}(\Delta)$ is naturally induced on any function $f$ in $\mathbf{s}$ and $\mathbf{y}$ as follows: For $w \in \operatorname{Aut}(\Delta)$,

$$
(w f)(\mathbf{s}, \mathbf{y})=f\left(w^{-1} \mathbf{s}, w^{-1} \mathbf{y}\right) .
$$

Theorem 5.1. For $\mathbf{s} \in \mathcal{S}$ and $\mathbf{y} \in V$, and for $w \in \operatorname{Aut}(\Delta)$, we have

$$
(w S)(\mathbf{s}, \mathbf{y} ; \Delta)=\left(\prod_{\alpha \in \Delta_{w^{-1}}}(-1)^{-s_{\alpha}}\right) S(\mathbf{s}, \mathbf{y} ; \Delta)
$$

if $s_{\alpha} \in \mathbb{Z}$ for $\alpha \in \Delta_{w^{-1}}$.

Proof. From (4.6), we have

$$
(w S)(\mathbf{s}, \mathbf{y} ; \Delta)=\sum_{\lambda \in P \backslash H_{\Delta^{\vee}}} e^{2 \pi \sqrt{-1}\left\langle w^{-1} \mathbf{y}, \lambda\right\rangle} \prod_{\alpha \in \Delta_{+}} \frac{1}{\left\langle\alpha^{\vee}, \lambda\right\rangle^{s_{w \alpha}}} .
$$

Rewriting $\lambda$ as $w^{-1} \lambda$ and noting that $P \backslash H_{\Delta} \vee$ is $\operatorname{Aut}(\Delta)$-invariant, we have

$$
\begin{aligned}
(w S)(\mathbf{s}, \mathbf{y} ; \Delta) & =\sum_{\lambda \in P \backslash H_{\Delta} \vee} e^{2 \pi \sqrt{-1}\langle\mathbf{y}, \lambda\rangle} \prod_{\alpha \in \Delta_{+}} \frac{1}{\left\langle w \alpha^{\vee}, \lambda\right\rangle^{s_{w \alpha}}} \\
& =\sum_{\lambda \in P \backslash H_{\Delta} \vee} e^{2 \pi \sqrt{-1}\langle\mathbf{y}, \lambda\rangle} \prod_{w^{-1} \alpha \in \Delta_{+}} \frac{1}{\left\langle\alpha^{\vee}, \lambda\right\rangle^{s_{\alpha}}} \\
& =\left(\prod_{\alpha \in \Delta_{w^{-1}}}(-1)^{-s_{\alpha}}\right) S(\mathbf{s}, \mathbf{y} ; \Delta) .
\end{aligned}
$$

Thus we have

Theorem 5.2. For $\mathbf{s} \in \mathcal{S}$ and $\mathbf{y} \in V$, we have $S(\mathbf{s}, \mathbf{y} ; \Delta)=0$ if there exists an element $w \in \operatorname{Aut}(\Delta)_{\mathbf{s}} \cap \operatorname{Aut}(\Delta)_{\mathbf{y}}$ such that $s_{\alpha} \in \mathbb{Z}$ for $\alpha \in \Delta_{w^{-1}}$ and

$$
\sum_{\alpha \in \Delta_{w-1}} s_{\alpha} \notin 2 \mathbb{Z}
$$

where $\operatorname{Aut}(\Delta)_{\mathbf{s}}$ and $\operatorname{Aut}(\Delta)_{\mathbf{y}}$ are the stabilizers of $\mathbf{s}$ and $\mathbf{y}$ respectively by regarding $\mathbf{y} \in$ $V / Q^{\vee}$. 
Proof. Assume (5.5). Then by Theorem 5.1

$$
\left(1-\left(\prod_{\alpha \in \Delta_{w^{-1}}}(-1)^{-s_{\alpha}}\right)\right) S(\mathbf{s}, \mathbf{y} ; \Delta)=0,
$$

which implies $S(\mathbf{s}, \mathbf{y} ; \Delta)=0$.

Lemma 5.3. The extended Weyl group $\operatorname{Aut}(\Delta)$ acts on $\mathbb{C}^{\left|\Delta_{+}\right|}$by

$$
(w \mathbf{t})_{\alpha}:= \begin{cases}t_{w^{-1} \alpha}, & \text { if } \alpha \in \Delta_{+} \backslash \Delta_{w^{-1}}, \\ -t_{w^{-1} \alpha}, & \text { if } \alpha \in \Delta_{w^{-1}},\end{cases}
$$

where $\mathbf{t}=\left(t_{\alpha}\right)_{\alpha \in \bar{\Delta}} \in \mathbb{C}^{\left|\Delta_{+}\right|}$and the representative $\alpha$ runs over $\Delta_{+}$.

Proof. What we have to check is that the definition (5.7) indeed defines an action. Since

$$
(v(w \mathbf{t}))_{\alpha}= \begin{cases}(w \mathbf{t})_{v^{-1} \alpha}, & \text { if } \alpha \in \Delta_{+} \backslash \Delta_{v^{-1}}, \\ -(w \mathbf{t})_{v^{-1} \alpha}, & \text { if } \alpha \in \Delta_{v^{-1}},\end{cases}
$$

we have

$$
(v(w \mathbf{t}))_{\alpha}=t_{(v w)^{-1} \alpha}
$$

if and only if either

(1) $\alpha \in \Delta_{+} \backslash \Delta_{v^{-1}}$ and $v^{-1} \alpha \in \Delta_{+} \backslash \Delta_{w^{-1}}$

or

(2) $\alpha \in \Delta_{v^{-1}}$ and $-v^{-1} \alpha \in \Delta_{w^{-1}}$

holds. Here, the minus sign in the second case is caused by the fact that if $\alpha \in \Delta_{v^{-1}}$ then $v^{-1} \alpha \in v^{-1} \Delta_{v^{-1}}=-\Delta_{v} \subset \Delta_{-}$. Therefore (5.9) is valid if and only if $\alpha \in \Delta_{+}$and

$$
\begin{aligned}
\alpha & \in\left(v\left(\Delta_{+} \backslash \Delta_{w^{-1}}\right) \cap v \Delta_{+}\right) \cup\left(v\left(-\Delta_{w^{-1}}\right) \cap v \Delta_{-}\right) \\
& =\left(v \Delta_{+} \backslash v w \Delta_{-}\right) \cup\left(v \Delta_{-} \cap v w \Delta_{+}\right) \\
& =\left(v \Delta_{+} \cap v w \Delta_{+}\right) \cup\left(v \Delta_{-} \cap v w \Delta_{+}\right) \\
& =v w \Delta_{+} .
\end{aligned}
$$

This condition is equivalent to $\alpha \in \Delta_{+} \backslash \Delta_{(v w)^{-1}}$. This implies $v(w \mathbf{t})=(v w)(\mathbf{t})$.

Note that we defined two types of actions of $\operatorname{Aut}(\Delta)$ on $\mathbb{C}^{\left|\Delta_{+}\right|}$, that is, (4.3) and (5.7). The action (5.7) is used only on the variable $\mathbf{t}$ and should not be confused with the action (4.3).

If $\Delta$ is of type $A_{1}$, then $F\left(\mathbf{t}, \mathbf{y} ; A_{1}\right)=t e^{t\{y\}} /\left(e^{t}-1\right)$ (see Example 7.1 below) is an even or, in other words, Aut $(\Delta)$-invariant function except for $y \in \mathbb{Z}$. In the multiple cases, $F(\mathbf{t}, \mathbf{y} ; \Delta)$ is revealed to be really an $\operatorname{Aut}(\Delta)$-invariant function. To show it, we need some notation and facts. Fix $1 \leq m \leq r$. Note that $\sigma_{m} \Delta_{+}=\left(\Delta_{+} \backslash\left\{\alpha_{m}\right\}\right) \amalg\left\{-\alpha_{m}\right\}$. Let $\Delta_{1}=\left(\Delta_{+} \backslash \Psi\right) \cap \sigma_{m}\left(\Delta_{+} \backslash \Psi\right)$ and $\Psi_{1}=\Psi \cap \sigma_{m}\left(\Delta_{+} \backslash \Psi\right)$ so that $\sigma_{m}\left(\Delta_{+} \backslash \Psi\right)=\Delta_{1} \amalg \Psi_{1}$. Let $\Delta_{2}=\left(\Delta_{+} \backslash \Psi\right) \cap \sigma_{m} \Psi$ and $\Psi_{2}=\Psi \cap \sigma_{m} \Psi$. Then we have $\Delta_{+} \backslash \Psi=\Delta_{1} \amalg \Delta_{2}$ and $\Psi=\Psi_{1} \coprod \Psi_{2} \coprod\left\{\alpha_{m}\right\}$. Moreover we see that $\sigma_{m}$ fixes $\Psi_{2}$ pointwise and $\Psi_{1}=\sigma_{m} \Delta_{2}$.

\section{Lemma 5.4 .}

$$
\sum_{\alpha_{i} \in \Psi_{1}} \lambda_{i}\left\langle\alpha_{i}^{\vee}, \alpha_{m}\right\rangle=\alpha_{m}-2 \lambda_{m}
$$


Proof. Note that $\alpha_{i} \in \Psi_{1}$ if and only if $\left\langle\alpha_{i}^{\vee}, \alpha_{m}\right\rangle \neq 0$ and $\alpha_{i} \neq \alpha_{m}$. Let $v$ be the left-hand side. Then we have

$$
\left\langle\alpha_{k}^{\vee}, v\right\rangle= \begin{cases}\left\langle\alpha_{k}^{\vee}, \alpha_{m}\right\rangle, & \text { if } \alpha_{k} \in \Psi_{1}, \\ 0, & \text { if } \alpha_{k} \in \Psi_{2} \cup\left\{\alpha_{m}\right\},\end{cases}
$$

which determines the right-hand side uniquely.

An action of $\operatorname{Aut}(\Delta)$ is naturally induced on any function $f$ in $\mathbf{t}$ and $\mathbf{y}$ as follows: For $w \in \operatorname{Aut}(\Delta)$,

$$
(w f)(\mathbf{t}, \mathbf{y})=f\left(w^{-1} \mathbf{t}, w^{-1} \mathbf{y}\right) .
$$

Theorem 5.5. Assume that $\Delta$ is an irreducible root system. If $\Delta$ is not of type $A_{1}$, then

$$
(w F)(\mathbf{t}, \mathbf{y} ; \Delta)=F(\mathbf{t}, \mathbf{y} ; \Delta)
$$

for $\mathbf{t} \in \mathbb{C}^{\left|\Delta_{+}\right|}$and $\mathbf{y} \in V$, and for $w \in \operatorname{Aut}(\Delta)$. Hence for $\mathbf{k} \in \mathbb{N}_{0}^{\left|\Delta_{+}\right|}$and $\mathbf{y} \in V$,

$$
(w P)(\mathbf{k}, \mathbf{y} ; \Delta)=\left(\prod_{\alpha \in \Delta_{w^{-1}}}(-1)^{-k_{\alpha}}\right) P(\mathbf{k}, \mathbf{y} ; \Delta) .
$$

Remark 5.6. If $\mathbf{k}$ is in the region $\mathcal{S}$ of absolute convergence with respect to $\mathbf{s}$, the relation (4.19) and Theorem 5.1 immediately imply (5.15), while $\mathbf{k} \notin \mathcal{S}$, it should be proved independently.

Remark 5.7. The assumption of the irreducibility is not essential by the same reason as in Remark 4.7.

Proof. It is sufficient to show (5.14) for the cases $w=\sigma_{m} \in W$ and $w=\omega \in \Omega$ because $\operatorname{Aut}(\Delta)$ is generated by simple reflections and the subgroup $\Omega$. Applying the simple reflection $\sigma_{m}$ to the second member of (4.22), we have

$$
\begin{aligned}
\left(\sigma_{m} F\right)(\mathbf{t}, \mathbf{y} ; \Delta) & \left(\prod_{\alpha \in \Delta_{+}} \frac{t_{\alpha}}{e^{t_{\alpha}}-1}\right) \int_{0}^{1} \ldots \int_{0}^{1}\left(\prod_{\alpha \in \Delta_{+} \backslash \Psi} \exp \left(t_{\sigma_{m} \alpha} x_{\alpha}\right)\right) \\
& \times \exp \left(t_{\alpha_{m}}\left(1-\left\{\left\langle\sigma_{m} \mathbf{y}, \lambda_{m}\right\rangle-\sum_{\alpha \in \Delta_{+} \backslash \Psi} x_{\alpha}\left\langle\alpha^{\vee}, \lambda_{m}\right\rangle\right\}\right)\right) \\
& \times\left(\prod_{\substack{i=1 \\
i \neq m}}^{r} \exp \left(t_{\sigma_{m} \alpha_{i}}\left\{\left\langle\sigma_{m} \mathbf{y}, \lambda_{i}\right\rangle-\sum_{\alpha \in \Delta_{+} \backslash \Psi} x_{\alpha}\left\langle\alpha^{\vee}, \lambda_{i}\right\rangle\right\}\right)\right) \prod_{\alpha \in \Delta_{+} \backslash \Psi} d x_{\alpha},
\end{aligned}
$$

where we have used the fact that by the action of $\sigma_{m}$, the factor $\prod_{\alpha \in \Delta_{+}} t_{\alpha} /\left(e^{t_{\alpha}}-1\right)$ is sent to

$$
\begin{aligned}
\frac{-t_{\sigma_{m} \alpha_{m}}}{e^{-t_{\sigma_{m} \alpha_{m}}}-1} \prod_{\alpha \in \Delta_{+} \backslash\left\{\alpha_{m}\right\}} \frac{t_{\sigma_{m} \alpha}}{e^{t_{\sigma_{m} \alpha}}-1} & =\frac{t_{\sigma_{m} \alpha_{m}} e^{t_{\sigma_{m} \alpha_{m}}}}{e^{t_{\sigma_{m} \alpha_{m}}}-1} \prod_{\alpha \in \Delta_{+} \backslash\left\{\alpha_{m}\right\}} \frac{t_{\sigma_{m} \alpha}}{e^{t_{\sigma_{m} \alpha}}-1} \\
& =e^{t_{\alpha_{m}}} \prod_{\alpha \in \Delta_{+}} \frac{t_{\alpha}}{e^{t_{\alpha}}-1} .
\end{aligned}
$$


Therefore, rewriting $x_{\alpha}$ as $x_{\sigma_{m} \alpha}$, we have

$$
\begin{aligned}
& \left(\sigma_{m} F\right)(\mathbf{t}, \mathbf{y} ; \Delta) \\
& =\left(\prod_{\alpha \in \Delta_{+}} \frac{t_{\alpha}}{e^{t_{\alpha}}-1}\right) \int_{0}^{1} \cdots \int_{0}^{1}\left(\prod_{\alpha \in \Delta_{+} \backslash \Psi} \exp \left(t_{\sigma_{m} \alpha} x_{\sigma_{m} \alpha}\right)\right) \\
& \times \exp \left(t_{\alpha_{m}}\left(1-\left\{\left\langle\mathbf{y}, \sigma_{m} \lambda_{m}\right\rangle-\sum_{\alpha \in \Delta_{+} \backslash \Psi} x_{\sigma_{m} \alpha}\left\langle\sigma_{m} \alpha^{\vee}, \sigma_{m} \lambda_{m}\right\rangle\right\}\right)\right) \\
& \times\left(\prod_{\substack{i=1 \\
i \neq m}}^{r} \exp \left(t_{\sigma_{m} \alpha_{i}}\left\{\left\langle\mathbf{y}, \sigma_{m} \lambda_{i}\right\rangle-\sum_{\alpha \in \Delta_{+} \backslash \Psi} x_{\sigma_{m} \alpha}\left\langle\sigma_{m} \alpha^{\vee}, \sigma_{m} \lambda_{i}\right\rangle\right\}\right)\right) \prod_{\alpha \in \Delta_{+} \backslash \Psi} d x_{\sigma_{m} \alpha} \\
& =\left(\prod_{\alpha \in \Delta_{+}} \frac{t_{\alpha}}{e^{t_{\alpha}}-1}\right) \int_{0}^{1} \cdots \int_{0}^{1}\left(\prod_{\alpha \in \sigma_{m}\left(\Delta_{+} \backslash \Psi\right)} \exp \left(t_{\alpha} x_{\alpha}\right)\right) \\
& \times \exp \left(t_{\alpha_{m}}\left(1-\left\{\left\langle\mathbf{y}, \lambda_{m}-\alpha_{m}\right\rangle-\sum_{\alpha \in \sigma_{m}\left(\Delta_{+} \backslash \Psi\right)} x_{\alpha}\left\langle\alpha^{\vee}, \lambda_{m}-\alpha_{m}\right\rangle\right\}\right)\right) \\
& \times\left(\prod_{\alpha_{i} \in \Psi \backslash\left\{\alpha_{m}\right\}} \exp \left(t_{\sigma_{m} \alpha_{i}}\left\{\left\langle\mathbf{y}, \lambda_{i}\right\rangle-\sum_{\alpha \in \sigma_{m}\left(\Delta_{+} \backslash \Psi\right)} x_{\alpha}\left\langle\alpha^{\vee}, \lambda_{i}\right\rangle\right\}\right)\right) \prod_{\alpha \in \sigma_{m}\left(\Delta_{+} \backslash \Psi\right)} d x_{\alpha} \\
& =\left(\prod_{\alpha \in \Delta_{+}} \frac{t_{\alpha}}{e^{t_{\alpha}}-1}\right) \int_{0}^{1} \ldots \int_{0}^{1}\left(\prod_{\alpha \in \Delta_{1}} \exp \left(t_{\alpha}\left\{x_{\alpha}\right\}\right)\right)\left(\prod_{\alpha_{i} \in \Psi_{1}} \exp \left(t_{\alpha_{i}}\left\{x_{\alpha_{i}}\right\}\right)\right) \\
& \times \exp \left(t_{\alpha_{m}}\left(1-\left\{\left\langle\mathbf{y}, \lambda_{m}-\alpha_{m}\right\rangle-\sum_{\alpha \in \Delta_{1} \cup \Psi_{1}} x_{\alpha}\left\langle\alpha^{\vee}, \lambda_{m}-\alpha_{m}\right\rangle\right\}\right)\right) \\
& \times\left(\prod_{\alpha_{j} \in \Psi_{2}} \exp \left(t_{\alpha_{j}}\left\{\left\langle\mathbf{y}, \lambda_{j}\right\rangle-\sum_{\alpha \in \Delta_{1} \cup \Psi_{1}} x_{\alpha}\left\langle\alpha^{\vee}, \lambda_{j}\right\rangle\right\}\right)\right) \\
& \times\left(\prod_{\alpha_{i} \in \Psi_{1}} \exp \left(t_{\sigma_{m} \alpha_{i}}\left\{\left\langle\mathbf{y}, \lambda_{i}\right\rangle-\sum_{\alpha \in \Delta_{1} \cup \Psi_{1}} x_{\alpha}\left\langle\alpha^{\vee}, \lambda_{i}\right\rangle\right\}\right)\right) \prod_{\alpha \in \Delta_{1} \cup \Psi_{1}} d x_{\alpha} .
\end{aligned}
$$

Here we change variables from $\mathbf{x}=\left(x_{\alpha}\right)_{\alpha \in \Delta_{1} \cup \Psi_{1}}$ to $\mathbf{z}=\left(z_{\alpha}\right)_{\alpha \in \Delta_{1} \cup \Delta_{2}}$ as

$$
z_{\alpha}= \begin{cases}x_{\alpha}, & \text { if } \alpha \in \Delta_{1}, \\ \left\langle\mathbf{y}, \lambda_{i}\right\rangle-\sum_{\beta \in \Delta_{1} \cup \Psi_{1}} x_{\beta}\left\langle\beta^{\vee}, \lambda_{i}\right\rangle, & \text { if } \alpha=\sigma_{m} \alpha_{i} \in \Delta_{2},\end{cases}
$$

so that the Jacobian matrix is calculated as

$$
\frac{\partial \mathbf{z}}{\partial \mathbf{x}}=\left(\begin{array}{cc}
I_{\left|\Delta_{1}\right|} & 0 \\
* & -I_{\left|\Delta_{2}\right|}
\end{array}\right)
$$

where $I_{p}$ is the $p \times p$ identity matrix, since

$$
\begin{aligned}
z_{\sigma_{m} \alpha_{i}} & =\left\langle\mathbf{y}, \lambda_{i}\right\rangle-\sum_{\alpha \in \Delta_{1} \cup \Psi_{1}} x_{\alpha}\left\langle\alpha^{\vee}, \lambda_{i}\right\rangle \\
& =\left\langle\mathbf{y}, \lambda_{i}\right\rangle-\sum_{\alpha \in \Delta_{1}} x_{\alpha}\left\langle\alpha^{\vee}, \lambda_{i}\right\rangle-x_{\alpha_{i}} .
\end{aligned}
$$

Thus we have $|\operatorname{det} \partial \mathbf{x} / \partial \mathbf{z}|=1$. For $\alpha=\sigma_{m} \alpha_{k} \in \Delta_{2}$ and $\alpha_{i} \in \Psi_{1}$, we have

$$
\left\langle\alpha^{\vee}, \lambda_{i}\right\rangle=\left\langle\sigma_{m} \alpha_{k}^{\vee}, \lambda_{i}\right\rangle=\left\langle\alpha_{k}^{\vee}, \sigma_{m} \lambda_{i}\right\rangle=\left\langle\alpha_{k}^{\vee}, \lambda_{i}\right\rangle=\delta_{k i},
$$


and hence

$$
\begin{aligned}
x_{\alpha_{i}} & =\left\langle\mathbf{y}, \lambda_{i}\right\rangle-\sum_{\alpha \in \Delta_{1}} z_{\alpha}\left\langle\alpha^{\vee}, \lambda_{i}\right\rangle-z_{\sigma_{m} \alpha_{i}} \\
& =\left\langle\mathbf{y}, \lambda_{i}\right\rangle-\sum_{\alpha \in \Delta_{+} \backslash \Psi} z_{\alpha}\left\langle\alpha^{\vee}, \lambda_{i}\right\rangle .
\end{aligned}
$$

For the fourth factor of the last integral in (5.18), we have

$$
\left\langle\alpha^{\vee}, \lambda_{j}\right\rangle=\left\langle\sigma_{m} \alpha_{k}^{\vee}, \lambda_{j}\right\rangle=\left\langle\alpha_{k}^{\vee}, \sigma_{m} \lambda_{j}\right\rangle=\left\langle\alpha_{k}^{\vee}, \lambda_{j}\right\rangle=0,
$$

for $\alpha=\sigma_{m} \alpha_{k} \in \Delta_{2}$ and $\alpha_{j} \in \Psi_{2}$, and hence

$$
\begin{aligned}
\left\langle\mathbf{y}, \lambda_{j}\right\rangle-\sum_{\alpha \in \Delta_{1} \cup \Psi_{1}} x_{\alpha}\left\langle\alpha^{\vee}, \lambda_{j}\right\rangle & =\left\langle\mathbf{y}, \lambda_{j}\right\rangle-\sum_{\alpha \in \Delta_{1}} z_{\alpha}\left\langle\alpha^{\vee}, \lambda_{j}\right\rangle \\
& =\left\langle\mathbf{y}, \lambda_{j}\right\rangle-\sum_{\alpha \in \Delta_{+} \backslash \Psi} z_{\alpha}\left\langle\alpha^{\vee}, \lambda_{j}\right\rangle .
\end{aligned}
$$

For the third factor, we have

$$
\begin{aligned}
\left\langle\mathbf{y}, \lambda_{m}-\alpha_{m}\right\rangle- & \sum_{\alpha \in \Delta_{1} \cup \Psi_{1}} x_{\alpha}\left\langle\alpha^{\vee}, \lambda_{m}-\alpha_{m}\right\rangle \\
= & \left\langle\mathbf{y}, \lambda_{m}-\alpha_{m}\right\rangle-\sum_{\alpha \in \Delta_{1}} x_{\alpha}\left\langle\alpha^{\vee}, \lambda_{m}-\alpha_{m}\right\rangle-\sum_{\alpha_{i} \in \Psi_{1}} x_{\alpha_{i}}\left\langle\alpha_{i}^{\vee}, \lambda_{m}-\alpha_{m}\right\rangle \\
= & \left\langle\mathbf{y}, \lambda_{m}-\alpha_{m}\right\rangle-\sum_{\alpha \in \Delta_{1}} x_{\alpha}\left\langle\alpha^{\vee}, \lambda_{m}-\alpha_{m}\right\rangle+\sum_{\alpha_{i} \in \Psi_{1}} x_{\alpha_{i}}\left\langle\alpha_{i}^{\vee}, \alpha_{m}\right\rangle \\
= & \left\langle\mathbf{y}, \lambda_{m}-\alpha_{m}\right\rangle-\sum_{\alpha \in \Delta_{1}} z_{\alpha}\left\langle\alpha^{\vee}, \lambda_{m}-\alpha_{m}\right\rangle \\
& \quad+\sum_{\alpha_{i} \in \Psi_{1}}\left(\left\langle\mathbf{y}, \lambda_{i}\right\rangle-\sum_{\alpha \in \Delta_{+} \backslash \Psi} z_{\alpha}\left\langle\alpha^{\vee}, \lambda_{i}\right\rangle\right)\left\langle\alpha_{i}^{\vee}, \alpha_{m}\right\rangle
\end{aligned}
$$

by using (5.23). Hence we have

$$
\begin{aligned}
& \left\langle\mathbf{y}, \lambda_{m}-\alpha_{m}\right\rangle-\sum_{\alpha \in \Delta_{1} \cup \Psi_{1}} x_{\alpha}\left\langle\alpha^{\vee}, \lambda_{m}-\alpha_{m}\right\rangle \\
& =\left\langle\mathbf{y}, \lambda_{m}-\alpha_{m}\right\rangle-\sum_{\alpha \in \Delta_{1}} z_{\alpha}\left\langle\alpha^{\vee}, \lambda_{m}-\alpha_{m}\right\rangle \\
& \quad+\sum_{\alpha_{i} \in \Psi_{1}}\left\langle\mathbf{y}, \lambda_{i}\right\rangle\left\langle\alpha_{i}^{\vee}, \alpha_{m}\right\rangle-\sum_{\alpha \in \Delta_{+} \backslash \Psi} \sum_{\alpha_{i} \in \Psi_{1}} z_{\alpha}\left\langle\alpha^{\vee}, \lambda_{i}\right\rangle\left\langle\alpha_{i}^{\vee}, \alpha_{m}\right\rangle \\
& =-\left\langle\mathbf{y}, \lambda_{m}\right\rangle+\sum_{\alpha \in \Delta_{+} \backslash \Psi} z_{\alpha}\left\langle\alpha^{\vee}, \lambda_{m}\right\rangle,
\end{aligned}
$$

where in the last line we have used Lemma 5.4 and the fact that for $\alpha=\sigma_{m} \alpha_{k} \in \Delta_{2}$ we have

$$
\left\langle\alpha^{\vee}, \lambda_{m}-\alpha_{m}\right\rangle=\left\langle\sigma_{m} \alpha_{k}^{\vee}, \sigma_{m} \lambda_{m}\right\rangle=\left\langle\alpha_{k}^{\vee}, \lambda_{m}\right\rangle=0 .
$$

Since all the factors of the integrand of the right-hand side of (5.18) are periodic functions with its period 1 , we integrate the interval $[0,1]$ with respect to $z_{\alpha}$. Therefore using (5.19), 
(5.23), (5.25) and (5.27) we have

$(5.29) \quad\left(\sigma_{m} F\right)(\mathbf{t}, \mathbf{y} ; \Delta)$

$$
\begin{aligned}
=( & \left.\prod_{\alpha \in \Delta_{+}} \frac{t_{\alpha}}{e^{t_{\alpha}}-1}\right) \int_{0}^{1} \ldots \int_{0}^{1}\left(\prod_{\alpha \in \Delta_{1}} \exp \left(t_{\alpha}\left\{z_{\alpha}\right\}\right)\right) \\
& \times\left(\prod_{\alpha_{i} \in \Psi_{1}} \exp \left(t_{\alpha_{i}}\left\{\left\langle\mathbf{y}, \lambda_{i}\right\rangle-\sum_{\alpha \in \Delta_{+} \backslash \Psi} z_{\alpha}\left\langle\alpha^{\vee}, \lambda_{i}\right\rangle\right\}\right)\right) \\
& \times \exp \left(t_{\alpha_{m}}\left(1-\left\{-\left\langle\mathbf{y}, \lambda_{m}\right\rangle+\sum_{\alpha \in \Delta_{+} \backslash \Psi} z_{\alpha}\left\langle\alpha^{\vee}, \lambda_{m}\right\rangle\right\}\right)\right) \\
& \times\left(\prod_{\alpha_{j} \in \Psi_{2}} \exp \left(t_{\alpha_{j}}\left\{\left\langle\mathbf{y}, \lambda_{j}\right\rangle-\sum_{\alpha \in \Delta_{+} \backslash \Psi} z_{\alpha}\left\langle\alpha^{\vee}, \lambda_{j}\right\rangle\right\}\right)\right) \\
& \times\left(\prod_{\alpha_{i} \in \Psi_{1}} \exp \left(t_{\sigma_{m} \alpha_{i}}\left\{z_{\sigma_{m} \alpha_{i}}\right\}\right)\right) \prod_{\alpha \in \Delta_{+} \backslash \Psi} d z_{\alpha} \\
=( & \left.\prod_{\alpha \in \Delta_{+}} \frac{t_{\alpha}}{e^{t_{\alpha}-1}}\right) \int_{0}^{1} \ldots \int_{0}^{1}\left(\prod_{\alpha \in \Delta_{+} \backslash \Psi} \exp \left(t_{\alpha} z_{\alpha}\right)\right) \\
& \times\left(\prod_{\alpha_{i} \in \Psi \backslash\left\{\alpha_{m}\right\}} \exp \left(t_{\alpha_{i}}\left\{\left\langle\mathbf{y}, \lambda_{i}\right\rangle-\sum_{\alpha \in \Delta_{+} \backslash \Psi} z_{\alpha}\left\langle\alpha^{\vee}, \lambda_{i}\right\rangle\right\}\right)\right) \\
& \times \exp \left(t_{\alpha_{m}}\left(1-\left\{-\left\langle\mathbf{y}, \lambda_{m}\right\rangle+\sum_{\alpha \in \Delta_{+} \backslash \Psi} z_{\alpha}\left\langle\alpha^{\vee}, \lambda_{m}\right\rangle\right\}\right)\right) \prod_{\alpha \in \Delta_{+} \backslash \Psi} d z_{\alpha} \\
=F & (\mathbf{t}, \mathbf{y} ; \Delta),
\end{aligned}
$$

where in the last line, we have used the fact that for any $\alpha_{m} \in \Psi$, there exists a root $\alpha \in \Delta_{+} \backslash \Psi$ such that $\left\langle\alpha^{\vee}, \lambda_{m}\right\rangle \neq 0$ and thus $1-\{-x\}=\{x\}$ for $x \in \mathbb{R} \backslash \mathbb{Z}$ implies that the integrand coincides with that of $F(\mathbf{t}, \mathbf{y} ; \Delta)$ almost everywhere.

Lastly we check the invariance with respect to $\omega \in \Omega$. Since $\omega \in \Omega$ permutes $\Psi$ and leaves $\Delta_{+}$and hence $\Delta_{+} \backslash \Psi$ invariant, we have

$$
\begin{aligned}
(\omega F)(\mathbf{t}, \mathbf{y} ; \Delta) & \left(\prod_{\alpha \in \Delta_{+}} \frac{t_{\omega \alpha}}{e^{t_{\omega \alpha}}-1}\right) \int_{0}^{1} \cdots \int_{0}^{1}\left(\prod_{\alpha \in \Delta_{+} \backslash \Psi} \exp \left(t_{\omega \alpha} x_{\omega \alpha}\right)\right) \\
& \times\left(\prod_{i=1}^{r} \exp \left(t_{\omega \alpha_{i}}\left\{\left\langle\omega^{-1} \mathbf{y}, \lambda_{i}\right\rangle-\sum_{\alpha \in \Delta_{+} \backslash \Psi} x_{\omega \alpha}\left\langle\omega \alpha^{\vee}, \omega \lambda_{i}\right\rangle\right\}\right)\right) \prod_{\alpha \in \Delta_{+} \backslash \Psi} d x_{\omega \alpha} \\
= & \left(\prod_{\alpha \in \Delta_{+}} \frac{t_{\alpha}}{e^{t_{\alpha}}-1}\right) \int_{0}^{1} \cdots \int_{0}^{1}\left(\prod_{\alpha \in \Delta_{+} \backslash \Psi} \exp \left(t_{\alpha} x_{\alpha}\right)\right) \\
& \times\left(\prod_{i=1}^{r} \exp \left(t_{\omega \alpha_{i}}\left\{\left\langle\mathbf{y}, \omega \lambda_{i}\right\rangle-\sum_{\alpha \in \Delta_{+} \backslash \Psi} x_{\alpha}\left\langle\alpha^{\vee}, \omega \lambda_{i}\right\rangle\right\}\right)\right) \prod_{\alpha \in \Delta_{+} \backslash \Psi} d x_{\alpha} \\
= & F(\mathbf{t}, \mathbf{y} ; \Delta) .
\end{aligned}
$$


It is possible to extend the action of $\operatorname{Aut}(\Delta)$ to that of $\widehat{W}$ as follows: For $q \in Q^{\vee}$,

$$
\begin{aligned}
(\tau(q) \mathbf{s})_{\alpha} & =s_{\alpha}, \\
(\tau(q) \mathbf{t})_{\alpha} & =t_{\alpha}, \\
\tau(q) \mathbf{y} & =\mathbf{y}+q .
\end{aligned}
$$

We can observe the periodicity of $S, F$ and $P$ with respect to $\mathbf{y}$ from (4.6), (4.18) and the first line of (4.22). From this periodicity, we have

Theorem 5.8. The action of $\operatorname{Aut}(\Delta)$ is extended to that of $\widehat{W}$ and is given by

$$
\begin{aligned}
& (\tau(q) S)(\mathbf{s}, \mathbf{y} ; \Delta)=S(\mathbf{s}, \mathbf{y} ; \Delta), \\
& (\tau(q) F)(\mathbf{t}, \mathbf{y} ; \Delta)=F(\mathbf{t}, \mathbf{y} ; \Delta), \\
& (\tau(q) P)(\mathbf{k}, \mathbf{y} ; \Delta)=P(\mathbf{k}, \mathbf{y} ; \Delta),
\end{aligned}
$$

for $q \in Q^{\vee}$.

Remark 5.9. Some statements related with $S(\mathbf{s}, \mathbf{y} ; \Delta)$ or $\zeta_{r}(\mathbf{s}, \mathbf{y} ; \Delta)$ in Sections 4 and 5 hold on any regions in $\mathbf{s}$ to which these functions are analytically continued. In particular, in the case $\mathbf{y}=0$, as we noticed at the beginning of Section 4 , the function $\zeta_{r}(\mathbf{s} ; \Delta)=\zeta_{r}(\mathbf{s}, 0 ; \Delta)$ coincides with the zeta-function defined in [12], and its analytic continuation is given in [12, Theorem 6.1].

\section{Generalization of Bernoulli polynomials}

In the previous sections, we have investigated $P(\mathbf{k}, \mathbf{y} ; \Delta)$ as a continuous function in $\mathbf{y}$. In fact, this function is not real analytic in $\mathbf{y}$ in general. However they are piecewise real analytic, and each piece is actually a polynomial in $\mathbf{y}$. In this section we will prove this fact, and will discuss basic properties of those polynomials.

Let $\mathfrak{D}=\left\{\mathbf{y} \in V \mid 0 \leq\left\langle\mathbf{y}, \lambda_{i}\right\rangle \leq 1, \quad(1 \leq i \leq r)\right\}$ be a period-parallelotope of $F(\mathbf{t}, \cdot ; \Delta)$ with its interior. Let $\mathscr{R}$ be the set of all linearly independent subsets $\mathbf{R}=\left\{\beta_{1}, \ldots, \beta_{r-1}\right\} \subset$ $\Delta, \mathfrak{H}_{\mathbf{R}^{\vee}}=\bigoplus_{i=1}^{r-1} \mathbb{R} \beta_{i}^{\vee}$ the hyperplane passing through $\mathbf{R}^{\vee} \cup\{0\}$ and

$$
\mathfrak{H}_{\mathscr{R}}:=\bigcup_{\substack{\mathbf{R} \in \mathscr{R} \\ q \in Q^{\vee}}}\left(\mathfrak{H}_{\mathbf{R}^{\vee}}+q\right) .
$$

Lemma 6.1. We have

$$
\mathfrak{H}_{\mathscr{R}}=\bigcup_{w \in W} w\left(\bigcup_{j=1}^{r}\left(\mathfrak{H}_{\Psi^{\vee} \backslash\left\{\alpha_{j}^{\vee}\right\}}+\mathbb{Z} \alpha_{j}^{\vee}\right)\right) .
$$

The set $\left\{\mathfrak{H}_{\mathbf{R}^{\vee}}+q \mid \mathbf{R} \in \mathscr{R}, q \in Q^{\vee}\right\}$ is locally finite, i.e., for any $\mathbf{y} \in V$, there exists a neighborhood $U(\mathbf{y})$ such that $U(\mathbf{y})$ intersects finitely many of these hyperplanes.

Proof. Fix $\mathbf{R} \in \mathscr{R}$. Then $\tilde{\Delta}^{\vee}=\Delta^{\vee} \cap \mathfrak{H}_{\mathbf{R}^{\vee}}$ is a coroot system so that $\mathbf{R}^{\vee} \subset \tilde{\Delta}^{\vee}$. Let $\mu$ be a nonzero vector normal to $\mathfrak{H}_{\mathbf{R}^{\vee}}$. Then there exists an element $w \in W$ such that $w^{-1} \mu \in C$. Put $w^{-1} \mu=\sum_{j=1}^{r} c_{j} \lambda_{j}$ with $c_{j} \geq 0$. Then $\alpha^{\vee}=\sum_{j=1}^{r} a_{j} \alpha_{j}^{\vee} \in \Delta^{\vee}$ orthogonal to $w^{-1} \mu$ should satisfy $\sum_{j=1}^{r} a_{j} c_{j}=0$. Since $a_{j}$ are all nonpositive or nonnegative, we have $a_{j}=0$ for $j$ such that $c_{j} \neq 0$. Hence $c_{j}=0$ except for only one $j$, because $w^{-1} \tilde{\Delta}^{\vee} \subset \Delta^{\vee}$ is orthogonal to $w^{-1} \mu$ with codimension 1 . That is, $w^{-1} \mu=c \lambda_{j}$ for some $c>0$. Therefore 
$w\left(\Psi^{\vee} \backslash\left\{\alpha_{j}^{\vee}\right\}\right)$ is a fundamental system of $\tilde{\Delta}^{\vee}$ and $\tilde{H}_{\mathbf{R}^{\vee}}=\mathfrak{H}_{w\left(\Psi^{\vee} \backslash\left\{\alpha_{j}^{\vee}\right\}\right)}=w \tilde{H}_{\Psi^{\vee} \backslash\left\{\alpha_{j}^{\vee}\right\}}$. Moreover $Q^{\vee}=w Q^{\vee}=\bigoplus_{i=1}^{r} \mathbb{Z} w \alpha_{i}^{\vee}$, which implies

$$
\mathfrak{H}_{\mathbf{R}^{\vee}}+Q^{\vee}=w \mathfrak{H}_{\Psi^{\vee} \backslash\left\{\alpha_{j}^{\vee}\right\}}+\mathbb{Z} w \alpha_{j}^{\vee},
$$

since $\bigoplus_{i=1, i \neq j}^{r} \mathbb{Z} \alpha_{i}^{\vee} \subset \mathfrak{H}_{\Psi^{\vee} \backslash\left\{\alpha_{j}^{\vee}\right\}}$. This shows that $\mathfrak{H}_{\mathscr{R}}$ is contained in the right-hand side of (6.2). The opposite inclusion is clear. The local finiteness follows from the expression (6.2) and $|W|<\infty$.

Due to the local finiteness shown in Lemma 6.1 and $\partial \mathfrak{D} \subset \mathfrak{H}_{\mathscr{R}}$, we denote by $\mathfrak{D}^{(\nu)}$ each open connected component of $\mathfrak{D} \backslash \mathfrak{H}_{\mathscr{R}}$ so that

$$
\mathfrak{D} \backslash \mathfrak{H}_{\mathscr{R}}=\coprod_{\nu \in \mathfrak{J}} \mathfrak{D}^{(\nu)},
$$

where $\mathfrak{J}$ is a set of indices. Let $\mathscr{V}$ be the set of all linearly independent subsets $\mathbf{V}=$ $\left\{\beta_{1}, \ldots, \beta_{r}\right\} \subset \Delta_{+}$and $\mathscr{A}=\{0,1\}^{n-r}$, where $n=\left|\Delta_{+}\right|$. Let $\mathscr{W}=\mathscr{V} \times \mathscr{A}$. For subsets $u=\left\{u_{1}, \ldots, u_{k}\right\}, v=\left\{v_{1}, \ldots, v_{k+1}\right\} \subset V$ and $c=\left\{c_{1}, \ldots, c_{k+1}\right\} \subset \mathbb{R}$, let

$$
H(\mathbf{y} ; u, v, c)=\operatorname{det}\left(\begin{array}{ccc}
\left\langle u_{1}, v_{1}\right\rangle & \cdots & \left\langle u_{1}, v_{k+1}\right\rangle \\
\vdots & \ddots & \vdots \\
\left\langle u_{k}, v_{1}\right\rangle & \cdots & \left\langle u_{k}, v_{k+1}\right\rangle \\
\left\langle\mathbf{y}, v_{1}\right\rangle+c_{1} & \cdots & \left\langle\mathbf{y}, v_{k+1}\right\rangle+c_{k+1}
\end{array}\right) .
$$

We give a simple description of the polytopes $\mathcal{P}_{\mathbf{m}, \mathbf{y}}$ defined by (4.23). For $\gamma \in \Delta_{+}$, $a \in\{0,1\}$ and $\mathbf{y} \in V$, we define $\mathbf{u}(\gamma, a) \in \mathbb{R}^{n-r}$ by

$$
\mathbf{u}(\gamma, a)_{\alpha}= \begin{cases}(-1)^{1-a}\left\langle\alpha^{\vee}, \lambda_{i}\right\rangle, & \text { if } \gamma=\alpha_{i} \in \Psi, \\ (-1)^{a} \delta_{\alpha \gamma}, & \text { if } \gamma \notin \Psi,\end{cases}
$$

where $\alpha$ runs over $\Delta_{+} \backslash \Psi$, and define $v(\gamma, a ; \mathbf{y}) \in \mathbb{R}$ by

$$
v(\gamma, a ; \mathbf{y})= \begin{cases}(-1)^{1-a}\left(\left\{\left\langle\mathbf{y}, \lambda_{i}\right\rangle\right\}+m_{i}-a\right) & \text { if } \gamma=\alpha_{i} \in \Psi, \\ (-1)^{a} a=-a, & \text { if } \gamma \notin \Psi .\end{cases}
$$

Further we define

$$
\mathcal{H}_{\gamma, a}(\mathbf{y})=\left\{\mathbf{x}=\left(x_{\alpha}\right)_{\alpha \in \Delta_{+} \backslash \Psi} \in \mathbb{R}^{n-r} \mid \mathbf{u}(\gamma, a) \cdot \mathbf{x}=v(\gamma, a ; \mathbf{y})\right\}
$$

and

$$
\mathcal{H}_{\gamma, a}^{+}(\mathbf{y})=\left\{\mathbf{x}=\left(x_{\alpha}\right)_{\alpha \in \Delta_{+} \backslash \Psi} \in \mathbb{R}^{n-r} \mid \mathbf{u}(\gamma, a) \cdot \mathbf{x} \geq v(\gamma, a ; \mathbf{y})\right\},
$$

where for $\mathbf{w}=\left(w_{\alpha}\right), \mathbf{x}=\left(x_{\alpha}\right) \in \mathbb{C}^{n-r}$, we have set

$$
\mathbf{w} \cdot \mathbf{x}=\sum_{\alpha \in \Delta_{+} \backslash \Psi} w_{\alpha} x_{\alpha} .
$$

Then we have

$$
\mathcal{P}_{\mathbf{m}, \mathbf{y}}=\bigcap_{\substack{\gamma \in \Delta_{+} \\ a \in\{0,1\}}} \mathcal{H}_{\gamma, a}^{+}(\mathbf{y}) .
$$

We use the identification $\mathbb{C} \otimes V \simeq \mathbb{C}^{r}$ through $\mathbf{y} \mapsto\left(y_{i}\right)_{i=1}^{r}$ where $y_{i}=\left\langle\mathbf{y}, \lambda_{i}\right\rangle$ with $\langle\cdot, \cdot\rangle$ bilinearly extended over $\mathbb{C}$. For $\mathbf{k}=\left(k_{\alpha}\right)_{\alpha \in \bar{\Delta}} \in \mathbb{N}_{0}^{n}$, we set $|\mathbf{k}|=\sum_{\alpha \in \bar{\Delta}} k_{\alpha}$. 
Theorem 6.2. In each $\mathfrak{D}^{(\nu)}$, the functions $F(\mathbf{t}, \mathbf{y} ; \Delta)$ and $P(\mathbf{k}, \mathbf{y} ; \Delta)$ are real analytic in y. Moreover, $F(\mathbf{t}, \mathbf{y} ; \Delta)$ is analytically continued to a meromorphic function $F^{(\nu)}(\mathbf{t}, \mathbf{y} ; \Delta)$ from each $\mathbb{C}^{n} \times \mathfrak{D}^{(\nu)}$ to the whole space $\mathbb{C}^{n} \times(\mathbb{C} \otimes V)$. Similarly, $P(\mathbf{k}, \mathbf{y} ; \Delta)$ is analytically continued to a polynomial function $B_{\mathbf{k}}^{(\nu)}(\mathbf{y} ; \Delta) \in \mathbb{Q}[\mathbf{y}]$ from each $\mathfrak{D}^{(\nu)}$ to the whole space $\mathbb{C} \otimes V$ with its total degree at most $|\mathbf{k}|+n-r$.

Proof. Throughout this proof, we fix an index $\nu \in \mathfrak{J}$. Note that $\left\{\left\langle\mathbf{y}, \lambda_{i}\right\rangle\right\}=\left\langle\mathbf{y}, \lambda_{i}\right\rangle$ holds for $\mathbf{y} \in \mathfrak{D}$. We show this statement by several steps. In the first three steps, we investigate the dependence of vertices of $\mathcal{P}_{\mathbf{m}, \mathbf{y}}$ on $\mathbf{y} \in \mathfrak{D}^{(\nu)}$ and in the last two steps, by use of this result and triangulation, we show the analyticity of the generating function. We fix $\mathbf{m} \in \mathbb{N}_{0}^{r}$ except in the last step.

(Step 1.) Let $\mathbf{V}=\left\{\beta_{1}, \ldots, \beta_{r}\right\} \subset \Delta_{+}$and $a_{\gamma} \in\{0,1\}$ for $\gamma \in \Delta_{+} \backslash \mathbf{V}$. Consider the intersection of $\left|\Delta_{+} \backslash \mathbf{V}\right|(=n-r)$ hyperplanes

$$
\bigcap_{\gamma \in \Delta_{+} \backslash \mathbf{V}} \mathcal{H}_{\gamma, a_{\gamma}}(\mathbf{y})=\left\{\mathbf{x}=\left(x_{\alpha}\right) \mid \mathbf{u}\left(\gamma, a_{\gamma}\right) \cdot \mathbf{x}=v\left(\gamma, a_{\gamma} ; \mathbf{y}\right) \text { for } \gamma \in \Delta_{+} \backslash \mathbf{V}\right\} .
$$

Then this set consists of the solutions of the system of the $(n-r)$ linear equations

$$
\begin{cases}\sum_{\alpha \in \Delta_{+} \backslash \Psi} x_{\alpha}\left\langle\alpha^{\vee}, \lambda_{j}\right\rangle=\left\langle\mathbf{y}, \lambda_{j}\right\rangle+m_{j}-a_{\alpha_{j}}, & \text { for } \gamma=\alpha_{j} \in \Psi \backslash \mathbf{V}, \\ x_{\gamma}=a_{\gamma}, & \text { for } \gamma \in \Delta_{+} \backslash(\Psi \cup \mathbf{V}) .\end{cases}
$$

Let $I=\left\{i \mid \beta_{i} \in \mathbf{V} \backslash \Psi\right\}$ and $J=\left\{j \mid \alpha_{j} \in \Psi \backslash \mathbf{V}\right\}$. Note that $|I|=|J|=: k$ and $\left\{\beta_{i} \mid i \in I^{c}\right\}=\left\{\alpha_{j} \mid j \in J^{c}\right\}$. The system of the linear equations (6.13) has a unique solution if and only if

$$
\operatorname{det}\left(\left\langle\beta_{i}^{\vee}, \lambda_{j}\right\rangle\right)_{j \in J}^{i \in I} \neq 0
$$

and also if and only if

$$
\mathbf{V} \in \mathscr{V}
$$

since

$$
\begin{aligned}
\left|\operatorname{det}\left(\left\langle\beta_{i}^{\vee}, \lambda_{j}\right\rangle\right)_{1 \leq j \leq r}^{1 \leq i \leq r}\right| & =\left|\operatorname{det}\left(\begin{array}{cc}
\left(\left\langle\beta_{i}^{\vee}, \lambda_{j}\right\rangle\right)_{j \in J}^{i \in I} & \left(\left\langle\beta_{i}^{\vee}, \lambda_{j}\right\rangle\right)_{j \in J^{c}}^{i \in I} \\
\left(\left\langle\alpha_{i}^{\vee}, \lambda_{j}\right\rangle\right)_{j \in J}^{i \in J^{c}} & \left(\left\langle\alpha_{i}^{\vee}, \lambda_{j}\right\rangle\right)_{j \in J^{c}}^{i \in J^{c}}
\end{array}\right)\right| \\
& =\left|\operatorname{det}\left(\begin{array}{cc}
\left(\left\langle\beta_{i}^{\vee}, \lambda_{j}\right\rangle\right)_{j \in J}^{i \in I} & * \\
0 & I_{\left|J^{c}\right|}
\end{array}\right)\right| \\
& =\left|\operatorname{det}\left(\left\langle\beta_{i}^{\vee}, \lambda_{j}\right\rangle\right)_{j \in J}^{i \in I}\right|,
\end{aligned}
$$

where $I_{p}$ is the $p \times p$ identity matrix. We assume (6.15) and denote by $\mathbf{p}(\mathbf{y} ; \mathbf{W})$ the unique solution, where $\mathbf{W}=(\mathbf{V}, \mathbf{A}) \in \mathscr{W}$ with the sequence $\mathbf{A}=\left(a_{\gamma}\right)_{\gamma \in \Delta_{+} \backslash \mathbf{V}}$ regarded as an element of $\mathscr{A}$. We see that $\mathbf{p}(\mathbf{y} ; \mathbf{W})$ depends on $\mathbf{y}$ affine-linearly.

(Step 2.) We define $\xi_{\mathbf{y}}: \mathscr{W} \rightarrow \mathbb{R}^{n-r}$ by

$$
\xi_{\mathbf{y}}: \mathbf{W} \mapsto \mathbf{p}(\mathbf{y} ; \mathbf{W}) .
$$

Any vertex (that is, 0 -face) of $\mathcal{P}_{\mathbf{m}, \mathbf{y}}$ is defined by the intersection of $(n-r)$ hyperplanes by Proposition 2.8, Hence $\operatorname{Vert}\left(\mathcal{P}_{\mathbf{m}, \mathbf{y}}\right) \subset \xi_{\mathbf{y}}(\mathscr{W})$. On the other hand, $\mathcal{P}_{\mathbf{m}, \mathbf{y}}$ is defined by $n$ pairs of inequalities in (4.23). The point $\mathbf{p}(\mathbf{y} ; \mathbf{W})$ is a vertex of $\mathcal{P}_{\mathbf{m}, \mathbf{y}}$ if all of those inequalities hold. We see that $(n-r)$ pairs among them are satisfied, because

$$
\mathbf{p}(\mathbf{y} ; \mathbf{W}) \in \bigcap_{\gamma \in \Delta_{+} \backslash \mathbf{V}} \mathcal{H}_{\gamma, a_{\gamma}}(\mathbf{y})
$$


and also it is easy to check

$$
\mathbf{p}(\mathbf{y} ; \mathbf{W}) \in \bigcap_{\gamma \in \Delta_{+} \backslash \mathbf{V}}\left(\mathcal{H}_{\gamma, 1-a_{\gamma}}^{+}(\mathbf{y}) \backslash \mathcal{H}_{\gamma, 1-a_{\gamma}}(\mathbf{y})\right) .
$$

Therefore $\mathbf{p}(\mathbf{y} ; \mathbf{W}) \in \operatorname{Vert}\left(\mathcal{P}_{\mathbf{m}, \mathbf{y}}\right)$ if and only if the remaining $r$ pairs of inequalities are satisfied, that is,

$$
\mathbf{p}(\mathbf{y} ; \mathbf{W}) \in \bigcap_{\substack{\beta \in \mathbf{V} \\ a \in\{0,1\}}} \mathcal{H}_{\beta, a}^{+}(\mathbf{y})=\left\{\mathbf{x}=\left(x_{\alpha}\right) \mid \mathbf{u}(\beta, a) \cdot \mathbf{x} \geq v(\beta, a ; \mathbf{y}) \text { for } \beta \in \mathbf{V}, a \in\{0,1\}\right\},
$$

or equivalently $\mathbf{x}=\mathbf{p}(\mathbf{y} ; \mathbf{W})$ satisfies $r$ pairs of the linear inequalities

$$
\begin{cases}\left\langle\mathbf{y}, \lambda_{l}\right\rangle+m_{l}-1 \leq \sum_{\alpha \in \Delta_{+} \backslash \Psi} x_{\alpha}\left\langle\alpha^{\vee}, \lambda_{l}\right\rangle \leq\left\langle\mathbf{y}, \lambda_{l}\right\rangle+m_{l}, & \text { for } \beta=\alpha_{l} \in \mathbf{V} \cap \Psi, \\ 0 \leq x_{\beta} \leq 1, & \text { for } \beta \in \mathbf{V} \backslash \Psi .\end{cases}
$$

We see that it depends on $\mathbf{y}$ whether $\mathbf{p}(\mathbf{y} ; \mathbf{W})$ is a vertex, or in other words, whether the solution of (6.13) satisfies (6.21). We will show in the next step that $\mathbf{p}(\mathbf{y} ; \mathbf{W}) \in \mathcal{H}(\mathbf{y} ; \mathbf{W})$ implies $\mathbf{y} \in \mathfrak{H}_{\mathscr{R}}$, where

$$
\mathcal{H}(\mathbf{y} ; \mathbf{W})=\bigcup_{\substack{\beta \in \mathbf{V} \\ a \in\{0,1\}}} \mathcal{H}_{\beta, a}(\mathbf{y}) .
$$

Then for $\mathbf{y} \in \mathfrak{D} \backslash \mathfrak{H}_{\mathscr{R}}$, we can uniquely determine the $(n-r)$ hyperplanes on which the point $\mathbf{p}(\mathbf{y} ; \mathbf{W})$ lies; they are $\left\{\mathcal{H}_{\gamma, a_{\gamma}}(\mathbf{y})\right\}_{\gamma \in \Delta_{+} \backslash \mathbf{V}}$. Therefore $\xi_{\mathbf{y}}$ is an injection.

For $\beta \in \mathbf{V}$ and $a \in\{0,1\}$, we define $f_{\beta, a}: \mathfrak{D} \rightarrow \mathbb{R}$ by

$$
f_{\beta, a}: \mathbf{y} \mapsto \mathbf{u}(\beta, a) \cdot \mathbf{p}(\mathbf{y} ; \mathbf{W})-v(\beta, a ; \mathbf{y}) .
$$

Then for $\mathbf{y} \in \mathfrak{D} \backslash \mathfrak{H}_{\mathscr{R}}$, we have $f_{\beta, a}(\mathbf{y}) \neq 0$ and hence we define

$$
f=\left(f_{\beta, a}\right)_{\beta \in \mathbf{V}, a \in\{0,1\}}: \mathfrak{D} \backslash \mathfrak{H}_{\mathscr{R}} \rightarrow(\mathbb{R} \backslash\{0\})^{2 r} .
$$

Therefore for $\mathbf{y} \in \mathfrak{D} \backslash \mathfrak{H}_{\mathscr{R}}$, the point $\mathbf{p}(\mathbf{y}$; W $)$ is a vertex if and only if $f(\mathbf{y})$ is an element of the connected component $(0, \infty)^{2 r}$. Since each $f_{\beta, a}$ is continuous and hence $f\left(\mathfrak{D}^{(\nu)}\right)$ is connected, we see that for a fixed $\mathbf{W} \in \mathscr{W}$, the point $\mathbf{p}(\mathbf{y} ; \mathbf{W})$ is always a vertex, or never a vertex, on $\mathfrak{D}^{(\nu)}$. Thus

$$
\mathscr{W}_{\mathbf{m}}:=\xi_{\mathbf{y}}^{-1}\left(\operatorname{Vert}\left(\mathcal{P}_{\mathbf{m}, \mathbf{y}}\right)\right) \subset \mathscr{W} \quad\left(\mathbf{y} \in \mathfrak{D}^{(\nu)}\right)
$$

has one-to-one correspondence with $\operatorname{Vert}\left(\mathcal{P}_{\mathbf{m}, \mathbf{y}}\right)$ and is independent of $\mathbf{y}$ on $\mathfrak{D}^{(\nu)}$.

(Step 3.) Now we prove the claim announced just before (6.22). First we show that the condition

$$
\mathbf{p}(\mathbf{y} ; \mathbf{W}) \in \mathcal{H}_{\beta, a_{\beta}}(\mathbf{y})
$$

for some $\beta=\alpha_{l} \in \mathbf{V} \cap \Psi$ and $a_{\beta} \in\{0,1\}$ implies $\mathbf{y} \in \mathfrak{H}_{\mathscr{R}}$. For $\mathbf{x}=\mathbf{p}(\mathbf{y} ; \mathbf{W})$, condition (6.26) is equivalent to

$$
\sum_{\alpha \in \Delta_{+} \backslash \Psi} x_{\alpha}\left\langle\alpha^{\vee}, \lambda_{l}\right\rangle=\left\langle\mathbf{y}, \lambda_{l}\right\rangle+m_{l}-a_{\alpha_{l}} .
$$


From (6.13) and (6.27), we have an overdetermined system with the $|\mathbf{V} \backslash \Psi|=k$ variables $x_{\beta}$ for $\beta \in \mathbf{V} \backslash \Psi$ and the $\left|(\Psi \backslash \mathbf{V}) \cup\left\{\alpha_{l}\right\}\right|=(k+1)$ equations

$$
\sum_{\beta \in \mathbf{V} \backslash \Psi} x_{\beta}\left\langle\beta^{\vee}, \lambda_{j}\right\rangle=\left\langle\mathbf{y}, \lambda_{j}\right\rangle+c_{j},
$$

for $j \in J \cup\{l\}$, where

$$
c_{j}=m_{j}-a_{\alpha_{j}}-\sum_{\gamma \in \Delta_{+} \backslash(\Psi \cup \mathbf{V})} a_{\gamma}\left\langle\gamma^{\vee}, \lambda_{j}\right\rangle \in \mathbb{Z} .
$$

Hence we have

$$
\left(\begin{array}{llll}
x_{\beta_{i_{1}}} & \cdots & x_{\beta_{i_{k}}} & -1
\end{array}\right)\left(\begin{array}{cccc}
\left\langle\beta_{i_{1}}^{\vee}, \lambda_{j_{1}}\right\rangle & \cdots & \left\langle\beta_{i_{1}}^{\vee}, \lambda_{j_{k}}\right\rangle & \left\langle\beta_{i_{1}}^{\vee}, \lambda_{l}\right\rangle \\
\vdots & \ddots & \vdots & \vdots \\
\left\langle\beta_{i_{k}}^{\vee}, \lambda_{j_{1}}\right\rangle & \cdots & \left\langle\beta_{i_{k}}^{\vee}, \lambda_{j_{k}}\right\rangle & \left\langle\beta_{i_{k}}^{\vee}, \lambda_{l}\right\rangle \\
\left\langle\mathbf{y}, \lambda_{j_{1}}\right\rangle+c_{j_{1}} & \cdots & \left\langle\mathbf{y}, \lambda_{j_{k}}\right\rangle+c_{j_{k}} & \left\langle\mathbf{y}, \lambda_{l}\right\rangle+c_{l}
\end{array}\right)=\left(\begin{array}{lll}
0 & \cdots & 0
\end{array}\right),
$$

where we have put $I=\left\{i_{1}, \ldots, i_{k}\right\}$ and $J=\left\{j_{1}, \ldots, j_{k}\right\}$. As the consistency for these equations, we get

$$
H\left(\mathbf{y} ;\left\{\beta_{i}^{\vee}\right\}_{i \in I},\left\{\lambda_{j}\right\}_{j \in J \cup\{l\}},\left\{c_{j}\right\}_{j \in J \cup\{l\}}\right)=0 .
$$

By direct substitution, we see that each element of

$$
\left\{\beta^{\vee}-q\right\}_{\beta \in \mathbf{V} \backslash\left\{\alpha_{l}\right\}} \cup\{-q\}, \quad q=\sum_{j \in J \cup\{l\}} c_{j} \alpha_{j}^{\vee}
$$

satisfies (6.31), while $\alpha_{l}^{\vee}-q$ does not. In fact, if $\mathbf{y}=-q$ or $\mathbf{y}=\beta^{\vee}-q\left(\beta \in(\mathbf{V} \cap \Psi) \backslash\left\{\alpha_{l}\right\}\right)$, then the last row of the matrix is $(0, \ldots, 0)$, and if $\mathbf{y}=\beta_{i_{p}}^{\vee}-q\left(\beta_{i_{p}} \in \mathbf{V} \backslash \Psi\right)$, then the last row is equal to the $p$-th row, and hence (6.31) follows, while if $\mathbf{y}=\alpha_{l}^{\vee}-q$, then the last row of the matrix is $(0, \ldots, 0,1)$ and hence

$$
H\left(\mathbf{y} ;\left\{\beta_{i}^{\vee}\right\}_{i \in I},\left\{\lambda_{j}\right\}_{j \in J \cup\{l\}},\left\{c_{j}\right\}_{j \in J \cup\{l\}}\right)=\operatorname{det}\left(\left\langle\beta_{i}^{\vee}, \lambda_{j}\right\rangle\right)_{j \in J}^{i \in I} \neq 0,
$$

because of (6.14). By (6.15), we see that $\mathbf{V} \backslash\left\{\alpha_{l}\right\} \subset \Delta$ is a linearly independent subset and hence $\left(\mathbf{V} \backslash\left\{\alpha_{l}\right\}\right) \in \mathscr{R}$. It follows that (6.31) represents the hyperplane $\mathfrak{H}_{\mathbf{V}} \backslash\left\{\alpha_{l}^{\vee}\right\}-q \subset \mathfrak{H}_{\mathscr{R}}$. Therefore (6.26) implies $\mathbf{y} \in \mathfrak{H}_{\mathscr{R}}$.

Similarly we see that the condition $\mathbf{p}(\mathbf{y} ; \mathbf{W}) \in \mathcal{H}_{\beta, a_{\beta}}(\mathbf{y})$ for some $\beta=\beta_{l} \in \mathbf{V} \backslash \Psi$ and $a_{\beta} \in\{0,1\}$ yields a hyperplane contained in $\mathfrak{H}_{\mathscr{R}}$ defined by

$$
H\left(\mathbf{y} ;\left\{\beta_{i}^{\vee}\right\}_{i \in I \backslash\{l\}},\left\{\lambda_{j}\right\}_{j \in J},\left\{d_{j}\right\}_{j \in J}\right)=0,
$$

which passes through $r$ points in general position

$$
\left\{\beta^{\vee}-q\right\}_{\beta \in \mathbf{V} \backslash\left\{\beta_{l}\right\}} \cup\{-q\},
$$

where

$$
\begin{gathered}
q=\sum_{j \in J} d_{j} \alpha_{j}^{\vee}, \\
d_{j}=m_{j}-a_{\alpha_{j}}-\sum_{\gamma \in \Delta_{+} \backslash\left(\Psi \cup\left(\mathbf{V} \backslash\left\{\beta_{l}\right\}\right)\right)} a_{\gamma}\left\langle\gamma^{\vee}, \lambda_{j}\right\rangle \in \mathbb{Z} .
\end{gathered}
$$

This completes the proof of our claim.

(Step 4.) We have checked that on $\mathfrak{D}^{(\nu)}$, the vertices $\operatorname{Vert}\left(\mathcal{P}_{\mathbf{m}, \mathbf{y}}\right)$ neither increase nor decrease and are indexed by $\mathscr{W}_{\mathbf{m}}$. By numbering $\mathscr{W}_{\mathbf{m}}$ as $\left\{\mathbf{W}_{1}, \mathbf{W}_{2}, \ldots\right\}$, we denote $\mathbf{p}_{i}(\mathbf{y})=$ 
$\mathbf{p}\left(\mathbf{y} ; \mathbf{W}_{i}\right)$. We see that on $\mathfrak{D}^{(\nu)}$, the polytopes $\mathcal{P}_{\mathbf{m}, \mathbf{y}}$ keep $(n-r)$-dimensional or empty because each vertex is determined by unique $(n-r)$ hyperplanes. Assume that $\mathcal{P}_{\mathbf{m}, \mathbf{y}}$ is not empty. Next we will show that the face poset structure of $\mathcal{P}_{\mathbf{m}, \mathbf{y}}$ is independent of $\mathbf{y}$ on $\mathfrak{D}^{(\nu)}$.

Fix $\mathbf{y}_{0} \in \mathfrak{D}^{(\nu)}$. Consider a face $\mathcal{F}\left(\mathbf{y}_{0}\right)$ of $\mathcal{P}_{\mathbf{m}, \mathbf{y}_{0}}$ and let

$$
\operatorname{Vert}\left(\mathcal{P}_{\mathbf{m}, \mathbf{y}_{0}}\right) \cap \mathcal{F}\left(\mathbf{y}_{0}\right)=\left\{\mathbf{p}_{i_{1}}\left(\mathbf{y}_{0}\right), \ldots, \mathbf{p}_{i_{h}}\left(\mathbf{y}_{0}\right)\right\} .
$$

Then by Proposition 2.8, there exists a subset $\mathcal{J}_{0}=\left\{\left(\gamma_{1}, a_{\gamma_{1}}\right),\left(\gamma_{2}, a_{\gamma_{2}}\right), \ldots\right\} \subset \Delta_{+} \times\{0,1\}$ such that $\left|\mathcal{J}_{0}\right|=n-r-\operatorname{dim} \mathcal{F}\left(\mathbf{y}_{0}\right)$ and

$$
\left\{\mathbf{p}_{i_{1}}\left(\mathbf{y}_{0}\right), \ldots, \mathbf{p}_{i_{h}}\left(\mathbf{y}_{0}\right)\right\} \subset \mathcal{F}\left(\mathbf{y}_{0}\right)=\mathcal{P}_{\mathbf{m}, \mathbf{y}_{0}} \cap \bigcap_{\left(\gamma, a_{\gamma}\right) \in \mathcal{J}_{0}} \mathcal{H}_{\gamma, a_{\gamma}}\left(\mathbf{y}_{0}\right) .
$$

By the definition of $\mathbf{p}_{i_{1}}\left(\mathbf{y}_{0}\right), \ldots, \mathbf{p}_{i_{h}}\left(\mathbf{y}_{0}\right)$ (see (6.12) $)$, we find that for $\left(\gamma, a_{\gamma}\right) \in \Delta_{+} \times\{0,1\}$, the condition

$$
\left\{\mathbf{p}_{i_{1}}\left(\mathbf{y}_{0}\right), \ldots, \mathbf{p}_{i_{h}}\left(\mathbf{y}_{0}\right)\right\} \subset \mathcal{H}_{\gamma, a_{\gamma}}\left(\mathbf{y}_{0}\right)
$$

is equivalent to

$$
\gamma \in \bigcap_{j=1}^{h}\left(\Delta_{+} \backslash \mathbf{V}_{i_{j}}\right), \quad a_{\gamma}=\left(\mathbf{A}_{i_{1}}\right)_{\gamma}=\cdots=\left(\mathbf{A}_{i_{h}}\right)_{\gamma},
$$

where $\mathbf{W}_{i_{j}}=\left(\mathbf{V}_{i_{j}}, \mathbf{A}_{i_{j}}\right)=\xi_{\mathbf{y}_{0}}^{-1}\left(\mathbf{p}_{i_{j}}\left(\mathbf{y}_{0}\right)\right)$. Hence each $\left(\gamma, a_{\gamma}\right) \in \mathcal{J}_{0}$ satisfies (6.41). Assume that there exists a pair $\left(\gamma^{\prime}, a_{\gamma^{\prime}}^{\prime}\right) \notin \mathcal{J}_{0}$ satisfying (6.41), Then

$$
\left\{\mathbf{p}_{i_{1}}\left(\mathbf{y}_{0}\right), \ldots, \mathbf{p}_{i_{h}}\left(\mathbf{y}_{0}\right)\right\} \subset \bigcap_{\left(\gamma, a_{\gamma}\right) \in \mathcal{J}_{0}^{\prime}} \mathcal{H}_{\gamma, a_{\gamma}}\left(\mathbf{y}_{0}\right),
$$

where $\mathcal{J}_{0}^{\prime}=\mathcal{J}_{0} \cup\left\{\left(\gamma^{\prime}, a_{\gamma^{\prime}}^{\prime}\right)\right\}$. Hence by (2.19), we have

$$
\mathcal{F}\left(\mathbf{y}_{0}\right)=\operatorname{Conv}\left\{\mathbf{p}_{i_{1}}\left(\mathbf{y}_{0}\right), \ldots, \mathbf{p}_{i_{h}}\left(\mathbf{y}_{0}\right)\right\} \subset \bigcap_{\left(\gamma, a_{\gamma}\right) \in \mathcal{J}_{0}^{\prime}} \mathcal{H}_{\gamma, a_{\gamma}}\left(\mathbf{y}_{0}\right),
$$

and in particular,

$$
\mathbf{p}_{i_{1}}\left(\mathbf{y}_{0}\right) \in \bigcap_{\left(\gamma, a_{\gamma}\right) \in \mathcal{J}_{0}^{\prime}} \mathcal{H}_{\gamma, a_{\gamma}}\left(\mathbf{y}_{0}\right) .
$$

Since $(n-r)$ hyperplanes on which $\mathbf{p}_{i_{1}}\left(\mathbf{y}_{0}\right)$ lies are uniquely determined and their intersection consists of only $\mathbf{p}_{i_{1}}\left(\mathbf{y}_{0}\right)$, their normal vectors $\left\{\mathbf{u}\left(\gamma, a_{\gamma}\right)\right\}_{\left(\gamma, a_{\gamma}\right) \in \mathcal{J}_{0}^{\prime}}$ must be linearly independent. It follows that the dimension of the right-hand side of (6.43) is $n-r-\left|\mathcal{J}_{0}^{\prime}\right|<n-r-\left|\mathcal{J}_{0}\right|=\operatorname{dim} \mathcal{F}\left(\mathbf{y}_{0}\right)$, which contradicts. Hence (6.41) is also a sufficient condition for $\left(\gamma, a_{\gamma}\right) \in \mathcal{J}_{0}$.

By definition, we have

$$
\left\{\mathbf{p}_{i_{1}}(\mathbf{y}), \ldots, \mathbf{p}_{i_{h}}(\mathbf{y})\right\} \subset \bigcap_{\left(\gamma, a_{\gamma}\right) \in \mathcal{J}_{0}} \mathcal{H}_{\gamma, a_{\gamma}}(\mathbf{y}),
$$

for all $\mathbf{y} \in \mathfrak{D}^{(\nu)}$. Define

$$
\mathcal{F}(\mathbf{y})=\mathcal{P}_{\mathbf{m}, \mathbf{y}} \cap \bigcap_{\left(\gamma, a_{\gamma}\right) \in \mathcal{J}_{0}} \mathcal{H}_{\gamma, a_{\gamma}}(\mathbf{y}) .
$$


Then $\left\{\mathbf{p}_{i_{1}}(\mathbf{y}), \ldots, \mathbf{p}_{i_{h}}(\mathbf{y})\right\} \subset \mathcal{F}(\mathbf{y})$ and by Proposition 2.7, we see that $\mathcal{F}(\mathbf{y})$ is a face. Fix another $\mathbf{y}_{1} \in \mathfrak{D}^{(\nu)}$. Then by the argument at the beginning of this step, there exists a subset $\mathcal{J}_{1} \subset \Delta_{+} \times\{0,1\}$ such that $\left|\mathcal{J}_{1}\right|=n-r-\operatorname{dim} \mathcal{F}\left(\mathbf{y}_{1}\right)$ and

$$
\begin{aligned}
\operatorname{Vert}\left(\mathcal{P}_{\mathbf{m}, \mathbf{y}_{1}}\right) \cap \mathcal{F}\left(\mathbf{y}_{1}\right) & =\left\{\mathbf{p}_{i_{1}}\left(\mathbf{y}_{1}\right), \ldots, \mathbf{p}_{i_{h}}\left(\mathbf{y}_{1}\right), \mathbf{p}_{i_{h+1}}\left(\mathbf{y}_{1}\right), \ldots, \mathbf{p}_{i_{h^{\prime}}}\left(\mathbf{y}_{1}\right)\right\} \\
& \subset \mathcal{F}\left(\mathbf{y}_{1}\right)=\mathcal{P}_{\mathbf{m}, \mathbf{y}_{1}} \cap \bigcap_{\left(\gamma, a_{\gamma}\right) \in \mathcal{J}_{1}} \mathcal{H}_{\gamma, a_{\gamma}}\left(\mathbf{y}_{1}\right),
\end{aligned}
$$

with $h^{\prime} \geq h$ (because all of $\mathbf{p}_{i_{1}}\left(\mathbf{y}_{1}\right), \ldots, \mathbf{p}_{i_{h}}\left(\mathbf{y}_{1}\right)$ are vertices of $\mathcal{F}\left(\mathbf{y}_{1}\right)$, while so far we cannot exclude the possibility of the existence of other vertices on $\mathcal{F}\left(\mathbf{y}_{1}\right)$ ). Since each $\left(\gamma, a_{\gamma}\right) \in \mathcal{J}_{1}$ satisfies

$$
\gamma \in \bigcap_{j=1}^{h^{\prime}}\left(\Delta_{+} \backslash \mathbf{V}_{i_{j}}\right), \quad a_{\gamma}=\left(\mathbf{A}_{i_{1}}\right)_{\gamma}=\cdots=\left(\mathbf{A}_{i_{h^{\prime}}}\right)_{\gamma},
$$

which is equal to or stronger than condition (6.41), we have $\mathcal{J}_{1} \subset \mathcal{J}_{0}$. On the other hand, by comparing (6.46) and (6.47), we see that each $\left(\gamma, a_{\gamma}\right) \in \mathcal{J}_{0}$ satisfies (6.48). As shown in the previous paragraph, condition (6.48) is sufficient for $\left(\gamma, a_{\gamma}\right) \in \mathcal{J}_{1}$, which implies $\mathcal{J}_{0}=\mathcal{J}_{1}$ and hence $\operatorname{dim} \mathcal{F}\left(\mathbf{y}_{1}\right)=\operatorname{dim} \mathcal{F}\left(\mathbf{y}_{0}\right)$. If $h^{\prime}>h$, then (6.48) implies

$$
\left\{\mathbf{p}_{i_{1}}\left(\mathbf{y}_{0}\right), \ldots, \mathbf{p}_{i_{h}}\left(\mathbf{y}_{0}\right), \mathbf{p}_{i_{h+1}}\left(\mathbf{y}_{0}\right), \ldots, \mathbf{p}_{i_{h^{\prime}}}\left(\mathbf{y}_{0}\right)\right\} \subset \mathcal{F}\left(\mathbf{y}_{0}\right),
$$

which contradicts to (6.38) and hence $h^{\prime}=h$. Therefore for all $\mathbf{y} \in \mathfrak{D}^{(\nu)}$, we see that all faces of $\mathcal{P}_{\mathbf{m}, \mathbf{y}}$ are determined at $\mathbf{y}_{0}$ and are described in the form (6.46), and we have

$$
\operatorname{Vert}\left(\mathcal{P}_{\mathbf{m}, \mathbf{y}}\right) \cap \mathcal{F}(\mathbf{y})=\left\{\mathbf{p}_{i_{1}}(\mathbf{y}), \ldots, \mathbf{p}_{i_{h}}(\mathbf{y})\right\} .
$$

Assume that $\mathcal{F}^{\prime}\left(\mathbf{y}_{0}\right) \subset \mathcal{F}\left(\mathbf{y}_{0}\right)$ for faces $\mathcal{F}^{\prime}\left(\mathbf{y}_{0}\right), \mathcal{F}\left(\mathbf{y}_{0}\right)$ of $\mathcal{P}_{\mathbf{m}, \mathbf{y}_{0}}$. Then by (2.19), it is equivalent to

$$
\operatorname{Vert}\left(\mathcal{P}_{\mathbf{m}, \mathbf{y}_{0}}\right) \cap \mathcal{F}^{\prime}\left(\mathbf{y}_{0}\right) \subset \operatorname{Vert}\left(\mathcal{P}_{\mathbf{m}, \mathbf{y}_{0}}\right) \cap \mathcal{F}\left(\mathbf{y}_{0}\right) .
$$

By applying $\xi_{\mathbf{y}_{0}}^{-1}$, we obtain an equivalent condition independent of $\mathbf{y}$ and hence $\mathcal{F}^{\prime}\left(\mathbf{y}_{1}\right) \subset$ $\mathcal{F}\left(\mathbf{y}_{1}\right)$. Therefore the face poset structure is indeed independent of $\mathbf{y}$ on $\mathfrak{D}^{(\nu)}$.

(Step 5.) By Theorem 2.4, we have a triangulation of $\mathcal{P}_{\mathbf{m}, \mathbf{y}}$ with $(n-r)$-dimensional simplexes $\sigma_{l, \mathbf{m}, \mathbf{y}}$ as

$$
\mathcal{P}_{\mathbf{m}, \mathbf{y}}=\bigcup_{l=1}^{L(\mathbf{m}, \mathbf{y})} \sigma_{l, \mathbf{m}, \mathbf{y}}
$$

where $L(\mathbf{m}, \mathbf{y})$ is the number of the simplexes. From the previous step and by Remark 2.5. we see that this triangulation does not depend on $\mathbf{y}$ up to the order of simplexes, i.e.,

$$
\{\mathcal{I}(1, \mathbf{m}, \mathbf{y}), \ldots, \mathcal{I}(L(\mathbf{m}, \mathbf{y}), \mathbf{m}, \mathbf{y})\}
$$

is independent of $\mathbf{y}$, where $\mathcal{I}(l, \mathbf{m}, \mathbf{y})$ is the set of all indices of the vertices of $\sigma_{l, \mathbf{m}, \mathbf{y}}$. Reordering $\sigma_{l, \mathbf{m}, \mathbf{y}}$ with respect to $l$ if necessary, we assume that each $\mathcal{I}(l, \mathbf{m})=\mathcal{I}(l, \mathbf{m}, \mathbf{y})$ is independent of $\mathbf{y}$ on $\mathfrak{D}^{(\nu)}$. Note that $|\mathcal{I}(l, \mathbf{m})|=n-r+1$. Let $L(\mathbf{m})=L(\mathbf{m}, \mathbf{y})$ if $\mathcal{P}_{\mathbf{m}, \mathbf{y}}$ is not empty, and $L(\mathbf{m})=0$ otherwise.

By (6.52) and Lemma 2.9, we find that the integral in the third expression of (4.22) is

$$
(n-r) ! \sum_{l=1}^{L(\mathbf{m})} \operatorname{Vol}\left(\sigma_{l, \mathbf{m}, \mathbf{y}}\right) \sum_{i \in \mathcal{I}(l, \mathbf{m})} \frac{e^{\mathbf{t}^{*} \cdot \mathbf{p}_{i}(\mathbf{y})}}{\prod_{\substack{j \in \mathcal{I}(l, \mathbf{m}) \\ j \neq i}} \mathbf{t}^{*} \cdot\left(\mathbf{p}_{i}(\mathbf{y})-\mathbf{p}_{j}(\mathbf{y})\right)},
$$


where $\mathbf{t}^{*}=\left(t_{\alpha}^{*}\right)$ with $t_{\alpha}^{*}=t_{\alpha}-\sum_{i=1}^{r} t_{\alpha_{i}}\left\langle\alpha^{\vee}, \lambda_{i}\right\rangle$. We see that $\operatorname{Vol}\left(\sigma_{l, \mathbf{m}, \mathbf{y}}\right)$ is a polynomial function in $\mathbf{y}=\left(y_{i}\right)_{i=1}^{r}$ with rational coefficients and its total degree at most $n-r$ on $\mathfrak{D}^{(\nu)}$ due to (2.25), because in Step 1 we have shown that $\mathbf{p}_{i}(\mathbf{y})$ depends on $\mathbf{y}$ affine-linearly. Therefore from (4.22), we have the generating function

$$
F(\mathbf{t}, \mathbf{y} ; \Delta)=\left(\prod_{\alpha \in \Delta_{+}} \frac{t_{\alpha}}{e^{t_{\alpha}}-1}\right)^{(n-r+1) \sum_{\mathbf{m}} L(\mathbf{m})} \frac{f_{j}(\mathbf{y}) e^{h_{j}(\mathbf{t}, \mathbf{y})}}{g_{j}(\mathbf{t}, \mathbf{y})}
$$

which is valid for all $\mathbf{y} \in \mathfrak{D}^{(\nu)}$, where $f_{j} \in \mathbb{Q}[\mathbf{y}]$ with its total degree at most $n-r$ and $g_{j} \in \mathbb{Z}[\mathbf{t}, \mathbf{y}], h_{j} \in \mathbb{Q}[\mathbf{t}, \mathbf{y}]$ are of the form

$$
\begin{array}{ll}
g_{j}(\mathbf{t}, \mathbf{y})=\sum_{\alpha \in \Delta_{+}}\left(\left\langle\phi_{\alpha}, \mathbf{y}\right\rangle+c_{\alpha}\right) t_{\alpha}, & \phi_{\alpha} \in P, \quad c_{\alpha} \in \mathbb{Z}, \\
h_{j}(\mathbf{t}, \mathbf{y})=\sum_{\alpha \in \Delta_{+}}\left(\left\langle\varphi_{\alpha}, \mathbf{y}\right\rangle+d_{\alpha}\right) t_{\alpha}, & \varphi_{\alpha} \in \mathbb{Q} \otimes P, \quad d_{\alpha} \in \mathbb{Q} .
\end{array}
$$

We see from (6.55) that $F(\mathbf{t}, \mathbf{y} ; \Delta)$ is meromorphically continued from $\mathbb{C}^{n} \times \mathfrak{D}^{(\nu)}$ to the whole space $\mathbb{C}^{n} \times(\mathbb{C} \otimes V)$, and that $P(\mathbf{k}, \mathbf{y} ; \Delta)$ is analytically continued to a polynomial function in $\mathbf{y}=\left(y_{i}\right)_{i=1}^{r}$ with rational coefficients and its total degree at most $|\mathbf{k}|+n-r$ by (4.20) and Lemma 2.10 .

Theorem 6.3. The function $P(\mathbf{k}, \mathbf{y} ; \Delta)$ is not real analytic in $\mathbf{y}$ on $V$ unless $P(\mathbf{k}, \mathbf{y} ; \Delta)$ is a constant.

Proof. By Theorem 5.8, we see that for $\mathbf{k} \in \mathbb{N}_{0}^{n}, P(\mathbf{k}, \mathbf{y} ; \Delta)$ is a periodic function in $\mathbf{y}$ with its periods $\Psi^{\vee}$, while by Theorem 6.2, $P(\mathbf{k}, \mathbf{y} ; \Delta)$ is a polynomial function in $\mathbf{y}$ on some open region. Therefore such a polynomial expression cannot be extended to the whole space unless $P(\mathbf{k}, \mathbf{y} ; \Delta)$ is a constant. This implies that there are some points on $\mathfrak{H}_{\mathscr{R}}$, at which $P(\mathbf{k}, \mathbf{y} ; \Delta)$ is not real analytic.

The polynomials $B_{\mathbf{k}}^{(\nu)}(\mathbf{y} ; \Delta)$ may be regarded as (root-system theoretic) generalizations of Bernoulli polynomials. For instance, they possess the following property.

Theorem 6.4. Assume that $\Delta$ is an irreducible root system and is not of type $A_{1}$. For $\mathbf{k} \in \mathbb{N}_{0}^{n}, \mathbf{y} \in \partial \mathfrak{D}^{(\nu)}$ and $\mathbf{y}^{\prime} \in \partial \mathfrak{D}^{\left(\nu^{\prime}\right)}$ with $\mathbf{y} \equiv \mathbf{y}^{\prime}\left(\bmod Q^{\vee}\right)$, we have

$$
B_{\mathbf{k}}^{(\nu)}(\mathbf{y} ; \Delta)=B_{\mathbf{k}}^{\left(\nu^{\prime}\right)}\left(\mathbf{y}^{\prime} ; \Delta\right) .
$$

Proof. If $\mathbf{y}=\mathbf{y}^{\prime}$, then the result follows from the continuity proved in Lemma 3.4. If $\mathbf{y} \neq \mathbf{y}^{\prime}$, but $\mathbf{y} \equiv \mathbf{y}^{\prime}\left(\bmod Q^{\vee}\right)$, we also use the periodicity.

This theorem also holds in the $A_{1}$ case with $k \neq 1$ and can be regarded as a multiple analogue of the formula for the classical Bernoulli polynomials

$$
B_{k}(0)=B_{k}(1)
$$

for $k \neq 1$. Moreover the formula

$$
B_{k}(1-y)=(-1)^{k} B_{k}(y)
$$

is well-known. In the rest of this section we will show the results analogous to the above formula for $B_{\mathbf{k}}^{(\nu)}(\mathbf{y} ; \Delta)$ (Theorem [6.6), and its vector-valued version (Theorem 6.8). The latter gives a finite dimensional representation of Weyl groups. In this framework, (6.60) can be interpreted as an action of the Weyl group of type $A_{1}$ (Example 7.1). These results will not be used in the present paper, but we insert this topic because of its own interest. 
Lemma 6.5. Fix $w \in \operatorname{Aut}(\Delta)$ and $\nu \in \mathfrak{J}$. Then there exist unique $q_{w, \nu} \in Q^{\vee}$ and $\kappa \in \mathfrak{J}$ such that

$$
\tau\left(q_{w, \nu}\right) w \mathfrak{D}^{(\nu)}=\mathfrak{D}^{(\kappa)} .
$$

Thus Aut $(\Delta)$ acts on $\mathfrak{J}$ as $w(\nu)=\kappa$. Moreover $q_{v, w(\nu)}+v q_{w, \nu}=q_{v w, \nu}$ for $v, w \in \operatorname{Aut}(\Delta)$.

Proof. It can be easily seen from the definition (6.1) that $\mathfrak{H}_{\mathscr{R}}$ is $\widehat{W}$-invariant. Therefore $\widehat{W}$ acts on $V \backslash \mathfrak{H}_{\mathscr{R}}$ as homeomorphisms and a connected component is mapped to another one.

Fix $\mathbf{y} \in w \mathfrak{D}^{(\nu)}$. There exists a unique $q \in Q^{\vee}$ such that $0 \leq\left\langle\tau(q) \mathbf{y}, \lambda_{j}\right\rangle<1$ for $1 \leq$ $j \leq r$, that is, $q=-\sum_{j=1}^{r} a_{j} \alpha_{j}^{\vee} \in Q^{\vee}$, where $a_{j}=\left[\left\langle\mathbf{y}, \lambda_{j}\right\rangle\right]$ is the integer part of $\left\langle\mathbf{y}, \lambda_{j}\right\rangle$. Denote this $q$ by $q_{w, \nu}$. Then $\tau\left(q_{w, \nu}\right) \mathbf{y} \in \mathfrak{D}^{(\kappa)}$ for some $\kappa \in \mathfrak{J}$ and thus $\tau\left(q_{w, \nu}\right) w \mathfrak{D}^{(\nu)}=\mathfrak{D}^{(\kappa)}$.

Let $w, w^{\prime} \in \operatorname{Aut}(\Delta)$. Assume

$$
\tau(q) w \mathfrak{D}^{(\nu)}=\mathfrak{D}^{(\kappa)}, \quad \tau\left(q^{\prime}\right) w^{\prime} \mathfrak{D}^{(\kappa)}=\mathfrak{D}^{\left(\kappa^{\prime}\right)}, \quad \tau\left(q^{\prime \prime}\right) w^{\prime} w \mathfrak{D}^{(\nu)}=\mathfrak{D}^{\left(\kappa^{\prime \prime}\right)},
$$

for $q, q^{\prime}, q^{\prime \prime} \in Q^{\vee}$ and $\nu, \kappa, \kappa^{\prime}, \kappa^{\prime \prime} \in \mathfrak{J}$. Then we have

$$
\begin{aligned}
\mathfrak{D}^{\left(\kappa^{\prime}\right)} & =\tau\left(q^{\prime}\right) w^{\prime} \tau(q) w \mathfrak{D}^{(\nu)} \\
& =\tau\left(q^{\prime}+w^{\prime} q\right) w^{\prime} w \mathfrak{D}^{(\nu)} .
\end{aligned}
$$

By the uniqueness, we have $q^{\prime}+w^{\prime} q=q^{\prime \prime}$ and $\kappa^{\prime}=\kappa^{\prime \prime}$.

Theorem 6.6. For $w \in \operatorname{Aut}(\Delta)$,

$$
B_{\mathbf{k}}^{(\nu)}\left(\tau\left(q_{w^{-1}, w(\nu)}\right) w^{-1} \mathbf{y} ; \Delta\right)=\left(\prod_{\alpha \in \Delta_{w}}(-1)^{-k_{\alpha}}\right) B_{w \mathbf{k}}^{(w(\nu))}(\mathbf{y} ; \Delta) .
$$

Proof. By Theorems 5.5] and 5.8, we have

$$
\begin{aligned}
P\left(\mathbf{k}, \tau\left(q_{w^{-1}, w(\nu)}\right) w^{-1} \mathbf{y} ; \Delta\right) & =P\left(\mathbf{k}, w^{-1} \mathbf{y} ; \Delta\right) \\
& =\left(\prod_{\alpha \in \Delta_{w^{-1}}}(-1)^{-k_{w^{-1}}}\right) P(w \mathbf{k}, \mathbf{y} ; \Delta) \\
& =\left(\prod_{\alpha \in \Delta_{w}}(-1)^{-k_{\alpha}}\right) P(w \mathbf{k}, \mathbf{y} ; \Delta),
\end{aligned}
$$

where we have used $w^{-1} \Delta_{w^{-1}}=-\Delta_{w}$. By (6.61) in Lemma 6.5 with replacing $w, \nu$ by $w^{-1}, w(\nu)$ respectively, we have

$$
\tau\left(q_{w^{-1}, w(\nu)}\right) w^{-1} \mathfrak{D}^{(w(\nu))}=\mathfrak{D}^{(\nu)} .
$$

Hence $\mathbf{y} \in \mathfrak{D}^{(w(\nu))}$ implies $\tau\left(q_{w^{-1}, w(\nu)}\right) w^{-1} \mathbf{y} \in \mathfrak{D}^{(\nu)}$. Therefore we obtain

$$
\begin{aligned}
P\left(\mathbf{k}, \tau\left(q_{w^{-1}, w(\nu)}\right) w^{-1} \mathbf{y} ; \Delta\right) & =B_{\mathbf{k}}^{(\nu)}\left(\tau\left(q_{w^{-1}, w(\nu)}\right) w^{-1} \mathbf{y} ; \Delta\right), \\
P(\mathbf{k}, \mathbf{y} ; \Delta) & =B_{\mathbf{k}}^{(w(\nu))}(\mathbf{y} ; \Delta),
\end{aligned}
$$

by Theorem 6.2. The theorem of identity implies (6.64)

Let $\mathfrak{P}$ be the $\mathbb{Q}$-vector space of all vector-valued polynomial functions of the form $f=\left(f_{\nu}\right)_{\nu \in \mathfrak{J}}: V \rightarrow \mathbb{R}^{|\mathfrak{J}|}$ with $f_{\nu} \in \mathbb{Q}[\mathbf{y}]$. We define a linear map $\phi(w): \mathfrak{P} \rightarrow \mathfrak{P}$ for $w \in \operatorname{Aut}(\Delta)$ by

$$
(\phi(w) f)_{\nu}(\mathbf{y})=f_{w^{-1}(\nu)}\left(\tau\left(q_{w^{-1}, \nu}\right) w^{-1} \mathbf{y}\right) .
$$

Theorem 6.7. The pair $(\phi, \mathfrak{P})$ is a representation of $\operatorname{Aut}(\Delta)$. 
Proof. For $v, w \in \operatorname{Aut}(\Delta)$, we have

$$
\begin{aligned}
(\phi(v) \phi(w) f)_{\nu}(\mathbf{y}) & =(\phi(w) f)_{v^{-1}(\nu)}\left(\tau\left(q_{v^{-1}, \nu}\right) v^{-1} \mathbf{y}\right) \\
& =f_{w^{-1} v^{-1}(\nu)}\left(\tau\left(q_{w^{-1}, v^{-1}(\nu)}\right) w^{-1} \tau\left(q_{v^{-1}, \nu}\right) v^{-1} \mathbf{y}\right) \\
& =f_{(v w)^{-1}(\nu)}\left(\tau\left(q_{w^{-1}, v^{-1}(\nu)}+w^{-1} q_{v^{-1}, \nu}\right)(v w)^{-1} \mathbf{y}\right) .
\end{aligned}
$$

Since by Lemma 6.5 we have $q_{w^{-1}, v^{-1}(\nu)}+w^{-1} q_{v^{-1}, \nu}=q_{(v w)^{-1}, \nu}$, we obtain $\phi(v w)=$ $\phi(v) \phi(w)$.

Define $\mathbf{B}_{\mathbf{k}}^{(\nu)} \in \mathfrak{P}$ for $\mathbf{k} \in \mathbb{N}_{0}^{n}$ and $\nu \in \mathfrak{J}$ by

$$
\left(\mathbf{B}_{\mathbf{k}}^{(\nu)}\right)_{\kappa}(\mathbf{y})=\left(\mathbf{B}_{\mathbf{k}}^{(\nu)}\right)_{\kappa}(\mathbf{y} ; \Delta)= \begin{cases}B_{\mathbf{k}}^{(\nu)}(\mathbf{y} ; \Delta), & \text { if } \nu=\kappa, \\ 0, & \text { otherwise }\end{cases}
$$

and let

$$
\mathfrak{B} \overline{(\mathbf{k}, \nu)}=\sum_{\left(\mathbf{k}^{\prime}, \nu^{\prime}\right) \in \overline{(\mathbf{k}, \nu)}} \mathbb{Q} \mathbf{B}_{\mathbf{k}^{\prime}}^{\left(\nu^{\prime}\right)} \subset \mathfrak{P},
$$

where $\overline{(\mathbf{k}, \nu)}$ is an element of the orbit space $\left(\mathbb{N}_{0}^{n} \times \mathfrak{J}\right) / \operatorname{Aut}(\Delta)$.

Theorem 6.8. The vector subspace $\mathfrak{B} \overline{(\mathbf{k}, \nu)}$ is a finite dimensional $\operatorname{Aut}(\Delta)$-invariant subspace and the action is

$$
\phi(w) \mathbf{B}_{\mathbf{k}}^{(\nu)}=\left(\prod_{\alpha \in \Delta_{w}}(-1)^{-k_{\alpha}}\right) \mathbf{B}_{w \mathbf{k}}^{(w))} .
$$

Proof. If $\kappa=w(\nu)$, then we have

$$
\begin{aligned}
\left(\phi(w) \mathbf{B}_{\mathbf{k}}^{(\nu)}\right)_{\kappa}(\mathbf{y} ; \Delta) & =B_{\mathbf{k}}^{(\nu)}\left(\tau\left(q_{w^{-1}, w(\nu)}\right) w^{-1} \mathbf{y} ; \Delta\right) \\
& =\left(\prod_{\alpha \in \Delta_{w}}(-1)^{-k_{\alpha}}\right) B_{w \mathbf{k}}^{(w(\nu))}(\mathbf{y} ; \Delta),
\end{aligned}
$$

by Theorem 6.6 and otherwise

$$
\left(\phi(w) \mathbf{B}_{\mathbf{k}}^{(\nu)}\right)_{\kappa}(\mathbf{y})=0
$$

Thus we obtain (6.73).

\section{EXAMPLES}

Example 7.1. The set of positive roots of type $A_{1}$ consists of only one root $\alpha_{1}$. Hence we have $\Delta_{+}=\Psi=\left\{\alpha_{1}\right\}$ and $2 \rho^{\vee}=\alpha_{1}^{\vee}$. We set $t=t_{\alpha_{1}}$ and $y=\left\langle\mathbf{y}, \lambda_{1}\right\rangle$. Then by Theorem 4.5. we obtain the generating function $F\left(\mathbf{t}, \mathbf{y} ; A_{1}\right)$ as

$$
F\left(\mathbf{t}, \mathbf{y} ; A_{1}\right)=\frac{t}{e^{t}-1} e^{t\{y\}}
$$

Since $\mathfrak{D}=\{\mathbf{y} \mid 0 \leq y \leq 1\}$ and $\mathscr{R}=\{\emptyset\}$, we have $\mathfrak{H}_{\mathscr{R}}=Q^{\vee}$ and hence $\mathfrak{D} \backslash \mathfrak{H}_{\mathscr{R}}$ consists of only one connected component $\mathfrak{D} \backslash \mathfrak{H}_{\mathscr{R}}=\mathfrak{D}=\mathfrak{D}^{(1)}$. Therefore we have

$$
F^{(1)}\left(\mathbf{t}, \mathbf{y} ; A_{1}\right)=\frac{t e^{t y}}{e^{t}-1}
$$

for $\mathbf{y} \in \mathfrak{D}^{(1)}$, which coincides with the generating function of the classical Bernoulli polynomials. Since $\mathfrak{J}=\{1\}$, we see that $\mathbf{B}_{\mathbf{k}}^{(1)}\left(\mathbf{y} ; A_{1}\right)$ consists of only one component, that is, the classical Bernoulli polynomial $B_{k}(y)$. The extended Weyl group $\operatorname{Aut}(\Delta)$ is $\left\{\operatorname{id}, \sigma_{1}\right\}$. For 
$\mathbf{y} \in \mathfrak{D}^{(1)}$, we have $\sigma_{1} \mathbf{y}=-\mathbf{y}$ and hence $\tau\left(\alpha_{1}^{\vee}\right) \sigma_{1} \mathbf{y}=\alpha_{1}^{\vee}-\mathbf{y} \in \mathfrak{D}^{(1)}$ due to $0<\left\langle\alpha_{1}^{\vee}-\mathbf{y}, \lambda_{1}\right\rangle<$ 1 , which implies that the action on $\mathbb{Q}[\mathbf{y}]$ is given by $\left(\phi\left(\sigma_{1}\right) f\right)(\mathbf{y})=f\left(\alpha_{1}^{\vee}-\mathbf{y}\right)$. Therefore we have the well-known property

$$
\left(\phi\left(\sigma_{1}\right) \mathbf{B}_{\mathbf{k}}^{(1)}\right)\left(\mathbf{y} ; A_{1}\right)=B_{k}(1-y)=(-1)^{k} B_{k}(y)=(-1)^{k} \mathbf{B}_{\mathbf{k}}^{(1)}\left(\mathbf{y} ; A_{1}\right) .
$$

Example 7.2. In the root system of type $A_{2}$, we have $\Delta_{+}=\left\{\alpha_{1}, \alpha_{2}, \alpha_{1}+\alpha_{2}\right\}$ and $\Psi=\left\{\alpha_{1}, \alpha_{2}\right\}$. Then $\mathscr{R}=\Delta_{+}$and from Lemma 6.1 we have

$$
\mathfrak{H}_{\mathscr{R}}=\left(\mathbb{R} \alpha_{1}^{\vee}+\mathbb{Z} \alpha_{2}^{\vee}\right) \cup\left(\mathbb{R} \alpha_{2}^{\vee}+\mathbb{Z} \alpha_{1}^{\vee}\right) \cup\left(\mathbb{R}\left(\alpha_{1}^{\vee}+\alpha_{2}^{\vee}\right)+\mathbb{Z} \alpha_{1}^{\vee}\right) .
$$

We see that $\mathfrak{D} \backslash \mathfrak{H}_{\mathscr{R}}=\mathfrak{D}^{(1)} \amalg \mathfrak{D}^{(2)}$, where

$$
\begin{aligned}
& \mathfrak{D}^{(1)}=\left\{\mathbf{y} \mid 0<y_{2}<y_{1}<1\right\}, \\
& \mathfrak{D}^{(2)}=\left\{\mathbf{y} \mid 0<y_{1}<y_{2}<1\right\},
\end{aligned}
$$

with $y_{1}=\left\langle\mathbf{y}, \lambda_{1}\right\rangle$ and $y_{2}=\left\langle\mathbf{y}, \lambda_{2}\right\rangle$. Let $t_{1}=t_{\alpha_{1}}, t_{2}=t_{\alpha_{2}}$ and $t_{3}=t_{\alpha_{1}+\alpha_{2}}$. For $\mathbf{y} \in \mathfrak{D}^{(1)}$, the vertices of the polytopes $\mathcal{P}_{m_{1}, m_{2}, \mathbf{y}}$ in (4.23) are given by

$$
\begin{aligned}
0, y_{2} & \text { for } \mathcal{P}_{0,0, \mathbf{y}}, \\
y_{2}, y_{1} & \text { for } \mathcal{P}_{0,1, \mathbf{y}}, \\
y_{1}, 1 & \text { for } \mathcal{P}_{1,1, \mathbf{y}} .
\end{aligned}
$$

Then by Theorem 4.5 and Lemma 2.9, we have

$$
\begin{aligned}
& F^{(1)}\left(\mathbf{t}, \mathbf{y} ; A_{2}\right) \\
&= \frac{t_{1} t_{2} t_{3} e^{t_{1} y_{1}+t_{2} y_{2}}}{\left(e^{t_{1}}-1\right)\left(e^{t_{2}}-1\right)\left(e^{t_{3}}-1\right)} \\
& \quad \times\left(y_{2} \frac{e^{\left(t_{3}-t_{1}-t_{2}\right) y_{2}}-1}{\left(t_{3}-t_{1}-t_{2}\right) y_{2}}+e^{t_{2}}\left(y_{1}-y_{2}\right) \frac{\left(e^{\left(t_{3}-t_{1}-t_{2}\right) y_{1}}-e^{\left(t_{3}-t_{1}-t_{2}\right) y_{2}}\right)}{\left(t_{3}-t_{1}-t_{2}\right)\left(y_{1}-y_{2}\right)}\right. \\
&\left.\quad+e^{t_{1}+t_{2}}\left(1-y_{1}\right) \frac{\left(e^{t_{3}-t_{1}-t_{2}}-e^{\left(t_{3}-t_{1}-t_{2}\right) y_{1}}\right)}{\left(t_{3}-t_{1}-t_{2}\right)\left(1-y_{1}\right)}\right)
\end{aligned}
$$

Hence by the Taylor expansion of (7.8), we have

$$
\begin{aligned}
B_{2,2,2}^{(1)}\left(\mathbf{y} ; A_{2}\right)= & \frac{1}{30240}+\frac{1}{360}\left(y_{1} y_{2}-y_{1}^{2}-y_{2}^{2}\right)+\frac{1}{144}\left(3 y_{1} y_{2}^{2}-3 y_{1}^{2} y_{2}+2 y_{1}^{3}\right) \\
& +\frac{1}{72}\left(-2 y_{1} y_{2}^{3}-3 y_{1}^{2} y_{2}^{2}+4 y_{1}^{3} y_{2}-2 y_{1}^{4}+y_{2}^{4}\right) \\
& +\frac{1}{240}\left(-5 y_{1} y_{2}^{4}+10 y_{1}^{2} y_{2}^{3}+10 y_{1}^{3} y_{2}^{2}-15 y_{1}^{4} y_{2}+6 y_{1}^{5}\right) \\
& +\frac{1}{240}\left(6 y_{1} y_{2}^{5}-5 y_{1}^{2} y_{2}^{4}-5 y_{1}^{4} y_{2}^{2}+6 y_{1}^{5} y_{2}-2 y_{1}^{6}-2 y_{2}^{6}\right) .
\end{aligned}
$$


Similarly we can calculate $B_{2,2,2}^{(2)}\left(\mathbf{y} ; A_{2}\right)$ for $\mathbf{y} \in \mathfrak{D}^{(2)}$, which coincides with (7.9) with $y_{1}$ and $y_{2}$ exchanged. In the case $\mathbf{y}=0$, from Lemma 3.4, we have

$$
\begin{aligned}
F\left(\mathbf{t} ; A_{2}\right)= & \frac{t_{1} t_{2} t_{3} e^{t_{1}+t_{2}}\left(e^{t_{3}-t_{1}-t_{2}}-1\right)}{\left(e^{t_{1}}-1\right)\left(e^{t_{2}}-1\right)\left(e^{t_{3}}-1\right)\left(t_{3}-t_{1}-t_{2}\right)} \\
=1 & +\frac{1}{12}\left(t_{1} t_{2}-t_{1} t_{3}-t_{2} t_{3}\right)+\frac{1}{360}\left(t_{1} t_{2} t_{3}^{2}-t_{1}^{2} t_{2} t_{3}-t_{1} t_{2}^{2} t_{3}\right) \\
& \quad+\frac{1}{720}\left(t_{1}^{2} t_{2}^{2}+t_{1}^{2} t_{3}^{2}+t_{2}^{2} t_{3}^{2}\right)+\frac{1}{30240} t_{1}^{2} t_{2}^{2} t_{3}^{2}+\cdots,
\end{aligned}
$$

by letting $\mathbf{y} \rightarrow 0$ in (7.8). Therefore by Theorem 4.6, we recover Mordell's formula [24]:

$$
\zeta_{2}\left(2,2,2 ; A_{2}\right)=(-1)^{3} \frac{(2 \pi \sqrt{-1})^{6}}{3 !} \frac{1}{30240}=\frac{\pi^{6}}{2835} .
$$

We also discuss the action of the extended Weyl group. Note that $\operatorname{Aut}(\Delta)$ is generated by $\left\{\sigma_{1}, \sigma_{2}, \omega\right\}$ where $\omega$ is a unique element of $\Omega$ such that $\omega \neq \mathrm{id}$, and hence $\omega \alpha_{1}=\alpha_{2}$, $\omega \lambda_{1}=\lambda_{2}$ and $\omega^{2}=$ id. Also note that $\alpha_{1}=2 \lambda_{1}-\lambda_{2}$ and $\alpha_{2}=2 \lambda_{2}-\lambda_{1}$. For $\mathbf{y} \in \mathfrak{D}^{(1)}$, we have

$$
\begin{aligned}
& \left\langle\alpha_{1}^{\vee}+\sigma_{1} \mathbf{y}, \lambda_{1}\right\rangle=1+\left\langle\mathbf{y}, \sigma_{1} \lambda_{1}\right\rangle=1+\left\langle\mathbf{y}, \lambda_{1}-\alpha_{1}\right\rangle=1-y_{1}+y_{2}, \\
& \left\langle\alpha_{1}^{\vee}+\sigma_{1} \mathbf{y}, \lambda_{2}\right\rangle=\left\langle\mathbf{y}, \sigma_{1} \lambda_{2}\right\rangle=\left\langle\mathbf{y}, \lambda_{2}\right\rangle=y_{2},
\end{aligned}
$$

which implies

$$
0<\left\langle\alpha_{1}^{\vee}+\sigma_{1} \mathbf{y}, \lambda_{2}\right\rangle<\left\langle\alpha_{1}^{\vee}+\sigma_{1} \mathbf{y}, \lambda_{1}\right\rangle<1 .
$$

Therefore we have $\tau\left(\alpha_{1}^{\vee}\right) \sigma_{1} \mathfrak{D}^{(1)}=\mathfrak{D}^{(1)}$ and in a similar way $\sigma_{1} \mathfrak{D}^{(2)}=\mathfrak{D}^{(2)}$, and so on. Thus from (6.69) we see that for $f=\left(f_{1}, f_{2}\right)$ with $f_{1}, f_{2} \in \mathbb{Q}[\mathbf{y}]$, the action of $\operatorname{Aut}(\Delta)$ is given by

$$
\begin{aligned}
\left(\phi\left(\sigma_{1}\right) f\right)(\mathbf{y}) & =\left(f_{1}\left(\alpha_{1}^{\vee}+\sigma_{1} \mathbf{y}\right), f_{2}\left(\sigma_{1} \mathbf{y}\right)\right), \\
\left(\phi\left(\sigma_{2}\right) f\right)(\mathbf{y}) & =\left(f_{1}\left(\sigma_{2} \mathbf{y}\right), f_{2}\left(\alpha_{2}^{\vee}+\sigma_{2} \mathbf{y}\right)\right), \\
(\phi(\omega) f)(\mathbf{y}) & =\left(f_{2}(\omega \mathbf{y}), f_{1}(\omega \mathbf{y})\right),
\end{aligned}
$$

or in terms of coordinates,

$$
\begin{aligned}
\left(\phi\left(\sigma_{1}\right) f\right)\left(y_{1}, y_{2}\right) & =\left(f_{1}\left(1-y_{1}+y_{2}, y_{2}\right), f_{2}\left(-y_{1}+y_{2}, y_{2}\right)\right), \\
\left(\phi\left(\sigma_{2}\right) f\right)\left(y_{1}, y_{2}\right) & =\left(f_{1}\left(y_{1}, y_{1}-y_{2}\right), f_{2}\left(y_{1}, 1+y_{1}-y_{2}\right)\right), \\
(\phi(\omega) f)\left(y_{1}, y_{2}\right) & =\left(f_{2}\left(y_{2}, y_{1}\right), f_{1}\left(y_{2}, y_{1}\right)\right) .
\end{aligned}
$$

Then for

$$
\begin{aligned}
& \mathbf{B}_{2,2,2}^{(1)}\left(\mathbf{y} ; A_{2}\right)=\left(B_{2,2,2}^{(1)}\left(\mathbf{y} ; A_{2}\right), 0\right), \\
& \mathbf{B}_{2,2,2}^{(2)}\left(\mathbf{y} ; A_{2}\right)=\left(0, B_{2,2,2}^{(2)}\left(\mathbf{y} ; A_{2}\right)\right),
\end{aligned}
$$

we have

$$
\begin{aligned}
\phi\left(\sigma_{1}\right) \mathbf{B}_{2,2,2}^{(1)} & =\mathbf{B}_{2,2,2}^{(1)}, \\
\phi\left(\sigma_{2}\right) \mathbf{B}_{2,2,2}^{(1)} & =\mathbf{B}_{2,2,2}^{(1)}, \\
\phi(\omega) \mathbf{B}_{2,2,2}^{(1)} & =\mathbf{B}_{2,2,2}^{(2)} .
\end{aligned}
$$


More generally we can show

$$
\begin{aligned}
\phi\left(\sigma_{1}\right) \mathbf{B}_{k_{1}, k_{2}, k_{3}}^{(1)} & =\mathbf{B}_{k_{1}, k_{3}, k_{2}}^{(1)}, \\
\phi\left(\sigma_{2}\right) \mathbf{B}_{k_{1}, k_{2}, k_{3}}^{(1)} & =\mathbf{B}_{k_{3}, k_{2}, k_{1}}^{(1)}, \\
\phi(\omega) \mathbf{B}_{k_{1}, k_{2}, k_{3}}^{(1)} & =\mathbf{B}_{k_{2}, k_{1}, k_{3}}^{(2)} .
\end{aligned}
$$

Example 7.3. The set of positive roots of type $C_{2}\left(\simeq B_{2}\right)$ consists of $\alpha_{1}, \alpha_{2}, 2 \alpha_{1}+\alpha_{2}$ and $\alpha_{1}+\alpha_{2}$. Let $t_{1}=t_{\alpha_{1}}, t_{2}=t_{\alpha_{2}}, t_{3}=t_{2 \alpha_{1}+\alpha_{2}}$ and $t_{4}=t_{\alpha_{1}+\alpha_{2}}$. The vertices $\left(x_{2 \alpha_{1}+\alpha_{2}}, x_{\alpha_{1}+\alpha_{2}}\right)$ of the polytopes $\mathcal{P}_{m_{1}, m_{2}}$ in (4.25) are given by

$$
\begin{array}{ll}
(0,0),(0,1 / 2),(1,0) & \text { for } \mathcal{P}_{1,1}, \\
(0,1),(0,1 / 2),(1,0) & \text { for } \mathcal{P}_{1,2}, \\
(0,1),(1,1 / 2),(1,0) & \text { for } \mathcal{P}_{2,2}, \\
(0,1),(1,1 / 2),(1,1) & \text { for } \mathcal{P}_{2,3} .
\end{array}
$$

Then by Lemmas 2.9 and 2.10 , we obtain

$$
\begin{aligned}
F\left(\mathbf{t} ; C_{2}\right)= & \left(\prod_{j=1}^{4} \frac{t_{j}}{e^{t_{j}}-1}\right) G\left(\mathbf{t} ; C_{2}\right) \\
=1 & +\frac{1}{2880}\left(2 t_{1} t_{2} t_{3}^{2}-4 t_{1} t_{2} t_{4}^{2}-2 t_{1} t_{2}^{2} t_{3}+4 t_{1} t_{2}^{2} t_{4}-4 t_{1} t_{3} t_{4}^{2}-4 t_{1} t_{3}^{2} t_{4}\right. \\
\quad & \left.\quad+t_{1}^{2} t_{2} t_{3}-4 t_{1}^{2} t_{2} t_{4}-4 t_{1}^{2} t_{3} t_{4}-t_{2} t_{3} t_{4}^{2}-2 t_{2} t_{3}^{2} t_{4}-2 t_{2}^{2} t_{3} t_{4}\right) \\
& +\frac{1}{241920}\left(3 t_{1} t_{2} t_{3}^{2} t_{4}^{2}-3 t_{1} t_{2}^{2} t_{3} t_{4}^{2}-3 t_{1}^{2} t_{2} t_{3}^{2} t_{4}-3 t_{1}^{2} t_{2}^{2} t_{3} t_{4}+2 t_{1}^{2} t_{2}^{2} t_{3}^{2}+8 t_{1}^{2} t_{2}^{2} t_{4}^{2}\right. \\
& \left.\quad+8 t_{1}^{2} t_{3}^{2} t_{4}^{2}+2 t_{2}^{2} t_{3}^{2} t_{4}^{2}\right) \\
& +\frac{1}{9676800} t_{1}^{2} t_{2}^{2} t_{3}^{2} t_{4}^{2}+\cdots,
\end{aligned}
$$

where

$$
\begin{gathered}
\frac{e^{t_{3}}}{\left(t_{1}+t_{2}-t_{3}\right)\left(t_{1}-2 t_{3}+t_{4}\right)}-\frac{2 e^{\frac{t_{1}}{2}+\frac{t_{4}}{2}}}{\left(t_{1}+2 t_{2}-t_{4}\right)\left(t_{1}-2 t_{3}+t_{4}\right)}+\frac{e^{t_{1}+t_{2}}}{\left(t_{1}+t_{2}-t_{3}\right)\left(t_{1}+2 t_{2}-t_{4}\right)} \\
-\frac{e^{t_{2}+t_{3}}}{\left(t_{2}+t_{3}-t_{4}\right)\left(t_{1}-2 t_{3}+t_{4}\right)}+\frac{e^{t_{4}}}{\left(t_{1}+2 t_{2}-t_{4}\right)\left(t_{2}+t_{3}-t_{4}\right)}+\frac{e^{\frac{t_{1}}{2}+t_{2}+\frac{t_{4}}{2}}}{\left(t_{1}+2 t_{2}-t_{4}\right)\left(t_{1}-2 t_{3}+t_{4}\right)} \\
+\frac{2 e^{\frac{t_{1}}{2}+t_{3}+\frac{t_{4}}{2}}}{\left(t_{1}+2 t_{2}-t_{4}\right)\left(t_{1}-2 t_{3}+t_{4}\right)}+\frac{e^{t_{1}+t_{2}+t_{3}}}{\left(t_{2}+t_{3}-t_{4}\right)\left(t_{1}+2 t_{2}-t_{4}\right)}-\frac{e^{t_{1}+t_{4}}}{\left(t_{2}+t_{3}-t_{4}\right)\left(t_{1}-2 t_{3}+t_{4}\right)} \\
+\frac{e^{t_{3}+t_{4}}}{\left(t_{1}+t_{2}-t_{3}\right)\left(t_{1}+2 t_{2}-t_{4}\right)}-\frac{e^{\frac{t_{1}}{2}+t_{2}+t_{3}+\frac{t_{4}}{2}}}{\left(t_{1}+2 t_{2}-t_{4}\right)\left(t_{1}-2 t_{3}+t_{4}\right)}+\frac{e^{t_{1}+t_{2}+t_{4}}}{\left(t_{1}+t_{2}-t_{3}\right)\left(t_{1}-2 t_{3}+t_{4}\right)} .
\end{gathered}
$$

Therefore

$$
\zeta_{2}\left(2,2,2,2 ; C_{2}\right)=(-1)^{4} \frac{(2 \pi \sqrt{-1})^{8}}{2 ! \cdot 2^{2}} \frac{1}{9676800}=\frac{\pi^{8}}{302400}
$$


and so

$$
\zeta_{W}\left(2 ; C_{2}\right)=6^{2} \frac{\pi^{8}}{302400}=\frac{\pi^{8}}{8400} .
$$

Example 7.4. The set of positive roots of type $A_{3}$ consists of $\alpha_{1}, \alpha_{2}, \alpha_{3}, \alpha_{1}+\alpha_{2}, \alpha_{2}+\alpha_{3}$ and $\alpha_{1}+\alpha_{2}+\alpha_{3}$. Let $t_{1}=t_{\alpha_{1}}, t_{2}=t_{\alpha_{2}}, t_{3}=t_{\alpha_{3}}, t_{4}=t_{\alpha_{1}+\alpha_{2}}, t_{5}=t_{\alpha_{2}+\alpha_{3}}$ and $t_{6}=t_{\alpha_{1}+\alpha_{2}+\alpha_{3}}$. The vertices $\left(x_{\alpha_{1}+\alpha_{2}}, x_{\alpha_{2}+\alpha_{3}}, x_{\alpha_{1}+\alpha_{2}+\alpha_{3}}\right)$ of the polytopes $\mathcal{P}_{m_{1}, m_{2}, m_{3}}$ in (4.25) are given by

$$
\begin{array}{ll}
(0,0,0),(0,0,1),(0,1,0),(1,0,0) & \text { for } \mathcal{P}_{1,1,1}, \\
(0,0,1),(0,1,0),(1,0,0),(1,1,0) & \text { for } \mathcal{P}_{1,2,1}, \\
(0,0,1),(0,1,0),(0,1,1),(1,1,0) & \text { for } \mathcal{P}_{1,2,2}, \\
(0,0,1),(1,0,0),(1,0,1),(1,1,0) & \text { for } \mathcal{P}_{2,2,1}, \\
(0,0,1),(0,1,1),(1,0,1),(1,1,0) & \text { for } \mathcal{P}_{2,2,2}, \\
(0,1,1),(1,0,1),(1,1,0),(1,1,1) & \text { for } \mathcal{P}_{2,3,2}
\end{array}
$$

Then by Lemmas 2.9 and 2.10, we obtain

$$
\begin{aligned}
F\left(\mathbf{t} ; A_{3}\right) & =\left(\prod_{j=1}^{6} \frac{t_{j}}{e^{t_{j}}-1}\right) G\left(\mathbf{t} ; A_{3}\right) \\
& =1+\cdots+\frac{23}{435891456000} t_{1}^{2} t_{2}^{2} t_{3}^{2} t_{4}^{2} t_{5}^{2} t_{6}^{2}+\cdots,
\end{aligned}
$$


where

$$
\begin{aligned}
& \text { (7.27) } G\left(\mathbf{t} ; A_{3}\right)= \\
& \frac{e^{t_{3}+t_{4}}}{\left(t_{1}+t_{2}-t_{4}\right)\left(t_{1}-t_{3}-t_{4}+t_{5}\right)\left(t_{3}+t_{4}-t_{6}\right)}-\frac{e^{t_{1}+t_{5}}}{\left(t_{2}+t_{3}-t_{5}\right)\left(t_{1}-t_{3}-t_{4}+t_{5}\right)\left(t_{1}+t_{5}-t_{6}\right)} \\
& -\frac{e^{t_{6}}}{\left(t_{1}+t_{2}+t_{3}-t_{6}\right)\left(t_{3}+t_{4}-t_{6}\right)\left(t_{1}+t_{5}-t_{6}\right)}+\frac{e^{t_{1}+t_{2}+t_{3}}}{\left(t_{1}+t_{2}-t_{4}\right)\left(t_{2}+t_{3}-t_{5}\right)\left(t_{1}+t_{2}+t_{3}-t_{6}\right)} \\
& -\frac{e^{t_{4}+t_{5}}}{\left(t_{1}+t_{2}-t_{4}\right)\left(t_{2}+t_{3}-t_{5}\right)\left(t_{2}-t_{4}-t_{5}+t_{6}\right)}-\frac{e^{t_{2}+t_{3}+t_{4}}}{\left(t_{2}+t_{3}-t_{5}\right)\left(t_{1}-t_{3}-t_{4}+t_{5}\right)\left(t_{3}+t_{4}-t_{6}\right)} \\
& +\frac{e^{t_{1}+t_{2}+t_{5}}}{\left(t_{1}+t_{2}-t_{4}\right)\left(t_{1}-t_{3}-t_{4}+t_{5}\right)\left(t_{1}+t_{5}-t_{6}\right)}+\frac{e^{t_{2}+t_{6}}}{\left(t_{3}+t_{4}-t_{6}\right)\left(t_{1}+t_{5}-t_{6}\right)\left(t_{2}-t_{4}-t_{5}+t_{6}\right)} \\
& +\frac{e^{t_{3}+t_{4}+t_{5}}}{\left(t_{1}+t_{2}-t_{4}\right)\left(t_{3}+t_{4}-t_{6}\right)\left(t_{2}-t_{4}-t_{5}+t_{6}\right)}-\frac{e^{t_{5}+t_{6}}}{\left(t_{2}+t_{3}-t_{5}\right)\left(t_{1}+t_{2}+t_{3}-t_{6}\right)\left(t_{3}+t_{4}-t_{6}\right)} \\
& +\frac{e^{t_{1}+t_{2}+t_{3}+t_{5}}}{\left(t_{1}+t_{2}-t_{4}\right)\left(t_{1}+t_{2}+t_{3}-t_{6}\right)\left(t_{1}+t_{5}-t_{6}\right)}-\frac{e^{t_{2}+t_{3}+t_{6}}}{\left(t_{2}+t_{3}-t_{5}\right)\left(t_{1}+t_{5}-t_{6}\right)\left(t_{2}-t_{4}-t_{5}+t_{6}\right)} \\
& +\frac{e^{t_{1}+t_{4}+t_{5}}}{\left(t_{2}+t_{3}-t_{5}\right)\left(t_{1}+t_{5}-t_{6}\right)\left(t_{2}-t_{4}-t_{5}+t_{6}\right)}-\frac{e^{t_{4}+t_{6}}}{\left(t_{1}+t_{2}-t_{4}\right)\left(t_{1}+t_{2}+t_{3}-t_{6}\right)\left(t_{1}+t_{5}-t_{6}\right)} \\
& +\frac{e^{t_{1}+t_{2}+t_{3}+t_{4}}}{\left(t_{2}+t_{3}-t_{5}\right)\left(t_{1}+t_{2}+t_{3}-t_{6}\right)\left(t_{3}+t_{4}-t_{6}\right)}-\frac{e^{t_{1}+t_{2}+t_{6}}}{\left(t_{1}+t_{2}-t_{4}\right)\left(t_{3}+t_{4}-t_{6}\right)\left(t_{2}-t_{4}-t_{5}+t_{6}\right)} \\
& -\frac{e^{t_{1}+t_{3}+t_{4}+t_{5}}}{\left(t_{1}+t_{5}-t_{6}\right)\left(t_{2}-t_{4}-t_{5}+t_{6}\right)\left(t_{3}+t_{4}-t_{6}\right)}-\frac{e^{t_{3}+t_{4}+t_{6}}}{\left(t_{1}+t_{2}-t_{4}\right)\left(t_{1}-t_{3}-t_{4}+t_{5}\right)\left(t_{1}+t_{5}-t_{6}\right)} \\
& +\frac{e^{t_{1}+t_{5}+t_{6}}}{\left(t_{2}+t_{3}-t_{5}\right)\left(t_{1}-t_{3}-t_{4}+t_{5}\right)\left(t_{3}+t_{4}-t_{6}\right)}+\frac{e^{t_{1}+t_{2}+t_{3}+t_{6}}}{\left(t_{1}+t_{2}-t_{4}\right)\left(t_{2}+t_{3}-t_{5}\right)\left(t_{2}-t_{4}-t_{5}+t_{6}\right)} \\
& -\frac{e^{t_{4}+t_{5}+t_{6}}}{\left(t_{1}+t_{2}-t_{4}\right)\left(t_{2}+t_{3}-t_{5}\right)\left(t_{1}+t_{2}+t_{3}-t_{6}\right)}+\frac{e^{t_{1}+t_{2}+t_{3}+t_{4}+t_{5}}}{\left(t_{1}+t_{2}+t_{3}-t_{6}\right)\left(t_{3}+t_{4}-t_{6}\right)\left(t_{1}+t_{5}-t_{6}\right)} \\
& +\frac{e^{t_{2}+t_{3}+t_{4}+t_{6}}}{\left(t_{2}+t_{3}-t_{5}\right)\left(t_{1}-t_{3}-t_{4}+t_{5}\right)\left(t_{1}+t_{5}-t_{6}\right)}-\frac{e^{t_{1}+t_{2}+t_{5}+t_{6}}}{\left(t_{1}+t_{2}-t_{4}\right)\left(t_{1}-t_{3}-t_{4}+t_{5}\right)\left(t_{3}+t_{4}-t_{6}\right)} .
\end{aligned}
$$

Therefore we obtain

$$
\zeta_{3}\left(2,2,2,2,2,2 ; A_{3}\right)=(-1)^{6} \frac{(2 \pi \sqrt{-1})^{12}}{4 !} \frac{23}{435891456000}=\frac{23}{2554051500} \pi^{12},
$$

which implies a formula of Gunnells-Sczech [4]:

$$
\zeta_{W}\left(2 ; A_{3}\right)=12^{2} \frac{23}{2554051500} \pi^{12}=\frac{92}{70945875} \pi^{12} .
$$

In higher rank root systems, generating functions are more involved, since the polytopes are not simplicial any longer. For instance, we have the generating function of type $G_{2}, A_{4}$, $B_{3}$ and $C_{3}$ with 1010 terms, 5040 terms, 19908 terms and 20916 terms respectively by use of triangulation. In [14], we will improve Theorem 4.5] and will give more compact forms of the generating functions $F(\mathbf{t}, \mathbf{y} ; \Delta)$, which do not depend on simplicial decompositions. As a result, the numbers of terms in the above generating functions reduce to $15,125,68$ and 68 respectively (as for the $G_{2}$ case, see [13]).

Example 7.5. In Theorem 4.3, we have already given general forms of functional relations among zeta-functions of root systems. In previous examples we observed generating 
functions and special values in several cases, but here, we treat examples of explicit functional relations which can be deduced from the general forms. First consider the $A_{2}$ case (see Example 7.2). Set

$$
\Delta_{+}=\Delta_{+}\left(A_{2}\right)=\left\{\alpha_{1}, \alpha_{2}, \alpha_{1}+\alpha_{2}\right\},
$$

and $\mathbf{y}=0, \mathbf{s}=(2, s, 2)$ for $s \in \mathbb{C}$ with $\Re s>1, I=\{2\}$, that is, $\Delta_{I+}=\left\{\alpha_{2}\right\}$. Then, from (4.6), we can write the left-hand side of (4.11) as

$$
\begin{aligned}
S(\mathbf{s}, \mathbf{y} ; I ; \Delta) & =\sum_{m, n=1}^{\infty} \frac{1}{m^{2} n^{s}(m+n)^{2}}+\sum_{\substack{m, n=1 \\
m \neq n}}^{\infty} \frac{1}{m^{2} n^{s}(-m+n)^{2}} \\
& =2 \zeta_{2}\left(2, s, 2 ; A_{2}\right)+\zeta_{2}\left(2,2, s ; A_{2}\right) .
\end{aligned}
$$

On the other hand, the right-hand side of (4.11) is

$$
\begin{aligned}
& \left(\frac{(2 \pi \sqrt{-1})^{2}}{2 !}\right)^{2} \sum_{m=1}^{\infty} \frac{1}{m^{s}} \int_{0}^{1} e^{-2 \pi \sqrt{-1} m x} L_{2}(x, 0) L_{2}(-x, 0) d x \\
& =\left(\frac{(2 \pi \sqrt{-1})^{2}}{2 !}\right)^{2} \sum_{m=1}^{\infty} \frac{1}{m^{s}} \int_{0}^{1} e^{-2 \pi \sqrt{-1} m x} B_{2}(x) B_{2}(1-x) d x
\end{aligned}
$$

by using (4.17). From well-known properties of Bernoulli polynomials, we can calculate the above integral (for details, see Nakamura [25]) and can recover from (4.11) the formula

$$
2 \zeta_{2}\left(2, s, 2 ; A_{2}\right)+\zeta_{2}\left(2,2, s ; A_{2}\right)=4 \zeta(2) \zeta(s+2)-6 \zeta(s+4),
$$

proved in [33] (see also [25]). The function $\zeta_{2}\left(\mathbf{s} ; A_{2}\right)$ can be continued meromorphically to the whole space $\mathbb{C}^{3}([17])$, so (7.30) holds for any $s \in \mathbb{C}$ except for singularities on the both sides. In particular when $s=2$, we obtain (7.11). Similarly we can treat the $C_{2}\left(\simeq B_{2}\right)$ case and give some functional relations from (4.11) by combining the meromorphic continuation of $\zeta_{2}\left(\mathbf{s} ; C_{2}\right)$ which has been shown in [18].

\section{REFERENCES}

[1] T. M. Apostol, Introduction to Analytic Number Theory, Springer, 1976.

[2] N. Bourbaki, Groupes et Algèbres de Lie, Chapitres 4, 5 et 6, Hermann, Paris, 1968.

[3] D. M. Bradley, Partition identities for the multiple zeta function, in 'Zeta Functions, Topology and Quantum Physics', Developments in Mathematics 14, T. Aoki et al. (eds.), Springer, New York, 2005, pp. 19-29.

[4] P. E. Gunnells and R. Sczech, Evaluation of Dedekind sums, Eisenstein cocycles, and special values of $L$-functions, Duke Math. J. 118 (2003), 229-260.

[5] T. Hibi, Algebraic combinatorics on convex polytopes, Carslaw Publications, Australia, 1992.

[6] J. E. Humphreys, Introduction to Lie algebras and representation theory, Graduate Texts in Mathematics, Vol. 9, Springer-Verlag, New York-Berlin, 1972.

[7] J. E. Humphreys, Reflection groups and Coxeter groups, Cambridge University Press, Cambridge, 1990.

[8] V. G. Kac, Infinite-dimensional Lie algebras, 3rd ed., Cambridge University Press, Cambridge, 1990.

[9] Y. Komori, K. Matsumoto and H. Tsumura, Zeta-functions of root systems, in 'Proceedings of the Conference on L-functions' (Fukuoka, 2006), L. Weng and M. Kaneko (eds), World Scientific, 2007, pp. 115-140.

[10] Y. Komori, K. Matsumoto and H. Tsumura, Zeta and $L$-functions and Bernoulli polynomials of root systems, Proc. Japan Acad., Series A, 84 (2008), 57-62.

[11] Y. Komori, K. Matsumoto and H. Tsumura, Zeta-functions of root systems, their functional relations, and Dynkin diagrams, in 'Analytic Number Theory' (Kyoto, 2006), RIMS Kokyuroku, to appear.

[12] Y. Komori, K. Matsumoto and H. Tsumura, On Witten multiple zeta-functions associated with semisimple Lie algebras II, J. Math. Soc. Japan, to appear. 
[13] Y. Komori, K. Matsumoto and H. Tsumura, On Witten multiple zeta-functions associated with semisimple Lie algebras IV, preprint, arXiv:0907.0972.

[14] Y. Komori, K. Matsumoto and H. Tsumura, On multiple Bernoulli polynomials and multiple $L$ functions of root systems, Proc. London Math. Soc., to appear.

[15] Y. Komori, K. Matsumoto and H. Tsumura, Functional relations for zeta-functions of root systems, preprint.

[16] I. G. Macdonald, Symmetric functions and Hall polynomials, 2nd ed., Oxford Univ. Press, Oxford, 1995.

[17] K. Matsumoto, On the analytic continuation of various multiple zeta-functions, in 'Number Theory for the Millennium II, Proc. Millennial Conference on Number Theory', M. A. Bennett et al. (eds.), A K Peters, 2002, pp. 417-440.

[18] K. Matsumoto, On Mordell-Tornheim and other multiple zeta-functions, in 'Proceedings of the Session in analytic number theory and Diophantine equations' (Bonn, January-June 2002), D. R. HeathBrown and B. Z. Moroz (eds.), Bonner Mathematische Schriften Nr. 360, Bonn 2003, n.25, 17pp.

[19] K. Matsumoto, T. Nakamura, H. Ochiai and H. Tsumura, On value-relations, functional relations and singularities of Mordell-Tornheim and related triple zeta-functions, Acta Arith. 132 (2008), 99-125.

[20] K. Matsumoto, T. Nakamura and H. Tsumura, Functional relations and special values of MordellTornheim triple zeta and $L$-functions, Proc. Amer. Math. Soc. 136 (2008), 2135-2145.

[21] K. Matsumoto and H. Tsumura, On Witten multiple zeta-functions associated with semisimple Lie algebras I, Ann. Inst. Fourier, 56 (2006), 1457-1504.

[22] K. Matsumoto and H. Tsumura, Functional relations for various multiple zeta-functions, in 'Analytic Number Theory' (Kyoto, 2005), RIMS Kokyuroku No. 1512 (2006), 179-190.

[23] K. Matsumoto and H. Tsumura, Functional relations among certain double polylogarithms and their character analogues, Šiauliai Math. Sem. 3 (11) (2008), 189-205.

[24] L. J. Mordell, On the evaluation of some multiple series, J. London Math. Soc. 33 (1958), 368-371.

[25] T. Nakamura, A functional relation for the Tornheim double zeta function, Acta Arith. 125 (2006), 257-263.

[26] M. V. Subbarao and R. Sitaramachandrarao, On some infinite series of L. J. Mordell and their analogues, Pacific J. Math. 119 (1985), 245-255.

[27] R. P. Stanley, Decompositions of rational convex polytopes, in 'Combinatorial mathematics, optimal designs and their applications' (Proc. Sympos. Combin. Math. and Optimal Design, Colorado State Univ., Fort Collins, Colo., 1978), Ann. Discrete Math. 6 (1980), 333-342.

[28] A. Szenes, Iterated residues and multiple Bernoulli polynomials, Internat. Math. Res. Notices, 18 (1998), 937-958.

[29] A. Szenes, Residue formula for rational trigonometric sums, Duke Math. J. 118 (2003) 189-228.

[30] L. Tornheim, Harmonic double series, Amer. J. Math. 72 (1950), 303-314.

[31] H. Tsumura, On Witten's type of zeta values attached to SO(5), Arch. Math. (Basel) 84 (2004), $147-152$.

[32] H. Tsumura, On some functional relations between Mordell-Tornheim double $L$-functions and Dirichlet $L$-functions, J. Number Theory, 120 (2006), 161-178.

[33] H. Tsumura, On functional relations between the Mordell-Tornheim double zeta functions and the Riemann zeta function, Math. Proc. Cambridge Philos. Soc., 142 (2007), 395-405.

[34] E. T. Whittaker and G. N. Watson, A Course of Modern Analysis, 4th ed., Cambridge University Press (1958).

[35] E. Witten, On quantum gauge theories in two dimensions, Comm. Math. Phys. 141 (1991), 153-209.

[36] D. Zagier, Values of zeta functions and their applications, in 'First European Congress of Mathematics' Vol. II, A. Joseph et al. (eds.), Progr. Math. 120, Birkhäuser, 1994, pp. 497-512.

[37] G. M. Ziegler, Lectures on polytopes, Springer-Verlag, New York, 1995. 